

\title{
A Theory of Photometric Stereo for a General Class of Reflectance Maps.
}

\author{
Hemant D. Tagare
}

\begin{abstract}
Photometric stereo is an image processing technique for $2 \frac{1}{2}$ dimensional surface reconstruction from local shading. The classical theory of photometric stereo has been developed only for surfaces that reflect in a Lambertian plus specular manner. However, there is plenty of experimental evidence that most real-world surfaces are not Lambertian plus specular.

This thesis develops the theory of photometric stereo for non-Lambertian surfaces. First, based on the physics of reflection and scattering, a general class of reflectance maps is proposed. This class is shown to model real world data more accurately than the Lambertian model. Then, the normalized photometric stereo equation using these reflectance maps is analyzed and conditions for a globally unique solution for the equation are obtained. Furthermore, the un-normalized photometric stereo equation is studied and conditions for getting a globally unique solution using only three light sources are identified. The problem of jointly estimating the reflectance map and the surface normal is proposed and shown to be ill-posed. A regularized solution to the problem is demonstrated. Finally, it is shown that extra light sources are needed to obtain a complete reconstruction of the surface, and the number of new light sources needed to achieve this is identified.
\end{abstract}




\section{Acknowledgments}

To begin with, I would like to thank my advisor Prof. Rui J. P. deFigueiredo for his guidance, help, and support during this work. I have greatly enjoyed working and discussing various aspects of this thesis with him.

Thanks are also due to my Ph.D. committee for working closely with me and providing appropriate feedback and help during various stages of my work.

I also gratefully acknowledge the faith and strength of my parents in supporting my decision to come back to graduate school.

Thanks are due to Dr. Guanron Chen for the many helpful discussions. Richard Murphy and Dr. J. D. Wise provided the necessary insight and help with software, UNIX and $\mathrm{C}$ without which the software for this thesis could have taken much longer to write and debug. Steven Kim patiently exposed rolls of film for me so that I could get the photographs to my satisfaction. Thanks are also due to all my friends in the Indian graduate student community for the moral support and all the late night dinners.

Finally, I must express deep gratitude and thanks to Lisa Berlinger for her love, sloppy joe's, proof reading, and, in times of stress, the ability to prevent my mind from jumping out from within the confines of my cranium. 


\section{Contents}

Abstract $\quad$ ii

Acknowledgments $\quad$ iii

List of Illustrations vi vi

1 Introduction $\quad 1$

$1.12 \frac{1}{2}$ Dimensional Surface Reconstruction . . . . . . . . . . 1

1.2 Shading and Photometric Stereo . . . . . . . . . . . . 2

1.3 The Scope of this Research . . . . . . . . . . . . 5

$\begin{array}{llr}2 \text { Background } & 7\end{array}$

2.1 Introduction . . . . . . . . . . . . . . . 7

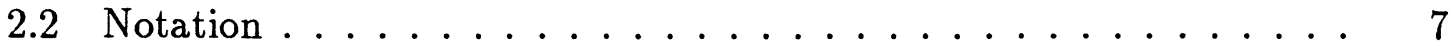

2.3 The Reflectance Map . . . . . . . . . . . . . 8

2.4 The Photometric Stereo Equations . . . . . . . . . . . . 13

2.5 Extended Light Sources ................. 15

3 Reflection from Solids $\quad 18$

3.1 Introduction . . . . . . . . . . . . . . 18

3.2 Scattering Lobes and Rough Surfaces. . . . . . . . . . . . . . . 19

3.3 The Basic Theories of Scattering and Specular Reflection . . . . . . 22

3.4 Inhomogeneous Solids . . . . . . . . . . . . . . 23

3.5 The Forescatter Lobe . . . . . . . . . . . . . . . . . . 26

3.6 The Normal Lobe . . . . . . . . . . . . . . . . . . . 33

3.7 The Backscatter Lobe . . . . . . . . . . . . . . 38

3.8 The Reflectance Map . . . . . . . . . . . . . . . . 40

3.9 A General Model for Reflectance Maps . . . . . . . . . . . . . 48

3.10 Experimental Evidence . . . . . . . . . . . . . . . . 49

4 The Normalized Photometric Stereo Equation 53 
4.1 Introduction . . . . . . . . . . . . . . . 53

4.2 Inversion of three-lobed maps $\ldots \ldots \ldots \ldots \ldots$

4.3 Three-lobed Isotropic Reflectance maps . . . . . . . . . . . . . . 62

4.4 Inversion of the $\mathrm{m}$-lobed $\operatorname{map} \ldots \ldots \ldots \ldots$

4.5 Experimental Results. . . . . . . . . . . . . . 6 66

5 The Un-normalized Photometric Stereo Equation $\quad 79$

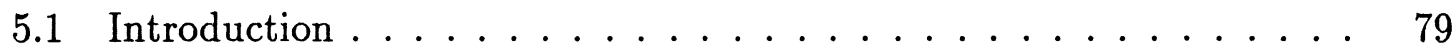

5.2 The manifold structure . . . . . . . . . . . . . . . . 79

5.3 The geometry of the manifold $S \ldots \ldots \ldots \ldots$

5.4 Single-lobed $\operatorname{map} \ldots \ldots \ldots \ldots \ldots . \ldots \ldots$

6 Joint estimation of surface normals and reflectance map 92

6.1 Introduction . . . . . . . . . . . . . . . . . . 92

6.2 An Approximate Solution . . . . . . . . . . . . . . . . 93

6.3 Ill-posed Nature of the Joint Estimate . . . . . . . . . . . . . 98

6.4 Regularization of Joint Estimation _ . . . . . . . . . . . 102

6.5 Experimental Results . . . . . . . . . . . . . 106

7 Completeness of Photometric Inversion 111

7.1 Introduction . . . . . . . . . . . . . . . . . 111

7.2 Convex Surfaces . . . . . . . . . . . . . . . . . . . . . . . 112

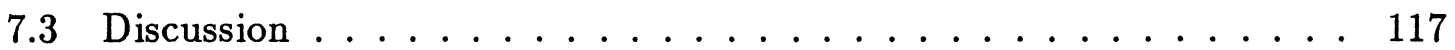

8 Conclusions $\quad 118$

A Surface Normal to Depth Conversion. 120

B Apparatus and Experimental Procedure 121

$\begin{array}{ll}\text { Bibliography } & 124\end{array}$ 


\section{Illustrations}

$1.12 \frac{1}{2}$ Dimensional representation . . . . . . . . . . . . . . . 2

1.2 Photometric stereo . . . . . . . . . . . . . . 3

1.3 Shading is a source of relative depth information . . . . . . . . . 3

2.1 The bi-directional reflectance distribution function . . . . . . . . 9

2.2 Illustration for Horn's relation . . . . . . . . . . . . . . . . 12

2.3 Extended light source . . . . . . . . . . . . . 16

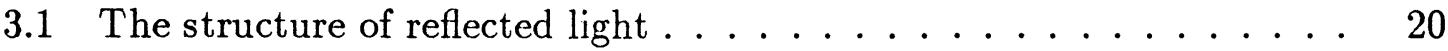

3.2 BRDF showing three diffuse lobes . . . . . . . . . . . . . 21

3.3 Height and slope distributions. Adapted from [58]. . . . . . . . . 25

3.4 Normalized BRDF $\frac{f_{r}\left(\theta_{r}, \pi ; \theta_{i}\right)}{f_{r}\left(\theta_{i}, \pi, \theta_{i}\right)}$ for a rough surface. Adapted from [56]. . 27

3.5 Origin of the forescatter lobe . . . . . . . . . . . . . 29

3.6 Results of the radiative transfer theory . . . . . . . . . . 36

3.7 BRDF showing strong backscatter. Adapted from [11] . . . . . . . . 39

3.8 Bi-static shadowing as a function of $\theta_{n} \ldots \ldots \ldots 42$

3.9 Comparison of forescatter reflectance map with and without shadowing 43

3.10 Comparison of the exact and approximate forescatter reflectance maps 44

3.11 Contours of the error in the approximation . . . . . . . . . 46

3.12 The backscatter reflectance map . . . . . . . . . . . . . . . 47

3.13 Principle directions for the m-lobed map . . . . . . . . . . 50

3.14 A spherical surface . . . . . . . . . . . . . . . . 51

3.15 Intensity in the principle plane . . . . . . . . . . 52

4.1 Geometry for defining the sets $C_{j} \ldots \ldots \ldots \ldots \ldots \ldots$

4.2 Principal Directions and Light Placement for Three Lobed map. . . . 63

4.3 Two spherical surfaces. . . . . . . . . . . . . . . . . . 67

4.4 Intensity in the principal plane for the Lambertian sphere. . . . . . . 68 
4.5 Reconstruction of the non-Lambertian surface using the Lambertian model.

4.6 Reconstruction of the non-Lambertian surface using the non-Lambertian model. . . . . . . . . . . . . . . . . 70

4.7 Depth profiles of reconstruction. . . . . . . . . . . . 71

4.8 Reconstruction of a Lambertian surface using a Lambertian model. . 72

4.9 Error in the surface normal reconstruction. . . . . . . . . . . 74

4.10 Image of a cylinder. . . . . . . . . . . . . . . . . . 75

4.11 Image of a spoon. . . . . . . . . . . . . . 75

4.12 Reconstruction of the cylinder. . . . . . . . . . . . . 76

4.13 Reconstruction of the spoon. . . . . . . . . . . . . . . 77

5.1 The shape of the manifold $S \ldots \ldots \ldots \ldots \ldots \ldots \ldots$

5.2 The intersection of $S$ and $\Gamma \ldots \ldots \ldots \ldots \ldots$

5.3 The function $J$ for the physical reflectance map . . . . . . . . . 86

6.1 Amplitude spectrum of $g(\cos \phi)$ for different $c \ldots \ldots 96$

6.2 Eight lights for 4 different $x_{i} \ldots \ldots \ldots \ldots \ldots$

6.3 Linear approximation to the forescatter lobe function. . . . . . . . . 100

6.4 Ill-posed nature of the joint estimate. . . . . . . . . . . . . . 103

6.5 Regularized reconstruction of the sphere. . . . . . . . . . 105

6.6 Histogram for $a_{1}^{*} \ldots \ldots \ldots \ldots$. . . . . . . . . . 107

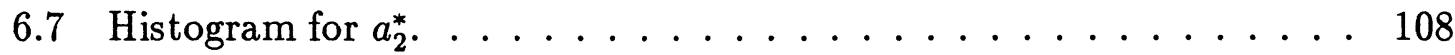

6.8 Regularized reconstruction of the cylinder. . . . . . . . . . . . 109

6.9 Regularized reconstruction of the spoon. . . . . . . . . . 110

7.1 2 Light sources do not yield a complete reconstruction. . . . . . . . 112

7.2 Configuration for Complete Reconstruction. . . . . . . . . . . 115

B.1 The Experimental Apparatus. . . . . . . . . . . . . . . 122 


\section{Chapter 1}

\section{Introduction}

"Now I say that whenever I conceive any material or corporeal substance, I immediately feel the need to think of it as bounded, and as having this or that shape; ..... From these conditions I cannot separate such a substance by any stretch of my imagination." - Gallileo Galilei. Letter to Don Virginio Cesarini.

\section{$1.12 \frac{1}{2}$ Dimensional Surface Reconstruction}

Humans and robots need a three-dimensional representation of objects of the external world in order to grasp and manipulate them effectively. For this purpose the visible bounding surface of a solid object provides a natural representation. Vision yields the most easily accessible sensory data that has information about the object surface encoded in it. Consequently, algorithms that reconstruct object surfaces in three dimensions from the observed scene have been the object of research for a long time [7].

Three-dimensional information is classically obtained from stereo vision [22]. However, the stereo vision problem is complex in its full generality and is the subject of much on going research [3]. Consequently, simpler versions of the three-dimensional vision problem have been formulated. In assembly line operations, for example, reconstructing surfaces in three-dimensions is often simplified to estimating the surface normal at all points on the surface. This is shown in figure 1.1. Estimating the surface normals yields what Marr calls a $2 \frac{1}{2}$ dimensional surface representation ${ }^{1}$ [38]. If we assume that the surface being imaged is continuous with a well defined surface normal everywhere, then from the estimate of the surface normal the relative depth $z(x, y)$ of the surface can be computed. The knowledge of the relative depth along with the knowledge of the location of the base line (the assembly line in fig. 1.1) yields a description of the surface in three dimensions.

\footnotetext{
${ }^{1}$ This term underlines that fact that surface normals yield knowledge which is more than twodimensional but less than full three-dimensional.
} 


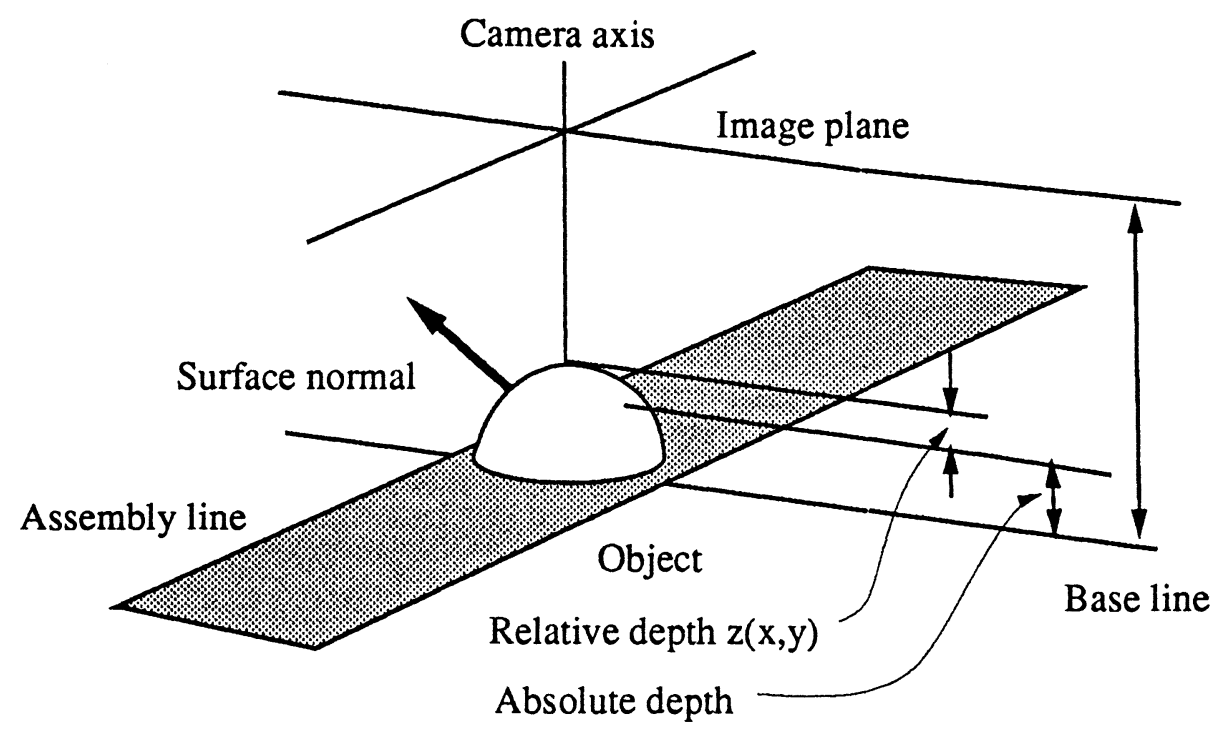

Figure 1.1 $2 \frac{1}{2}$ Dimensional representation

\subsection{Shading and Photometric Stereo}

In this work, we will investigate a method called the Photometric Stereo Method for $2 \frac{1}{2}$ dimensional surface reconstruction. Photometric stereo uses a single camera and a number of simple light sources. As depicted in figure 1.2, the scene is illuminated by one light source at a time and the resulting images are used to reconstruct the surface normal at every point.

Photometric stereo is based on a paradigm that is illustrated in Figure 1.3, where an image of a sphere illuminated by a single point light is shown. Note that the variation in the intensity from the brightest spot on the surface of the sphere to any of its occluding boundaries provides a strong shape (relative depth) clue. The variation in the intensity value due to change in surface normal is called shading. Photometric stereo estimates surface normals from local shading. The relation between the surface normal and the image intensity at any point in the image is specified by the Reflectance Map of the surface. Loosely speaking, a reflectance map is the non-linear transfer function from the surface normal to the image intensity. The form of the reflectance map depends on the nature of its two components: the manner in which the underlying surface reflects light and the manner in which this reflected light is 


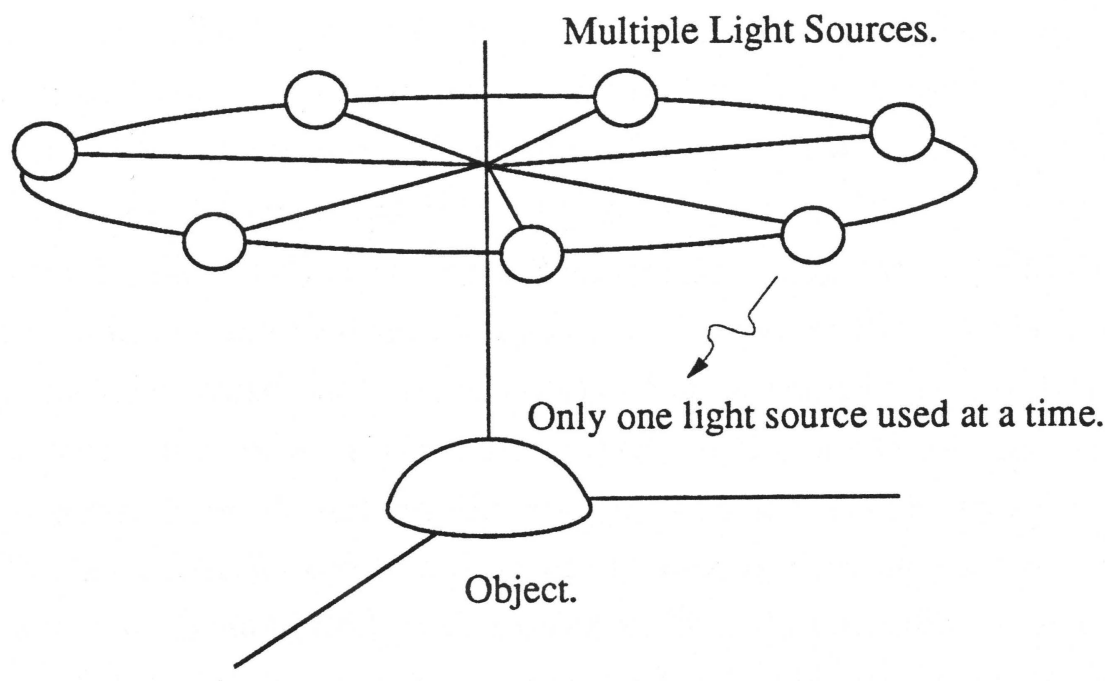

Figure 1.2 Photometric stereo

Figure 1.3 Shading is a source of relative depth information 
converted into an intensity value by the camera. Section 2.3 makes this relation explicit.

Photometric stereo was developed by Woodham [60] who reconstructed constant albedo Lambertian surfaces (see section 2.3 for the definitions of these terms). Woodham proved that three light sources are necessary and sufficient to get a globally unique solution to the the normalized photometric stereo equation 2.8 in the case of a Lambertian surface. Coleman and Jain [15] and Ray et al. [42] extended the analysis to variable albedo Lambertian surfaces. Silver [47] used experimentally measured reflectance maps in the normalized photometric stereo equation 2.8 (see section 2.4.) Interestingly, he discovered that for the class of surfaces he was investigating, three light sources were sufficient for a globally unique solution for the surface normal. This was an experimental result and he did not provide any theoretical justification for it. Ikeuchi [27] used distributed light sources to reconstruct purely specular surfaces. Nayar, Kanade and Ikeuchi [39] have extended Ikeuchi's results to reconstruct surfaces whose reflectance map can be modeled as a linear combination of a specular and Lambertian term. Recently, there have been some attempts [59] to use polarized light for photometric stereo.

Note that all of the work mentioned above, with the exception of Silver's thesis, assumes that the diffuse part of the reflectance map is Lambertian. The reason for the popularity of the specular-Lambertian model is the belief that reflection from a surface goes from being specular to Lambertian as the surface becomes rougher.

Contrary to this belief, there is considerable evidence that real world surfaces do not go from being highly specular to being Lambertian as they get rougher [8][52][55] [54][53]. As we shall demonstrate in chapter 3, diffuse reflection from most surfaces is caused by more than one physical mechanism. Only one of these mechanisms yields a Lambertian reflectance map. The others do not. Further, as shown in chapter 4, using the Lambertian model yields poor results on non-Lambertian surfaces. The main motivation for this research is the inclusion of non-Lambertian nature of reflection in photometric stereo theory.

Horn [21] and Silver [47] have attempted to set up non-Lambertian reflectance maps. However, their suggestions are heuristic in nature and are not based on any physical considerations. Also, there has not been any serious attempt to use these reflectance maps in photometric stereo. 


\subsection{The Scope of this Research}

Once the non-Lambertian nature of diffuse reflection is fully acknowledged, the need for a theory of non-Lambertian photometric stereo becomes apparent. In this research we will restrict ourselves to diffusely reflecting surfaces and develop a theory of photometric stereo for such surfaces. We will address four of the most pressing issues in developing such a theory:

- The Class of Reflectance Maps: The distribution of reflected light depends on the physical mechanisms that cause it. What are the underlying mechanisms? What class of BRDFs and reflectance maps arise from them? Is there an analytically simple way to express the reflectance maps?

Chapter 3 addresses this issue. We identify different mechanisms of reflection from real world surfaces and derive useful reflectance maps from them.

- Inverting the Photometric Stereo Equation: How many light sources do we need to invert the photometric stereo equations 2.8 and 2.7 (see section 2.4)? Of course, we need a globally unique inversion.

Chapters 4 and 5 address these issues. Chapter 4 deals with the minimum number of light sources necessary for inverting the normalized equation while chapter 5 deals with the un-normalized equation.

- Joint Estimation of the Normal and the Reflectance Map: Can the reflectance map of the surface be estimated along with the surface normal? How many light sources do we need to do this?

Chapter 6 addresses the issue of joint estimation of the surface normal and the reflectance map.

- Completeness of reconstruction: If $k$ lights are needed to invert the photometric stereo equation, then every point of the surface must be illuminated by $k$ lights. If only $k$ lights are used and the surface is convex, it is easy to show that some points on the surface lie in the shadow of at least one of the lights. We need $k^{*}$ lights, $\left(k^{*}>k\right)$ in order that at least $k$ of them illuminate every point on the surface. What is the precise relation between $k$ and $k^{*}$ ?

Chapter 7 deals with this issue. 
Experimental evidence is provided for this theory in appropriate chapters. The details of the conversion from surface normals to depth are given in appendix A. A description of the apparatus and the detailed experimental procedure is in appendix B.

Finally, note that competing methods to photometric stereo include structured lighting [9], shape from texture [28], shape from contour [49] and laser ranging techniques. Each of these methods performs well in environments where its underlying assumptions are best met. When compared with these methods, photometric stereo does seem to offer some inherent advantages. For example, it does not need the complex light sources that the structured lighting or laser ranging methods do, nor does it need global information that shape from texture and shape from shading methods [22] do. Since only local information is used, the technique is fast and is often implemented as a table look up. It also provides a unique reconstruction which shape from texture and shading do not. Further, photometric stereo has been successfully used in real-world situations as a visual front end for robotic manipulators [29]. 


\section{Chapter 2}

\section{Background}

\subsection{Introduction}

This chapter provides the background and definitions necessary to develop the theory of photometric stereo presented here. Section 2.2 specifies the notation we will use throughout this work. Section 2.3 contains the definitions that help identify the relation between the surface normal and intensities in an image. Section 2.4 develops the normalized and the unnormalized photometric stereo equations. Section 2.5 briefly introduces the use of extended light sources.

\subsection{Notation}

We will be dealing with quantities like surface normals, light source directions etc. which are vectors in $E^{3}$. These quantities are denoted by bold-face alphabets. Thus, for example, the surface normal is denoted by $\mathrm{n}$. We will also follow the convention that the first component of a vector is the $\mathrm{x}$-component, the second the $\mathrm{y}$-component and the third the $\mathrm{z}$-component. The inner product of any two vectors $\mathbf{x}$ and $\mathbf{y}$ is denoted by $\mathbf{x}^{T} \mathbf{y}$. Since the vectors belong to $E^{3}$ there is a well defined cross product for them and if $\mathbf{z}$ is the result of the cross product of $\mathbf{x}$ and $\mathbf{y}$, then this is denoted by $\mathbf{z}=\mathbf{x} * \mathbf{y}$. If $\mathbf{x}$ is a unit length vector then it can be represented with its zenith and azimuth angles. The zenith and azimuth angles of a unit length vector, e.g. $\mathbf{n}$, will be denoted by $\theta_{n}$ and $\phi_{n}$, the subscript associating the angles with the vector.

In chapter 5 we will have occasion to use some differential geometry in an $E^{2}$ setting. We will follow the standard convention in doing this. A curve in $E^{2}$ is denoted as $\alpha(s)$. Unless specified otherwise, we will assume that the curve is unit speed. The tangent vector to the curve will be denoted by $\alpha^{\prime}(s)$. 


\subsection{The Reflectance Map}

The relation between surface normals and image intensities is understood by exploring its two components: the manner in which the underlying surface reflects light and the manner in which this reflected light is captured and converted to an intensity value by a camera. The first is specified in terms of the Bi-directional Reflectance Distribution Function of the surface and the second is given by Horn's Relationship. The next two sub-sections define and illustrate these relations. For further details the reader is referred to [22] [23].

\section{The Bi-directional Reflectance Distribution Function}

Let us use spherical co-ordinates as shown in figure 2.1. The zenith angle, $\theta$, is positive down from the $z$-axis. The azimuth angle, $\phi$, is positive anticlockwise from the $\mathrm{x}$-axis. The Principle Plane is defined as the plane containing the incident and receiving directions. Monochromatic flux $d \Phi_{i}$ is incident on an area $d A_{i}$ from the direction $\phi_{i}, \theta_{i}$ and a part of it, $d^{2} \Phi_{r}$, is reflected within a solid angle $d \omega_{r}$ in the direction $\phi_{r}, \theta_{r}$. The Irradiance $E_{i}$ on the surface is defined as

$$
E_{i}=\frac{d \Phi_{i}}{d A_{i} \cos \theta_{i}},
$$

and the Radiance from the surface is defined as

$$
L_{r}=\frac{d^{2} \Phi_{r}}{d A_{i} \cos \theta_{r} d \omega_{r}} .
$$

The BRDF (Bi-directional Reflectance Distribution Function) ${ }^{1}$ of the surface is

$$
f_{r}\left(\theta_{i}, \phi_{i} ; \theta_{r}, \phi_{r}\right)=\frac{L_{r}}{E_{i}}
$$

If the reflecting surface is isotropic, we can align the $\mathrm{x}$-axis of our reference frame with the incident azimuth direction $\phi_{i}$ and drop the dependence of $f_{r}()$ on $\phi_{i}$. Then, $f_{r}()$ has the form $f_{r}\left(\theta_{i} ; \theta_{r}, \phi_{r}\right)$.

All of the light incident on a perfect plane mirror from any direction $\theta_{i}, \phi_{i}$, is reflected in the direction $\theta_{r}=-\theta_{i}, \phi_{r}=\phi_{i}$. In this case, the BRDF consists of a

\footnotetext{
${ }^{1}$ In general, A BSSRDF (Bi-directional Scattering Surface Reflectance Distribution Function) has to be defined to describe reflection from translucent material [40]. For opaque surfaces, the definition of BSSRDF reduces to that of the BRDF.
} 


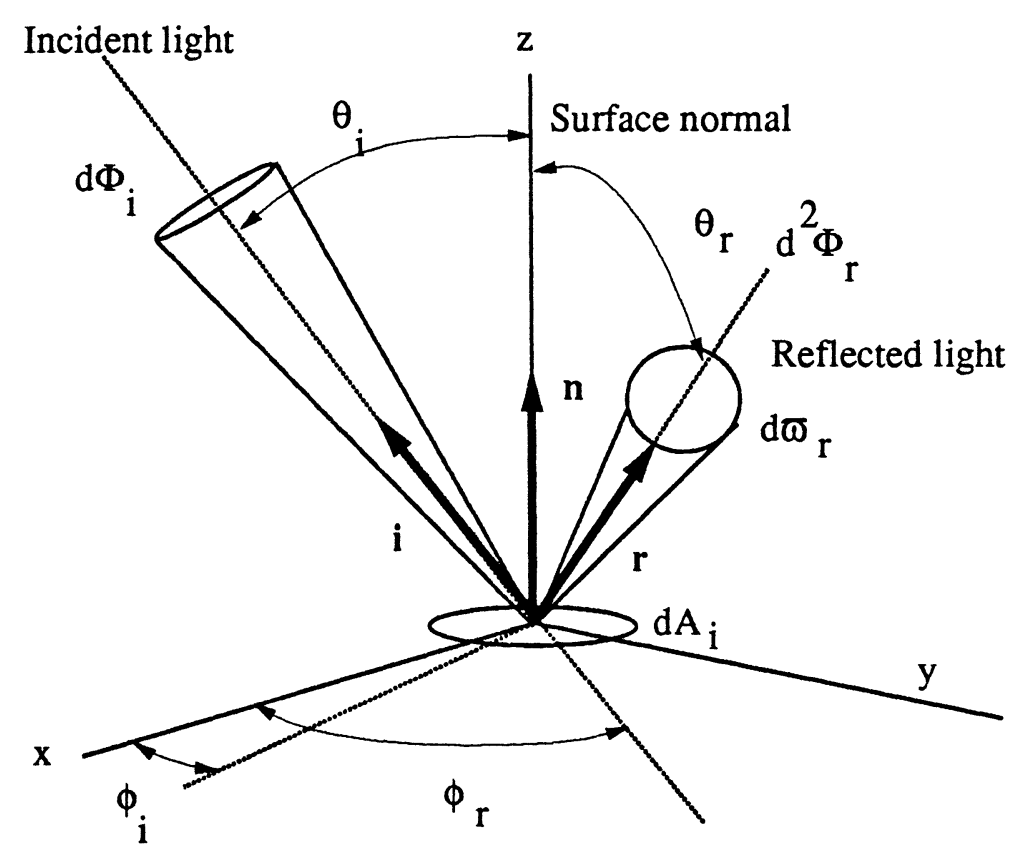

Figure 2.1 The bi-directional reflectance distribution function

delta function which is zero everywhere except along $\theta_{r}=-\theta_{i}, \phi_{r}=\phi_{i}$. In general, the BRDF is a sum of delta functions and functions of bounded variation. The delta functions are attributed to specular reflection and the rest to diffuse reflection. For a large class of surfaces, the BRDF has only one specular term and its delta function is non-zero along $\theta_{r}=-\theta_{i}, \phi_{r}=\phi_{i}$, which is its specular direction.

The term "specular reflection" has not been used consistently in the literature. Ikeuchi [27] uses the term in the sense that we have used it above. However, Horn [21] and Healy [19] use it in a different sense. They use the term to denote the diffuse reflection that is scattered around the specular direction. It is obvious from the form of the specular term they use that it is a function of bounded variation and is non-zero in a whole region of space. This confusion in the nomenclature can be avoided by adopting the following convention: we reserve the term "specular reflection" for that part of the BRDF which is denoted by delta functions. The diffuse lobe around the specular direction (see chapter 3 ) is termed the "forescatter lobe".

Light is a form of energy and reflection is governed by the laws of thermodynamics. The second law of thermodynamics requires that the following relation, called 
Helmholtz's reciprocity relation, hold for all BRDFs [22]:

$$
f_{r}\left(\theta_{i}, \phi_{i} ; \theta_{r}, \phi_{r}\right)=f_{r}\left(\theta_{r}, \phi_{r} ; \theta_{i}, \phi_{i}\right) \text {. }
$$

The BRDF is often multiplied by an albedo term $\rho$ and written as $\rho . f_{r}$. Surfaces differing in their albedos have the same profile of reflected light but differ in the net amount of reflected light.

While comparing different BRDFs, it is necessary to account for albedo variations. To do this, the BRDF is usually presented as a plot of the ratio $\frac{f_{r}\left(\theta_{i} ; \theta_{r}, \pi\right)}{f_{r}\left(\theta_{i} ; \theta_{i}, \pi\right)}$ as a function of $\theta_{r}$ for different $\theta_{i}$. Thus, the BRDF curve is forced to have a value of 1 in the specular direction. See figure 3.4. One of the older conventions for presenting BRDF is to plot $f_{r}() \cos \theta_{r}$ as a function of $\theta_{r}$ in a polar plot. Some of the data that we present is plotted in this fashion.

Sometimes, it is convenient to express the BRDF in parameters other than $\theta_{i}, \theta_{r}$ and $\phi_{r}$. Horn uses the gradient space [22]. We will use the unit norm vectors $\mathbf{n}, \mathbf{i}, \mathbf{r}$ shown in figure 2.1. Here, $\mathbf{n}$ is the surface normal vector, $\mathbf{i}$ is the illumination direction, and $\mathbf{r}$ is the receiving direction. Note that $\mathbf{n}^{T} \mathbf{n}=\mathbf{i}^{T} \mathbf{i}=\mathbf{r}^{T} \mathbf{r}=1$. Using the unit norm vectors, the BRDF is written as $f_{r}(\mathbf{n}, \mathbf{i}, \mathbf{r})$. The angle between $\mathbf{i}$ and $\mathbf{r}$ is called the phase angle.

Most surfaces reflect some of the incident light diffusely. It is commonly assumed that diffuse reflection from any surface obeys Lambert's law.

Lambert's Law: Lambert's law states that diffuse reflection has a constant BRDF, i.e.

$$
f_{\text {diff }}(\mathbf{i}, \mathbf{n}, \mathbf{r})=\frac{1}{\pi}
$$

As yet, there is no mechanism that has been isolated which completely explains this law. The usual "explanation" the law is [39]: Incident light penetrates in to the bulk of the material. Inside, it undergoes repeated reflection causing the emergent radiation to be uniformly distributed. The BRDF for uniformly emergent radiation can be shown to obey Lambert's law.

Note that this is not really an explanation - it is simply a restatement of the law. Any explanation of Lambert's law has to explain why the distribution of emergent radiation is independent of the angle of incidence of the incident radiation. In fact, it is possible to show (see section 3.6) that multiple reflection can cause non-Lambertian diffuse reflection. 
Strictly speaking, within the bulk of an object, light does not reflect but is scattered. There is a strong connection between scattering and reflection, and as we shall see in chapter 3 , reflection is organized scattering. In the literature, the terms scattering and reflection are used interchangably and we will continue to do so in this thesis.

\section{Horn's Relation.}

Figure 2.2 shows how image intensities are formed. Let us assume that the object is far enough from the image plane to use parallel projections. Also assume that the light source is far enough to be approximated as a point source at infinity. For this geometry, as $\mathbf{r}$ lies along the $\mathrm{z}$-axis, the principal plane always contains the $\mathrm{z}$-axis. Consider a point on the surface of the object that has a surface normal $\mathbf{n}$ as shown. The image of this point is formed at $\mathrm{x}, \mathrm{y}$ in the image plane. Let $d A$ be a small surface patch around this point. Horn [22] proved that for this geometry, if the surface is convex, the recorded intensity in the image, $I(x, y)$, is proportional to the radiance of $d A$ along $\mathbf{r}$. Thus, if $L(x, y)$ is the radiance of the point $x, y$ along $\mathbf{r}$, then Horn's relation states that

$$
I(x, y)=\alpha L(x, y)
$$

where, $\alpha$ is the constant of proportionality in Horn's relation.

If $E$ is the irradiance of the light source in the direction $\mathbf{i}$, then the radiance of $d A$ along $\mathbf{r}$ is

$$
L(x, y)=E \rho(x, y) f_{r}(\mathbf{n}(x, y), \mathbf{i}, \mathbf{r}) \mathbf{n}^{T}(x, y) \mathbf{i},
$$

where, $\rho(x, y)$ is the albedo on the surface at the point $\mathrm{x}, \mathrm{y}$.

From Horn's relation and the above equation we get the intensity at any point $x, y$ in the image as

$$
I(x, y)=a R(\mathbf{i}, \mathbf{n}(x, y), \mathbf{r}) .
$$

where $a=E \rho \alpha$, and $R()$ is called the Reflectance Map.

$$
R(\mathbf{i}, \mathbf{n}, \mathbf{r})=\mathbf{i}^{T} \mathbf{n} f_{r}(\mathbf{i}, \mathbf{n}, \mathbf{r}) .
$$

For a unity albedo Lambertian surface,

$$
R(\mathbf{i}, \mathbf{n}, \mathbf{r})=\frac{1}{\pi} \mathbf{i}^{T} \mathbf{n} \quad \ldots \text { if } \mathbf{i}^{T} \mathbf{n}>0
$$




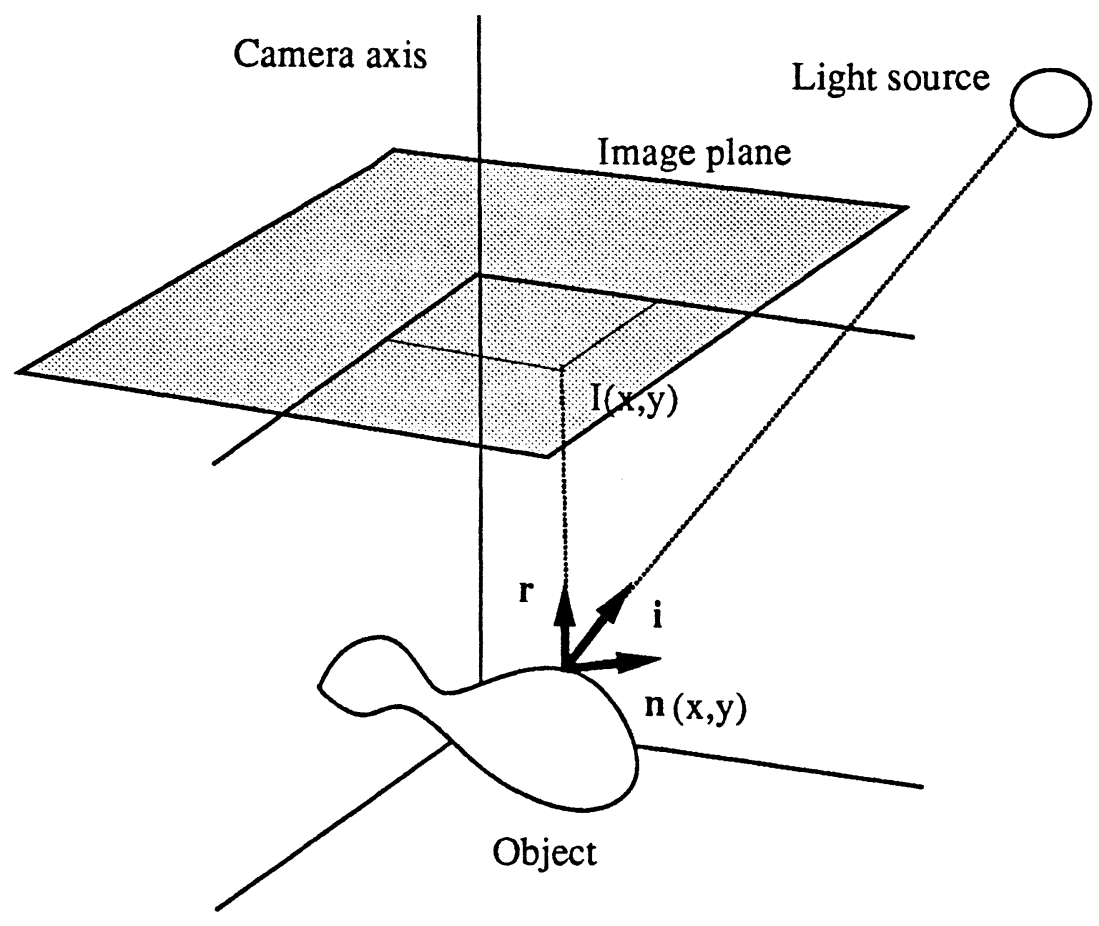

Figure 2.2 Illustration for Horn's relation

$$
=0 . \quad \ldots \text { otherwise. }
$$

For a unity albedo specular surface,

$$
R(\mathbf{i}, \mathbf{n}, \mathbf{r})=\zeta \delta\left(1-\mathbf{n}_{\mathbf{s}}{ }^{T} \mathbf{n}\right) .
$$

where, $\mathbf{n}_{\mathrm{s}}$ is called the specular direction and is the orientation of the surface at which specular reflection from the surface reflects into the camera

$$
\mathbf{n}_{\mathbf{s}}=\frac{\mathbf{i}+\mathbf{r}}{\|\mathbf{i}+\mathbf{r}\|}
$$

and, $\zeta$ is a constant with respect to $\mathbf{n} . \delta()$ is the Dirac delta function.

Note that if $\mathbf{i}^{T} \mathbf{n} \leq 0$ then $\mathbf{n}$ lies in its own shadow with respect to $\mathbf{i}$. This condition is termed as self-shadow. 


\subsection{The Photometric Stereo Equations}

The main idea behind photometric stereo is to invert equation (2.4) from a priori knowledge of the reflectance map and measurement of the intensity in the image. Note that $\mathbf{n}$ is constrained by $\mathbf{n}^{T} \mathbf{n}=1$. Thus, there are two unknowns in $\mathbf{n}$ and $\mathbf{a}$ single equation of the type (2.4) cannot be used to obtain a unique $\mathbf{n}$. More than one intensity value is required and this is achieved by using more than one light source. See figure 1.2.

If we keep the relative position between the camera and the object the same and use $k$ lights from the directions $\mathrm{i}_{l}, l=1,2, . ., k$ to illuminate the surface, one light source at a time, then the $k$ different image intensities for any pixel are given by

$$
\left(\begin{array}{c}
I_{1}(x, y) \\
\ldots \\
I_{k}(x, y)
\end{array}\right)=\left(\begin{array}{c}
a_{1} R\left(\mathbf{i}_{1}, \mathbf{n}, \mathbf{r}\right) \\
\cdots \\
a_{k} R\left(\mathbf{i}_{k}, \mathbf{n}, \mathbf{r}\right)
\end{array}\right) .
$$

If we assume that all the light sources have the same strength then, $a_{1}=a_{2}=\cdots=$ $a_{k}=a$ and the above equation can be written as:

$$
\left(\begin{array}{c}
I_{1}(x, y) \\
\cdots \\
I_{k}(x, y)
\end{array}\right)=a\left(\begin{array}{c}
R\left(\mathbf{i}_{1}, \mathbf{n}, \mathbf{r}\right) \\
\ldots \\
R\left(\mathbf{i}_{k}, \mathbf{n}, \mathbf{r}\right)
\end{array}\right) .
$$

We call this equation the Un-normalized Photometric Stereo Equation.

Following Horn [22], if the maximum value of intensity in the $j^{\text {th }}$ image is $I_{m a x}, j$, then defining $I_{j}^{r e l}(x, y)=I_{j}(x, y) / I_{m a x, j}$, we get:

$$
\left(\begin{array}{c}
I_{1}^{r e l}(x, y) \\
\ldots \\
I_{k}^{r e l}(x, y)
\end{array}\right)=\left(\begin{array}{c}
R\left(\mathbf{i}_{1}, \mathbf{n}, \mathbf{r}\right) \\
\ldots \\
R\left(\mathbf{i}_{k}, \mathbf{n}, \mathbf{r}\right)
\end{array}\right) .
$$

We will call this the Normalized Photometric Stereo Equation.

For purely Lambertian surfaces, using three light sources from the directions $\mathbf{i}_{1}$, $\mathbf{i}_{2}$ and $\mathbf{i}_{3}$, equation (2.7) reduces to $\left(I_{1} I_{2} I_{3}\right)^{T}=a\left(\mathbf{i}_{1}^{T} \mathbf{i}_{2}^{T} \mathbf{i}_{3}^{T}\right)^{T} \mathbf{n}$. This equation is usually rewritten as $\left(I_{1} I_{2} I_{3}\right)^{T}=\left(\mathbf{i}_{1}^{T} \mathbf{i}_{2}^{T} \mathbf{i}_{3}^{T}\right)^{T} \mathbf{N}$, where, $\mathbf{N}=a \mathbf{n}$. This is a linear equation and can be solved for $N$. From $\mathbf{N}$, we get $a=\|\mathrm{N}\|$ and $\mathbf{n}=\mathrm{N} / a$. Most of the theoretical work in photometric stereo [60] [42] [15] has been done using this Lambertian model.

Before we proceed, we will define some more terminology. Note that we are interested in reconstructing only those surface normals that are visible i.e. we are 
interested in surface normals that lie in the set $S$,

$$
S=\left\{\mathbf{n}: \mathbf{n}^{T} \mathbf{z}>0, \mathbf{z}=\left[\begin{array}{lll}
0 & 0 & 1
\end{array}\right]^{T}\right\}
$$

Given any light sources $\mathrm{i}_{1}, \mathbf{i}_{2}, \cdots, \mathbf{i}_{k}$, in general not all elements of $S$ are illuminated by every light source. Let $O$ be the set of normals that are illuminated by the $k$ light sources,

$$
O=\left\{\mathbf{n}: \mathbf{n}^{T} \mathbf{i}_{l}>0, l=1, \cdots, k\right\}
$$

We call $O$ the set of reconstructible normals.

The photometric stereo equation has to be solved for each point on the surface, and for each point we would like a unique solution. By, a Globally Unique Solution of the photometric stereo stereo equation we mean that for any $\mathbf{n}_{1} \in O$, we cannot find another $\mathbf{n}_{2} \in S, \mathbf{n}_{1} \neq \mathbf{n}_{2}$ which yield the same vector for the left hand side of equation 2.7 and 2.8. We use the term globally unique solution to distinguish it from the results of the type obtained by applying the inverse function theorem which yield a Locally Unique Solution, i.e. which prove that given a solution $\mathbf{n}$ to the photometric stereo equation, there is no other solution to the same equation within some neighborhood of $\mathbf{n}$ in $O$. Usually, the application of the inverse function theorem yields a neighborhood which is a proper subset of $S$. In terms of practically reconstructing a real-world surface such results are not strong enough. We have to demand a globally unique reconstruction. Typically, we need more light sources to obtain a globally unique solution as compared to a locally unique solution. As Woodham [60] has shown, for the Lambertian case, a locally unique solution can be obtained by using two light sources, but three light sources are needed for a globally unique solution.

Once we obtain conditions under which a globally unique solution can be obtained for elements of $O$, we consider modifying the conditions such that the photometric stereo equation can be solved for all elements of $S$, i.e. we will use $k^{*}, k^{*}>k$ light sources in such a fashion that any $\mathbf{n} \in S$, is contained in the set of reconstructible surface normals of some $k$ of these light sources. If we can find such conditions, then the surface can be reconstructed completely, and we call such a reconstruction a Complete Reconstruction.

Finally, it is worth mentioning that the benchmark test of any photometric stereo method is the reconstruction of a spherical surface. Such a surface has normals in 
every direction and reconstructing such a surface demonstrates the fidelity of the method over the entire range of surface normals.

Appendix A provides the details of converting surface normals into depth values.

\subsection{Extended Light Sources}

This work does not deal with extended light sources, but some of their properties are of interest to us and in this section, we will very briefly consider them. Extended light sources are light sources that are spread over an entire region of space, typically the whole hemisphere above the object. Light from the sky is an excellent example of this. With respect to the object, extended light sources are specified in terms of the radiance emmited by them per unit solid angle. The radiance per unit solid angle is denoted by $E\left(\theta_{i}, \phi_{i}\right)$.

Figure 2.3 shows a light source extended over a hemisphere on top of the surface to be reconstructed. We assume as usual that the direction $\mathbf{r}$ is vertical. Following Horn [22] let us consider a patch of the extended light source in the direction $\theta_{i}, \phi_{i}$. It can be shown that the irradiance from this patch to a surface normal oriented along $\theta_{n}, \phi_{n}$ is

$$
E\left(\theta_{i}, \phi_{i}\right) \sin \theta_{i}\left(\cos \theta_{i} \cos \theta_{n}+\sin \theta_{i} \sin \theta_{n} \cos \left(\phi_{i}-\phi_{n}\right)\right) \delta \theta_{i} \delta \phi_{i} .
$$

Hence, the contribution of this patch to the radiance along $\mathbf{r}$ is

$$
f\left(\theta_{i}^{\prime}, \phi_{i}^{\prime} ; \theta_{n}, 0\right) E\left(\theta_{i}, \phi_{i}\right) \sin \theta_{i}\left(\cos \theta_{i} \cos \theta_{n}+\sin \theta_{i} \sin \theta_{n} \cos \left(\phi_{i}-\phi_{n}\right)\right) \delta \theta_{i} \delta \phi_{i}
$$

where, $f()$ is the BRDF of the surface which is assumed to be isotropic and $\theta_{i}^{\prime}$ and $\phi_{i}^{\prime}$ are the zenith and azimuth angles of the extended light patch along the direction $\theta_{i}, \phi_{i}$ with respect to the surface normal.

$$
\begin{aligned}
\cos \theta_{i}^{\prime} & =\cos \theta_{i} \cos \theta_{n}+\sin \theta_{i} \sin \theta_{n} \cos \left(\phi_{i}-\phi_{n}\right), \\
\sin \phi_{i}^{\prime} & =\sin \theta_{i} \frac{\sin \left(\phi_{i}-\phi_{n}\right)}{\sin \theta_{i}^{\prime}} .
\end{aligned}
$$

The net radiance along $\mathbf{r}$ is given as

$$
\begin{array}{rl}
L\left(\theta_{n}, \phi_{n}\right)=\int_{-\pi}^{\pi} \int_{0}^{\pi / 2} & f\left(\theta_{i}^{\prime}, \phi_{i}^{\prime} ; \theta_{n}, 0\right) E\left(\theta_{i}, \phi_{i}\right) \\
& \sin \theta_{i}\left(\cos \theta_{i} \cos \theta_{n}+\sin \theta_{i} \sin \theta_{n} \cos \left(\phi_{i}-\phi_{n}\right)\right) \delta \theta_{i} \delta \phi_{i}
\end{array}
$$

Using Horn's relationship, we have the intensity $I()$ as a function of $\mathbf{n}$ as 


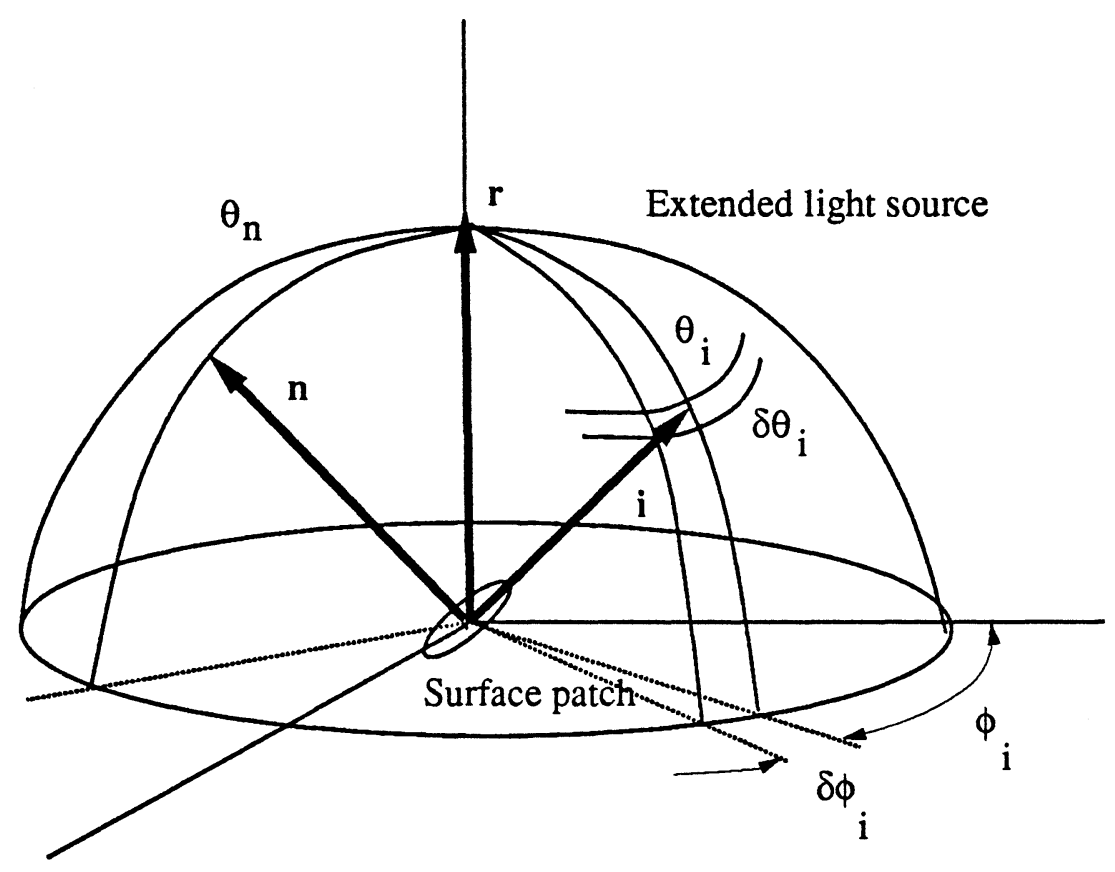

Figure 2.3 Extended light source

$$
\begin{aligned}
I\left(\theta_{n}, \phi_{n}\right)=\alpha \int_{-\pi}^{\pi} \int_{0}^{\pi / 2} \quad & f\left(\theta_{i}^{\prime}, \phi_{i}^{\prime} ; \theta_{n}, 0\right) \\
& E\left(\theta_{i}, \phi_{i}\right) \sin \theta_{i}\left(\cos \theta_{i} \cos \theta_{n}+\sin \theta_{i} \sin \theta_{n} \cos \left(\phi_{i}-\phi_{n}\right)\right) \delta(2 \delta \phi)
\end{aligned}
$$

The integral on the right hand side yields the reflectance map [22].

At this point, we will summarize the literature on extended light sources [22] [27] [39] with the following comments

1. Extended light sources were first used on purely specular surfaces [27]. When rotationally symmetric extended light sources are used on a specular surface, the resulting reflectance map looks like a diffuse reflecting map [39]. In fact, the resulting map is very similar to the forescatter reflectance map that we will propose for diffuse reflection from rough surfaces in chapter 3.

2. Note that for an extended light source, $E()$, the radiance per unit solid angle is a non-negative finite quantity. Thus the integration in equation (2.9) "smooths out" the BRDF $f()$ to yield the net reflectance map. Extended light sources 
have been used explicitly for the purpose of low-pass filtering the reflectance map, i.e., to reduce the frequency content of $I()$ when viewed as a function of $\theta_{n}$ or $\phi_{n}[39]$.

3. Extended light sources can be moved just as point light sources can be and the fact that they are extended makes the variation of $I()$ with respect to a change in their position a smoother (low pass) function [39] too. An example will make this clear. Consider the difference in the illumination produced by the changing position of the sun in a clear sky and in an overcast sky. The changing position of the sun in an overcast sky effectively changes the pattern of the diffuse light emmited by the sky, and this change has a far gentler effect in changing the shading of objects on the earth as compared to the change in shading due to sun in a clear sky where bulk of the radiation incident on earth is not diffuse. We will refer to this smoothing property of diffuse surfaces in chapter 6 . 


\section{Chapter 3}

\section{Reflection from Solids}

\subsection{Introduction}

In this chapter, we will investigate the nature of reflection from real-world solid surfaces. The BRDF for most surfaces can be written as a linear combination of specular and diffuse terms. Previous work [60] [42] [15] [39] had assumed that diffuse reflection occurs in a single lobe. In the present chapter we propose a theory, based on experimental evidence, which assumes that diffuse reflection occurs in three lobes. Each lobe gives rise to a term in the BRDF. We term the lobes: the Forescatter Lobe, the Normal Lobe and the Backscatter Lobe. The main aim of this chapter is to investigate the nature of the lobes: the mechanisms which cause them, the nature of the terms that represent them in the BRDF, etc.

Before we proceed, some cautionary remarks are in order. Reflection from realworld surfaces is not a perfectly understood phenomenon and still the subject of much research [1] [2]. Theories which explain the diffuse lobes are approximate. Of the three lobes, the forescatter lobe has been the most widely studied, the normal lobe a little less and the backscatter lobe scantly. In some cases, very little is known about modeling the reflection mechanism, and we are forced to seek analogies and postulate functional forms for BRDFs from empirical observations of published data and theories. However, there are reasons to believe that the class of reflectance maps proposed in this chapter model real data very well. First, the theories presented in this chapter explain the experimental data from a wide range of publications - data from metallic and dielectric rough surface reflection, atmospheric optics, backscattering from paints, etc. Second, the theories can explain the origin of Lambert's law. Third, the reflectance maps that these theories predict, model actual image data very well. Finally, the reflectance maps are intuitively plausible and have a nice geometric interpretation.

This chapter is structured as follows. Section 3.2 contains a brief preview of our model. It identifies the lobe structure of diffuse reflection. A physical explanation 
of the origin of the lobes follows in subsequent sections. As we shall see, these lobes come about because of surface and bulk inhomogeneities of any solid. A perfect homogeneous solid reflects only in a specular fashion. Section 3.3 explains the origin of specular reflection from a perfect solid. Sections 3.4 onward deal with inhomogeneous solids and how the inhomogeneity causes diffuse lobes. Section 3.5 explains the forescatter lobe, section 3.6 the normal lobe and section 3.7 the backscatter lobe. Section 3.8 combines the results of the previous sections and derives the reflectance map for a surface that reflects in all the modes. Certain approximations can greatly simplify the form of the reflectance map and section 3.8 uses these approximations. As it turns out, the form of reflectance map that we present in section 3.8 has interesting mathematical properties that lead to a generalization of the map. This generalization and the model that arises out of it is considered in section 3.9. Finally, in section 3.10, we present brief experimental evidence that the reflectance map of section 3.8 models actual image data better than the specular-Lambertian model.

\subsection{Scattering Lobes and Rough Surfaces.}

Figure 3.1 shows an incident ray being reflected off a surface. As shown in the figure, part of the ray is specularly reflected and part of it is diffusely reflected. The forescatter lobe of the diffuse reflection is the part which is spread around the specular direction. The normal lobe is spread around the surface normal and the backscatter lobe around the incidence direction. Mathematically, our proposed classification splits the BRDF as:

$$
\begin{aligned}
f_{r}\left(\theta_{i} ; \theta_{r}, \phi_{r}\right)= & f_{s p e c}\left(\theta_{i} ; \theta_{r}, \phi_{r}\right) \\
& +f_{f s c}\left(\theta_{i} ; \theta_{r}, \phi_{r}\right)+f_{\text {norm }}\left(\theta_{i} ; \theta_{r}, \phi_{r}\right)+f_{b s c}\left(\theta_{i} ; \theta_{r}, \phi_{r}\right) .
\end{aligned}
$$

where, $f_{\text {spec }}()$ is the specular term, $f_{f s c}()$ due to the forescatter lobe, $f_{\text {norm }}()$ due to the normal lobe and $f_{b s c}()$ due to the backscatter lobe.

Let us call the specular, normal and the incident directions as principal directions. A principal direction is one around which a diffuse lobe is spread. In general, the reflected light can spread around many principal directions. Beckmann [6] contains evidence of this.

As we will see in section 2.5, the forescatter lobe is closely connected with specular reflection. For ease of discussion, it is better to include specular reflection as a part 


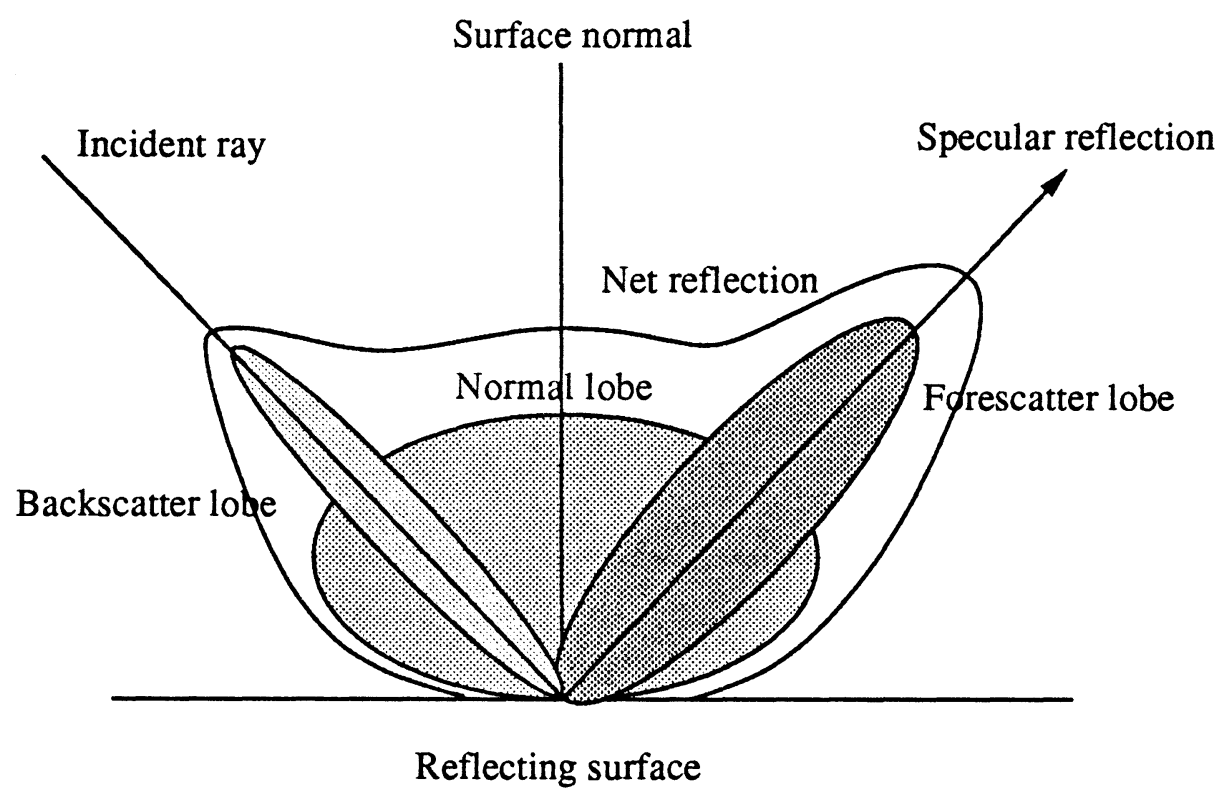

Figure 3.1 The structure of reflected light

of the forescatter lobe. We shall do this in section 2.5 and refer to it as the specular part of the forescatter lobe.

Figure 3.2 shows the BRDF plotted radially for Zinc Oxide paint [10]. The three lobes are easily identifiable. This shape of the BRDF curve occurs repeatedly in all the available data [54] [53] [52] [37] [36] [26].

Horn [21] and Silver [47] have suggested that realistic reflectance maps for diffuse reflection can be constructed as the sum of a forescatter and a normal term.

$$
R(\mathbf{i}, \mathbf{n}, \mathbf{r})=d R_{n o r m}(\mathbf{i}, \mathbf{n}, \mathbf{r})+(1-d) R_{f s c}(\mathbf{i}, \mathbf{n}, \mathbf{r}),
$$

where, $d$ is a real number between 0 and 1 . Silver's suggestion for the components is:

$$
R_{\text {norm }}(\mathbf{i}, \mathbf{n}, \mathbf{r})=\mathbf{i}^{T} \mathbf{n}
$$

and,

$$
R_{\text {spec }}(\mathbf{i}, \mathbf{n}, \mathbf{r})=S(\mathbf{i}, \mathbf{n}, \mathbf{r})
$$

where, $S()$ is an unspecified standard specular reflectance map. 


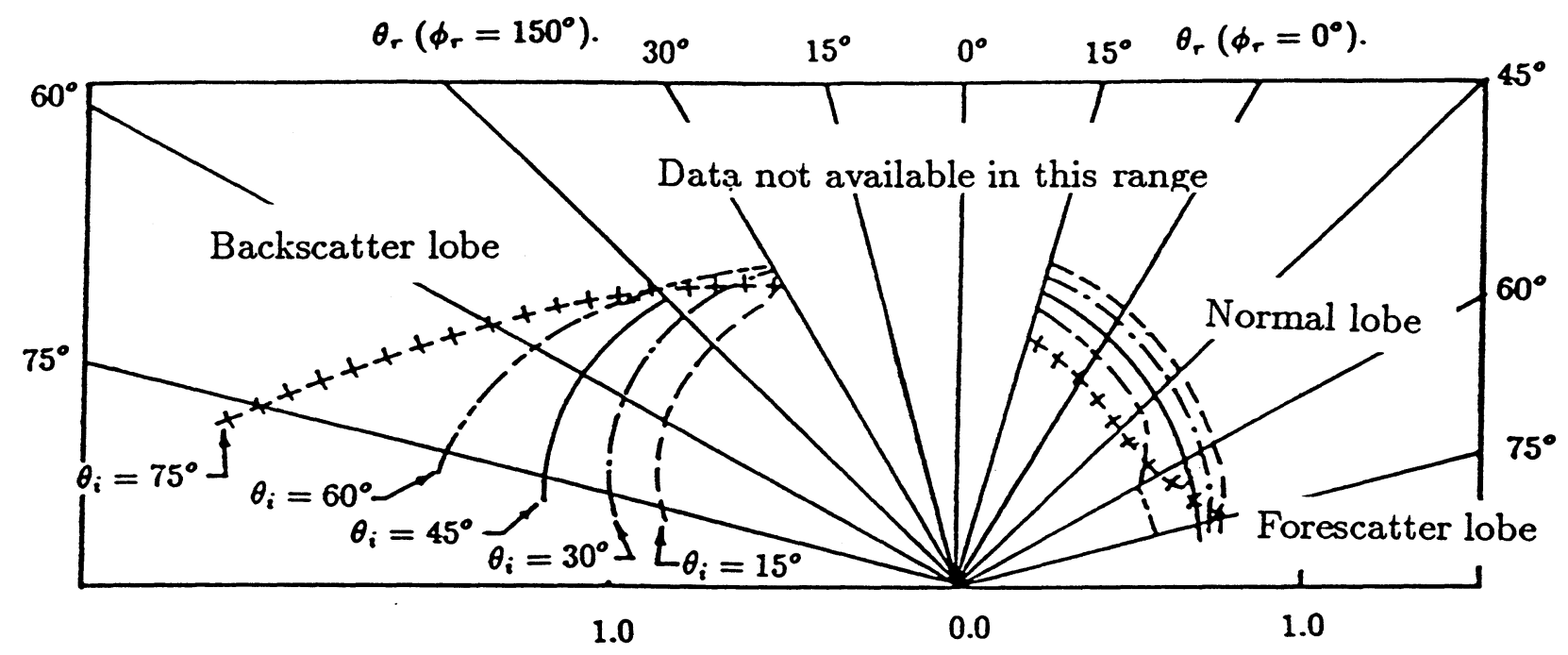

Arbitrarily scaled $f_{r}()$.

Figure 3.2 BRDF showing three diffuse lobes

Horn's suggestion is the same as above, except that he uses a specific function $S():$

$$
S(\mathbf{i}, \mathbf{n}, \mathbf{r})=\left(\mathbf{n}_{\mathbf{s}}{ }^{T} \mathbf{n}\right)^{\gamma} .
$$

where, $\gamma$ is a positive real number and $\mathrm{n}_{\mathbf{s}}$ is given by equation (2.10).

Using this expression for the reflectance map, the BRDF is obtained as

$$
f_{r}(\mathbf{i}, \mathbf{n}, \mathbf{r})=d+(1-d) \frac{\left(\mathbf{n}_{\mathbf{s}}{ }^{T} \mathbf{n}\right)^{\gamma}}{\left(\mathbf{i}^{T} \mathbf{n}\right)} .
$$

Note that this BRDF does not obey Helmholtz's reciprocity. Thus Horn's reflectance map cannot arise out of any physical model of reflection. One of the achievements of the theories proposed in this chapter is to provide some justification for these reflectance maps. As we will see in section 3.S, these reflectance maps are valid because they are mathematical approximations to BRDFs that do obey Helmholtz's reciprocity. We lose strict reciprocity in the process of approximation. 


\subsection{The Basic Theories of Scattering and Specular Reflection}

Perfect solids are composed of regular arrays of the constituent molecules or atoms. An understanding of how such solids reflect is achieved by analyzing how individual molecules or atoms scatter and how that behavior is modified by their being together in a dense regular array.

Consider scattering from isolated particles. This was first studied by Rayleigh and is called Rayleigh scattering. We refer the reader to [56] [34] [4] for a complete treatment of single particle scattering. The distribution of light scattered from a point scatterer is described by a Phase Function $p(\cos \Theta)$, where $\Theta$ is the phase angle. Two common models of scattering from individual particles are that of isotropic scattering and Rayleigh scattering. The phase function for the isotropic scatterer is

$$
p(\cos \Theta)=\omega
$$

where $\omega$ is a constant, $0 \leq \omega \leq 1.0$. The phase function for a Rayleigh scatterer is

$$
p(\cos \Theta)=\frac{3}{4}\left(1+\cos ^{2} \Theta\right) .
$$

When identical scattering particles are put in a low density cluster, the net amount of scattering increases but the scattering profile of the cluster remains the same as that of the individual scatterer. As the cluster density increases, Multiple Scattering occurs. Each scatterer is illuminated by the neighboring scattered radiation as well as the incident light. The resulting scattering profile differs substantially from that of the single particle. As the density of the cluster increases further and the distance between any two scatterers becomes comparable to the wavelength of incident light, the medium becomes Optically Dense. As the incoming wave propagates through the dense medium, the scatterers in the medium get coupled by their oscillating fields. This creates a secondary wave which interferes with the incident wave. This theory was proposed by Ewald and Oseen and the reader is referred to [50] for a good qualitative treatment. The Ewald-Oseen theory predicts that for a homogeneous optically dense medium:

(1) In the medium, the incident and the secondary waves interfere to yield a wave that propagates in a different direction than the incident wave. This new direction is the same as that given by Snell's Law of refraction.

(2) Outside the medium, the interference causes specular reflection. 
Thus, perfect solids can only reflect specularly. Classically, the nature of this reflection is obtained by solving Maxwell's equations with the appropriate boundary conditions. Such a treatment can be found in any good reference on optics, e.g., [32] [50] [46].

The Fresnel Reflectivity $F\left(\theta_{i} ; \eta\right)$ of a plane surface is defined as the ratio of the reflected energy to the incident energy, where, $\eta$ is the Complex Index of Refraction of the medium and $\theta_{i}$ is the zenith angle of the incident direction. Analytic forms for the Fresnel Reflectivity $F\left(\theta_{i}, \eta\right)$ are available in [46]. Fresnel reflectivity is approximately 1 for most metals. For dielectrics, it starts at a low value and increases to 1 with increasing $\theta_{i}$. The Fresnel reflectivities of metals and dielectrics are approximately constant in the range $0 \leq \theta_{i} \leq 40^{\circ}$.

\subsection{Inhomogeneous Solids}

Real world solids are not perfect. At the scale of the wavelength of visible light their surfaces are rough and their bulk is inhomogeneous. These imperfections cause light to be reflected in non-specular directions. The geometric distribution of diffuse reflection depends on the nature and distribution of surface roughness and bulk inhomogeneities. If there is no pattern to the roughness of the surface and to the bulk inhomogeneities, then there is no organized pattern to the reflected light - it is diffusely reflected in all regions of space. The aim of this section is to investigate the nature of these inhomogeneities.

\section{Rough Surfaces}

A rough surface can be mathematically described as a two dimensional stochastic process $z(x, y)$, where $z$ is the height of the surface at any point $x, y$. The process $z()$ is usually assumed to have the following properties [6]:

1. It is stationary and has zero mean.

2. It is ergodic.

3. $R(\chi, \gamma)$, the autocorrelation function of $z()$, is circularly symmetric i.e. $R(\chi, \gamma)$ is a function of $r, r=\left(\chi^{2}+\gamma^{2}\right)^{\frac{1}{2}}$. This requires the surface to be isotropic.

4. The autocorrelation function is a strictly decreasing function of $r$. Such surfaces do not have height variations that persist in being similar over long distances. 
Most rough surface reflection theories further assume that the surface is Gaussian and has a Gaussian autocorrelation [6] [54]. At any point $x, y$ the height $z$ is distributed according to

$$
p_{z}(z)=\frac{1}{\sigma \sqrt{2 \pi}} e^{-\frac{z^{2}}{2 \sigma^{2}}}
$$

The autocorrelation function $R(r)$ is assumed to have the form :

$$
R(r)=\sigma^{2} e^{\frac{-r^{2}}{\rho^{2}}}
$$

where $\rho$ is called the correlation length of the surface. ${ }^{1}$ For such surfaces, Beckmann [6] has shown that the distribution of the angle of the slope of the surface is also approximately Gaussian. If $\psi$ is the local angle of inclination of the surface with the normal to the mean surface, then,

$$
p_{\psi}(\psi)=\frac{\rho}{2 \sigma \sqrt{\pi} \cos ^{2} \psi} e^{-\frac{\rho \tan ^{2} \psi^{2}}{4 \sigma^{2}}} .
$$

If $\rho>2 \sigma$ then the distribution can be approximated as:

$$
p_{\psi}(\psi)=\frac{1}{\beta_{o} \sqrt{\pi}} e^{-\frac{\psi^{2}}{\beta_{o}^{2}}}
$$

where, $\tan \beta_{o}=\frac{2 \sigma}{\rho}$.

Before we pass on, let us briefly look at some of the experimental evidence that supports this rough surface model. Funai and Rolling [17] studied various methods such as rolling and shot blasting which are used to create surfaces in a machine shop. They report that all techniques of surface formation do not give isotropic surfaces. Rolled sheet metal, for example, has an r.m.s.roughness value between 0.05 and 0.125 micrometers in the direction normal to the roll marks and less than 0.025 micrometers in the direction parallel to them. The surfaces that best seem to fit the isotropic random process assumption are shot-blasted and closely machined surfaces.

Varnier, Rasigni, et al. [57] investigated height and slope distributions of metallic deposits. In figure 3.3 we show one of their experimental results for height and slope distributions and the corresponding Gaussian fits. Note that the Gaussian model is a fairly good assumption. Also note that the deviation of this surface from being

\footnotetext{
${ }^{1} \mathrm{An}$ allied term is optical roughness Let monochromatic light of wavelength $\lambda$ be incident on a rough surface whose height variance is $\sigma$. The optical roughness of the surface is defined as the ratio $\frac{\sigma}{\lambda}$.
} 


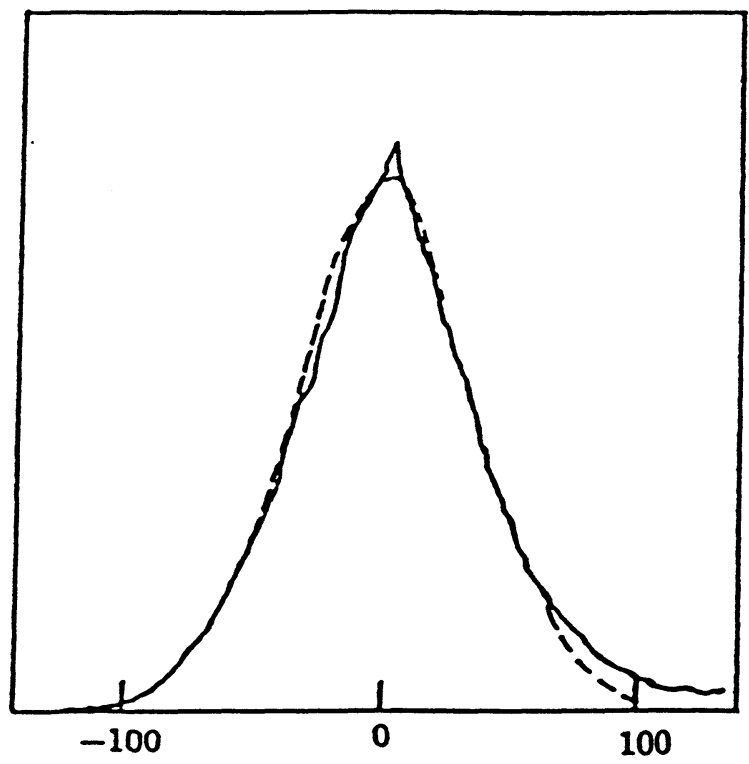

Distribution of height from mean surface $\left(A^{\circ}\right)$.

-- - Gaussian Fit.

Experimental data.

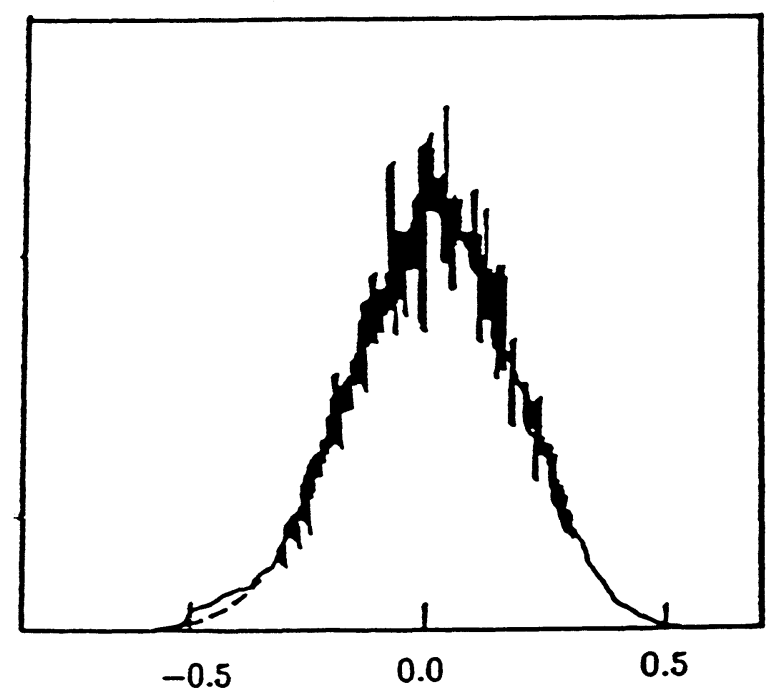

Tangent of the inclination angle.

Figure 3.3 Height and slope distributions. Adapted from [57]. 
a perfectly smooth surface is small. Most of the slope inclinations are concentrated around the horizontal within $30^{\circ}(0.5$ in fig. 3.3). This is true of most surfaces and this fact is often used in simplifying mathematical formulations of scattering.

In section 3.8, we shall refer to the "width of the slope distribution ." By this, we mean the half range of the distribution within which the slopes are contained. For the data in fig. 3.3 this is $30^{\circ}$.

\section{Bulk Inhomogeneities}

We have been unable to isolate any simple theory of bulk inhomogeneity and in section 3.6, we will assume that the entire bulk can be modeled as a uniform medium in which the inhomogeneities are random uniformly distributed point scatterers.

\subsection{The Forescatter Lobe}

Recall from figure 3.1 that the forescatter lobe is spread around the specular direction. There is a considerable amount of published data on the behavior of this lobe [8] [54] [52] [53] [55] [26] [37]. Figure 3.4 [55] is representative of the available data. The figure shows normalized BRDF curves for a rough surface. The BRDF is plotted as a function of $\theta_{r}$ with $\theta_{i}$ and optical roughness as parameters. From this figure and other data, the following conclusions can be drawn:

1. Forescatter is a rough surface phenomenon and independent of the bulk inhomogeneities [8].

2. The forescatter lobe has a specular part and a diffuse part. The specular part is the sharp triangular peak in the BRDF curves of fig. 3.4 (e.g. $\frac{\sigma}{\lambda}=0.63$, $\left.\theta_{i}=75^{\circ}\right)$.

3. Dielectric surfaces as well as metallic surfaces show forescatter [52].

4. Shadowing effects are observable at grazing angles. The curves in fig. 3.4 show a very sharp cutoff at grazing angles. This is due to bi-static shadowing and is explained below.

The forescatter lobe can be explained by geometric and field theories of reflection. The geometric theories provide the best insight into the situation, and we will briefly look at a qualitative explanation of forescatter based on a geometric theory before we present mathematical details of the BRDF for the forescatter lobe. 

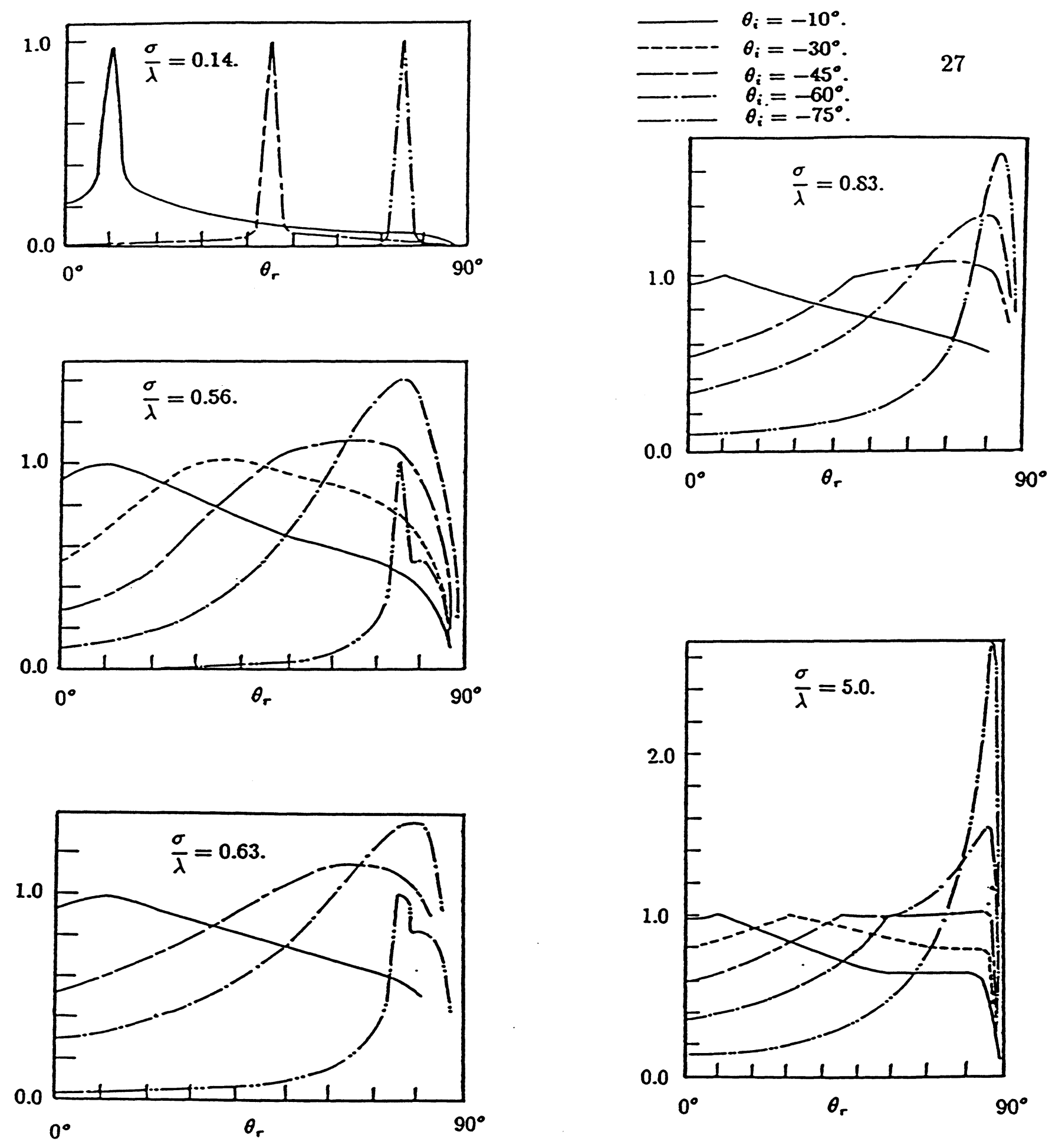

Figure 3.4 Normalized BRDF $\frac{f_{r}\left(\theta_{r}, \pi ; \theta_{i}\right)}{f_{r}\left(\theta_{i}, \pi, \theta_{i}\right)}$ for a rough surface. Adapted from [53]. 


\section{Origin of the Forescatter Lobe}

The forescatter lobe arises from reflection of the incident ray from facets of the rough surface. A facet is a locally plane approximation to the surface. The situation is shown in figure 3.5. Rays are incident on the inclined facets and specularly reflected from them. Since the facets are randomly inclined, the rays are reflected in random directions around the specular direction and give rise to the forescatter lobe.

Figure 3.5 also illustrates how multiple reflection and bi-static shadowing occur in rough surface reflection. The ray A is multiply reflected. Most rough surface scattering theories neglect multiple reflection. Consider the portion the surface marked $\mathrm{S}$ in the figure. Because of the oblique incidence, this area lies in shadow, and of the net area of the surface only a fraction is available for reflection. This reduces the reflectance and the decrease in reflectance is said to be caused by shadowing.

Consider the receiver in the position as shown. The ray $\mathrm{C}$, which would normally have reached the receiver, is multiply reflected away. The area $\mathrm{O}$ in the figure is in effect being occluded from the receiver. Occlusion also reduces the net received flux. The combined effect of shadowing and occlusion is called bi-static shadowing.

Figure 3.5 also illustrates some of the terminology that we will use. The gross angle of incidence, $\theta_{i}$, is the angle that the incident rays make with the mean surface normal. The local angle of incidence, $\psi^{\prime}$, is the angle between the incident ray and the local normal. The local normal is tilted at an angle $\psi$ because the surface is rough.

\section{Theories of Forescatter}

Forescatter has been researched extensively and we will briefly consider three prominent theories:

1. Rice's Theory: Rice [44] developed a small perturbation theory for reflection from rough surfaces. His theory yields a solution if the optical roughness of the surface is much less than 1.0. This is too limiting for our use.

2. Beckmann's Theory: Beckmann and Spizzichino [6] used the physical optics approximation. They start from the Helmholtz integral for computing the reflected field from a surface and apply the Kirchoff approximation to it. The Kirchoff approximation requires that at every point the surface be locally approximated by a plane i.e. the local curvature of the surface is assumed to be zero. Their other assumptions are: 


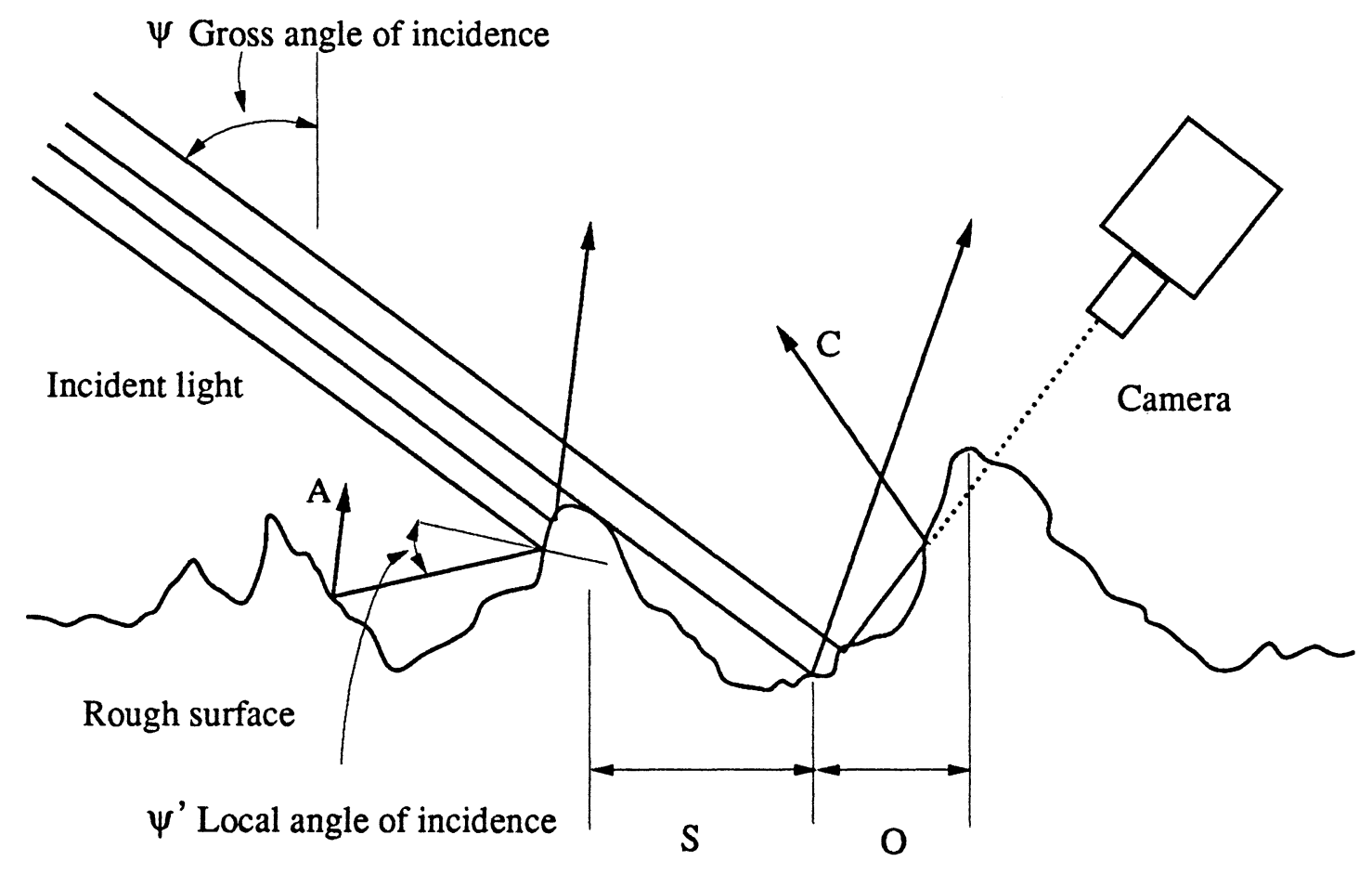

Figure 3.5 Origin of the forescatter lobe 
(a) The surface is perfectly reflecting;

(b) The incident wave is linearly polarized, but the polarization effects of the reflected wave are neglected;

(c) The surface is isotropic and Gaussian.

The Beckmann-Spizzichino expression for the BRDF due to forescatter is

$$
f_{r}\left(\theta_{i} ; \theta_{r}, \phi_{r}\right)=f_{s p e c}\left(\theta_{i} ; \theta_{r}, \phi_{r}\right)+f_{\text {diff }}\left(\theta_{i} ; \theta_{r}, \phi_{r}\right),
$$

where,

$$
\begin{aligned}
f_{\text {spec }}\left(\theta_{i} ; \theta_{r}, \phi_{r}\right) & =\text { Specular part of the lobe } \\
& =\frac{\exp \left[-\left(\frac{4 \pi \cos \theta_{i} \sigma}{\lambda}\right)^{2}\right]}{\cos \theta_{i}} \delta\left(\theta_{r}-\theta_{i}\right) \delta\left(\phi_{r}-\pi\right) .
\end{aligned}
$$

And,

$$
\begin{aligned}
f_{\text {diff }}\left(\theta_{i} ; \theta_{r}, \phi_{r}\right) & =\text { Diffuse part of the lobe } \\
& =\frac{\pi B(\rho / \lambda)^{2}}{\cos \theta_{r} \cos \theta_{i}} \exp \left[-\left(\frac{\sigma}{\lambda} G\right)^{2}\right] \sum_{m=1}^{\infty} \frac{(G \sigma / \lambda)^{2 m}}{m !} \exp \left[-\frac{\pi^{2}}{m}\left\{\frac{\rho}{\lambda}\right\}^{2} H\right]
\end{aligned}
$$

where,

$$
\begin{aligned}
B\left(\theta_{i} ; \theta_{r}, \phi_{r}\right) & =\left[\frac{1+\cos \theta_{i} \cos \theta_{r}+\sin \theta_{i} \sin \theta_{r} \cos \phi_{r}}{\cos \theta_{i} \cos \theta_{r}}\right]^{2} \\
H\left(\theta_{i} ; \theta_{r}, \phi_{r}\right) & =\sin ^{2} \theta_{i}+\sin ^{2} \theta_{r}+2 \sin \theta_{i} \sin \theta_{r} \cos \phi_{r} \\
G\left(\theta_{i} ; \theta_{r}, \phi_{r}\right) & =2 \pi\left(\cos \theta_{i}+\cos \theta_{r}\right) .
\end{aligned}
$$

And, $\sigma$ and $\rho$ are the variance of the height distribution and the correlation length as defined in equations (3.4) and (3.5).

The above expression is complex and Beckmann and Spizzichino offer approximations for small and large optical roughnesses. Houchens and Herring [26] have found that the Beckmann and Spizzichino theory fits experimental data very well. 
3. Torrance-Sparrow Theory: The Torrance-Sparrow theory [54] is based on geometric optics. The surface facet angles are assumed to have a Gaussian distribution as in equation (3.6). Given the incident and reflection angles, only those facets which can reflect specularly from the incident direction into the reflected direction are assumed to contribute to the flux. Multiple reflection is not taken into account.

If we neglect bi-static shadowing, the Torrance and Sparrow expression for the BRDF can be written as :

$$
f_{\text {diff }}\left(\theta_{i} ; \theta_{r}, \phi_{r}\right)=\frac{F\left(\psi^{\prime}, \eta\right) \exp \left(-\psi^{2} c^{2}\right)}{\cos \theta_{i} \cos \theta_{r}}
$$

where, $\eta$ is the complex index of refraction of the surface, and $F()$ the Fresnel reflectivity. $c$ is a constant depending on the optical roughness of the material. $\psi^{\prime}$ is the local angle of incidence and $\psi$ is the inclination of the reflecting facet normal to the surface normal.

$$
\begin{aligned}
\psi^{\prime} & =\text { The local angle of incidence, } \\
& =\frac{1}{2} \cos ^{-1}\left[\cos \theta_{i} \cos \theta_{r}+\sin \theta_{i} \sin \theta_{r} \cos \phi_{r}\right], \\
\psi & =\cos ^{-1}\left[\cos \theta_{i} \cos \psi^{\prime}+\sin \theta_{i} \sin \psi^{\prime} \cos \beta_{1}\right], \\
\beta_{1} & =\sin ^{-1}\left[\sin \phi_{r} \sin \theta_{r} / \sin 2 \psi^{\prime}\right] .
\end{aligned}
$$

The angle $\psi$, has the distribution of equation (3.6). In fact, the exponential term in (3.7) comes directly from equation (3.6), and assuming a different distribution would change only the exponential term in the formula.

Torrance and Sparrow have conducted extensive experiments to validate their formula for metallic and dielectric surfaces and the reader is referred to [54] for details. The assumption of geometric optics and single reflection is substantiated by Rense's study [43] of the polarization of light reflected from rough surfaces. Rense reported good agreement between the single reflection hypothesis and experimental data up to angles of incidence of $45^{\circ}$.

We will use the Torrance-Sparrow theory in our formulation.

In terms of unit normal vectors, the above expression can be written as

$$
f_{\text {diff }}(\mathbf{i}, \mathbf{n}, \mathbf{r})=\frac{F\left(\cos ^{-1} \mathbf{n}_{\mathbf{s}}{ }^{T} \mathbf{i}, \eta\right) \exp \left(-\left(\cos ^{-1} \mathbf{n}_{\mathbf{s}}{ }^{T} \mathbf{n}\right)^{2} c^{2}\right)}{\left(\mathbf{i}^{T} \mathbf{n}\right)\left(\mathbf{r}^{T} \mathbf{n}\right)}
$$


where, $\mathbf{n}_{\mathbf{s}}$ is the specular direction given by $(2.10)$.

Equation (3.8) can be generalized by assuming that the distribution of surface slope angles is given by some general distribution $p\left(\mathbf{n}_{\mathbf{s}}{ }^{T} \mathbf{n}\right)$ instead of the Gaussian. Equation (3.8) now becomes

$$
f_{\text {diff }}(\mathbf{i}, \mathbf{n}, \mathbf{r})=\frac{F\left(\cos ^{-1} \mathbf{n}_{\mathrm{s}}^{T} \mathbf{i}, \eta\right) p\left(\mathbf{n}_{\mathbf{s}}^{T} \mathbf{n}\right)}{\left(\mathbf{i}^{T} \mathbf{n}\right)\left(\mathbf{r}^{T} \mathbf{n}\right)}
$$

So far we have neglected bi-static shadowing. Let us now consider how bi-static shadowing modifies the Torrance-Sparrow BRDF. Beckmann first proposed [5] that the simplest way to take bi-static shadowing into account is to multiply the BRDF by a bi-static shadowing function $S\left(\theta_{i} ; \theta_{r}, \phi_{r}\right)$. The function $S()$ has a range from 0 to 1 and is the fraction of the area of the surface that is neither shadowed nor occluded. Subsequent to Beckmann's theory, other theories of shadowing have also been proposed [48] [58]. One alternative is the bi-static shadowing function $R\left(\theta_{i} ; \theta_{r}, \phi_{r}\right)$ [12]. $R()$ also has a range from 0 to 1 , but represents the percentage of slopes on the surface that are oriented in the desired direction which are neither shadowed nor occluded. Brockelman and Hagofers have argued [12] that $R()$ is a more appropriate function to use than $S()$. However, $R()$ is difficult to evaluate analytically and we will use $S($ ) here.

We will use the shadowing function proposed by Smith [48]. Smith's paper provides us with only the shadowing function. Wagner [58] shows how to calculate the bi-static shadowing function from the shadowing function. If we use Smith's shadowing function in Wagner's theory we get the following bi-static shadowing function:.

$$
\begin{aligned}
S\left(\theta_{i} ; \theta_{r}, \phi_{r}\right) & =\frac{1}{2} \frac{\operatorname{erf}\left(\mu_{i} / \sqrt{2} \tan \beta_{o}\right)+e r f\left(\mu_{r} / \sqrt{2} \tan \beta_{o}\right)}{1+A\left(\mu_{i}\right)+A\left(\mu_{r}\right)} \quad \ldots \text { for } 0<\theta_{i}<\pi / 2,0<\theta_{r}<\pi / 2 . \\
& =\frac{1-1 / 2 \operatorname{erf}\left(\mu_{i} / \sqrt{2} \tan \beta_{o}\right)}{1+A\left(\mu_{i}\right)} \ldots \text { for } 0<\theta_{i}<\pi / 2,-\theta_{i}<\theta_{r}<0, \frac{\pi}{2} \phi_{r}<\pi \\
& =\frac{1-1 / 2 \operatorname{erf}\left(\left\|\mu_{r}\right\| / \sqrt{2} \tan \beta_{o}\right)}{1+A\left(\left\|\mu_{r}\right\|\right)} \ldots 0<\theta_{i}<\pi / 2,-\pi / 2<\theta_{r}<-\theta_{i}, \frac{\pi}{2}<\phi_{r}<\pi
\end{aligned}
$$

where,

$$
\begin{aligned}
& \mu_{1}=\cot \theta_{i}, \\
& \mu_{2}=\cot \theta_{r},
\end{aligned}
$$




$$
A(\mu)=\frac{1}{2}\left\{\sqrt{\frac{2}{\pi}} \frac{\beta_{o}}{\mu} e^{-\mu^{2} / 2 \tan ^{2} \beta_{o}}-\operatorname{erfc}\left(\mu / \sqrt{2} \tan \beta_{o}\right)\right\} .
$$

Putting together the Torrance-Sparrow theory with bi-static shadowing we get

$$
f_{s p e c}(\mathbf{i}, \mathbf{n}, \mathbf{r})+f_{f s c}(\mathbf{i}, \mathbf{n}, \mathbf{r})=f_{s p e c}(\mathbf{i}, \mathbf{n}, \mathbf{r})+\rho_{f s c}\left[S(\mathbf{i}, \mathbf{n}, \mathbf{r}) f_{d i f f}(\mathbf{n}, \mathbf{i}, \mathbf{r})\right]
$$

where, $\rho_{f s c}$ is the forescatter albedo, $S()$ is the bi-static shadowing function in equation $(3.10), f_{\text {diff }}()$ is given by equation (3.9). $f_{\text {spec }}()$ is the specular term:

$$
f_{\text {spec }}(\mathbf{i}, \mathbf{n}, \mathbf{r})=\zeta \delta\left(1-\mathbf{n} \cdot \mathbf{n}_{\mathbf{s}}\right) \text {. }
$$

The Gaussian is a useful expression for the $p()$ in equation (3.11) and if this is used, equation (3.6) should be substituted in it.

\subsection{The Normal Lobe}

A considerable fraction of reflected light from real world surfaces is symmetric about the surface normal. This is the normal lobe of the BRDF. All of the reflected light in this lobe is diffuse. A survey of the experimental data in the literature shows that the normal lobe is very close to being Lambertian. There is extensive data in [35] concerning the validity of Lambert's law. Kortum [35] concludes that Lambert's law is strictly valid for angles of incidence less than $40^{\circ}$ and angles of reflection less than $80^{\circ}$ and is approximately valid for almost the entire range of incident and reflection angles.

Any theory of the normal lobe should explain Lambert's law. Unfortunately, a complete explanation of Lambert's law is still not available. However, there are theories in the literature that come close to doing this and in this section we will review some of them. The explanation of Lambert's law that we propose is based on Chandrasekhar's radiative transfer theory. We will take the main results of that theory and qualitatively demonstrate how Lambert's law comes about.

Perhaps the most remarkable fact about Lambert's law is that it is valid for a large range of materials and seems to be independent of the type of the reflecting material. One of the main achievements of radiative transfer theory is an explanation of the independence of the law with respect to the object material. 


\section{Theories about Lambert's law}

\section{Surface Models}

Bouguer made the first attempts to explain Lambert's Law based on reflection from rough surface facets. Subsequent research established that these models are unable to explain the normal lobe. The reader is referred to [35] for details. Recently, theoretical interest in surface models has resurfaced. This theory, [20] [31] [41], models rough surfaces as random phase screens. The theory is still being developed [1] [2] and a complete explanation of Lambert's law based on it is as yet unavailable.

\section{Bulk Scatter Models}

By far, bulk scatter theories have been more successful in explaining Lambert's law. Bulk scatter theories assume that some of the incident radiation penetrates beneath the surface. Inside the bulk of the matter, this radiation encounters inhomogeneities and is repeatedly scattered by them. A part of the scattered radiation eventually finds its way to the surface and is emitted out as the normal lobe. Early attempts to model bulk scattering were made by Seeliger (see [35] for details). Seeliger's model consisted of tiny volume scattering elements each of which received the incident radiation. Incident radiation from neighboring volume elements was neglected. The BRDF given by Seeliger's model is :

$$
f_{r}\left(\theta_{i} ; \theta_{r}, \phi_{r}\right)=\frac{1}{\cos \theta_{i}+\cos \theta_{r}} .
$$

Kortum [35] notes that Seeliger's model does not fit experimental data as well as Lambert's law does.

Subsequent to Seeliger's attempt, the problem of bulk scattering was formulated as Radiative Transfer Theory [14] [46]. We will not get into the details of radiative transfer theory as that would take us afield from our interest. We will state the main assumptions of the theory and present some relevant results from Chandrasekhar [14].

Radiative transfer theory can be explained as follows. Incident light penetrates into the bulk material, and as the incident ray progresses, it is multiply scattered. The medium is not optically dense, so that each scattering is assumed to be due to a single point scatterer. The incident rays are inclined at an arbitrary angle $\theta_{i}$. The radiation emitted at a distance $z$ inside the semi-infinite bulk in any direction $\theta_{r}, \phi_{r}$ is given as a solution to an integro-differential equation. At $z=0$ the solution 
gives the amount of reflected radiation. Chandrasekhar [14] obtained the complete solution for a general phase function $p(\cos \Theta)$. The solution is very complex and instead of presenting the general solution, we report the solutions calculated by him for isotropic and Rayleigh scattering. These give a better insight into the effect of multiple scattering.

For isotropic scatterers, the BRDF for bulk scattering is given by:

$$
f_{r}\left(\theta_{i} ; \theta_{r}, \phi_{r}\right)=\frac{1}{4} \frac{w_{o}}{u_{i}+u_{r}} H\left(u_{i}\right) H\left(u_{r}\right)
$$

where,

$$
\begin{aligned}
& u_{i}=\cos \theta_{i}, \\
& u_{r}=\cos \theta_{r} .
\end{aligned}
$$

And,

$$
H(u)=\frac{1}{\mu_{1} \cdots \mu_{n}} \frac{\prod_{i=1}^{n} \mu+u_{i}}{\prod_{j}\left(1+k_{j} u\right)}
$$

Here, $\mu_{i}$ 's are the non-negative zeros of the Legendre polynomial $P_{2 n}(\mu)$ of order $2 \mathrm{n}$. $k_{j}$ 's are the non-negative roots of the equation

$$
1=2 \sum_{l=1}^{n} \frac{a_{l} \Psi\left(\mu_{l}\right)}{1-k^{2} \mu_{l}^{2}} \text {. }
$$

$\Psi(\mu)$ is called the characteristic polynomial and for the isotropic case it is

$$
\Psi(\mu)=\frac{1}{2} \omega_{\circ}
$$

The constants $a_{l}$ are given by

$$
a_{l}=\frac{1}{P_{n}^{\prime}\left(\mu_{l}\right)} \int_{-1}^{1} \frac{P_{n}(\mu)}{\mu-\mu_{l}} d \mu .
$$

$\omega_{o}$ is the constant in (4.1). Strictly speaking, the above solution is valid only when $n$ is infinity. However, truncating $\mathrm{n}$ to a small finite number gives a very good approximation.

We will not reproduce the analytical form of the solution for the Rayleigh scattering profile but show the results graphically. Figure 3.6 shows the reflected ra- 


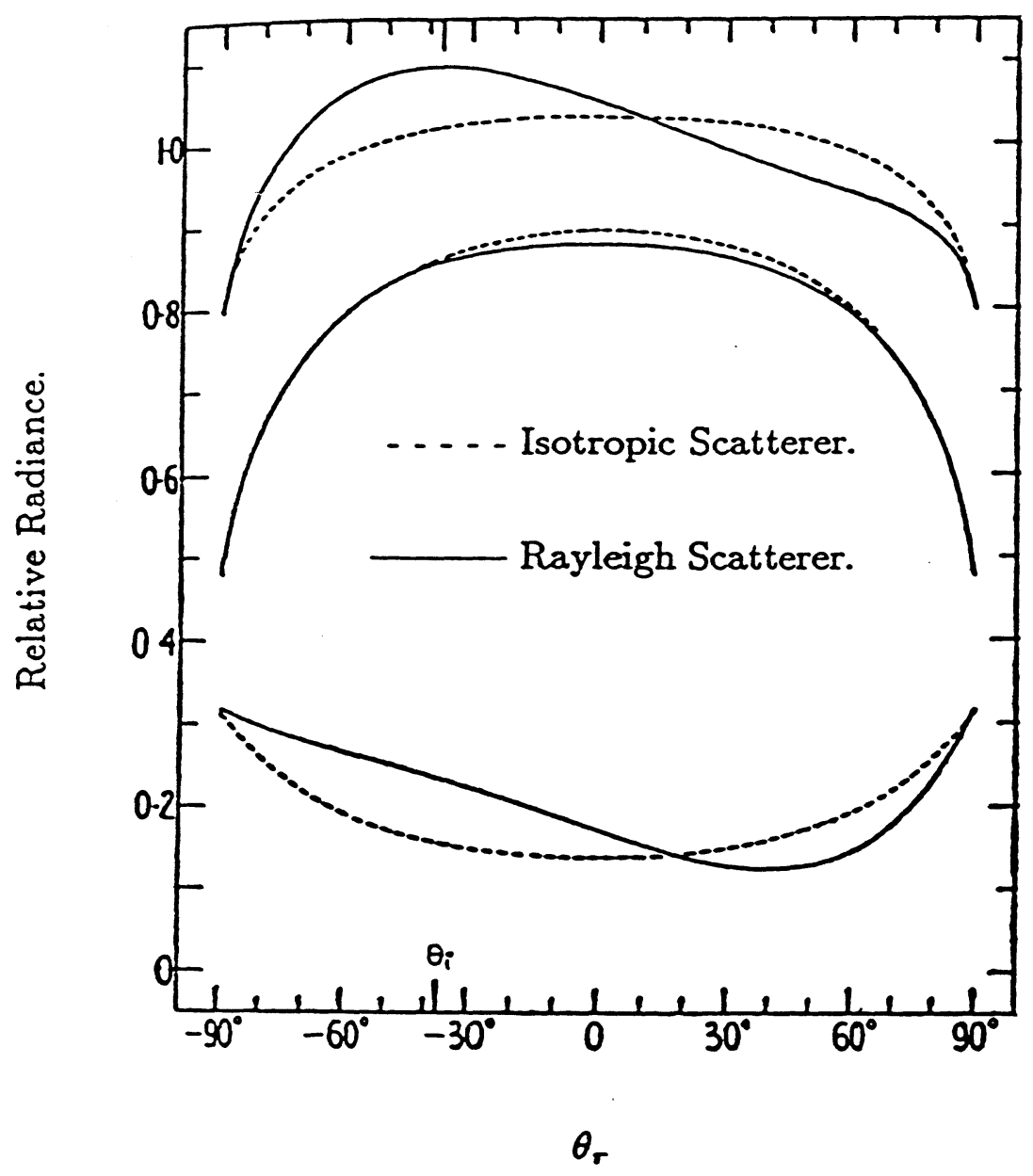

Figure 3.6 Results of the radiative transfer theory 
diance for isotropic and Rayleigh scatterers. The quantity plotted in fig. 3.6 is $f_{r}\left(\theta_{i} ; \theta_{r}, \phi_{r}\right) \cos \theta_{r}$. The incidence angle $\theta_{i}$ is $-37^{\circ}$ and only the principal plane is shown. The top curve shows the exact solutions for the isotropic and Rayleigh scatterers. There is a significant amount of backscatter from the Rayleigh scatterers. The lower most curves are due to singly scattered light. The middle curves are the difference between the two, i.e. these curves represent the distribution of the radiation that has suffered scattering twice or more.

Two interesting facts emerge from fig. 3.6. First, the distribution due to multiple scattering is symmetric about the normal. This distribution is a good approximation to Lambert's law [14]. Second, this distribution is independent of the actual scattering profile of the point scatterer - the Rayleigh multiple scattering distribution is almost identical to the isotropic multiple scattering distribution. ${ }^{2}$ This observation will lead us to an explanation of the independence of Lambert's law to object material.

Before we proceed to apply the results of radiative transfer theory to solid objects, one question needs answering. Radiative transfer theory assumes independent single scattering for each scatterer. This is not a good assumption for the kind of optically dense bulk media we are interested in. How does the scattering behavior change when the independent scattering assumption is violated?

Volume scattering from densely packed scatterers has been studied in the context of radiative transfer from fluidized beds [11] [13] [25] [61] and reflection from paints [24]. The experimental data gathered in these references indicates that the main effect of dependent scattering is to change the albedo. The functional form of the BRDF remains the same. Hence, we can use the results of independent scatter radiative transfer theory to get the $\mathrm{BRDF}$ we need by incorporating an albedo into the BRDF.

\section{A Qualitative Explanation of Lambert's Law}

The normal lobe can be modeled as that part of the BRDF which comes from radiation that has undergone multiple scattering. As an approximation, this distribution is Lambertian.

Recall from fig. 3.6 that multiply scattered radiation seems to be distributed independent of the scatterer phase function. Intuitively, and approximately, this can

${ }^{2}$ This is not strictly true as shown by other plots in [14]. However, as an approximation, this is valid. 
be explained as follows. Consider a medium made of Rayleigh scatterers. Deep within the medium, the incident ray is weak. Most of the energy incident upon a point scatterer (call it $\mathrm{A}$ ) is radiation scattered by neighbors. This radiation does not have an angular dependence as the scatterers are uniformly distributed. Clearly, at A, after scattering once more, the independency from angular variation is preserved. Thus multiple scattering for Rayleigh scatterers gives the same profile as multiple scattering for isotropic scatterers.

For Rayleigh scatterers close to the surface, bulk of the incident energy comes from the incident ray. For them, the scattered radiation will display a Rayleigh profile and exhibit considerable backscatter.

\subsection{The Backscatter Lobe}

There are no significant theories in the literature about the backscatter lobe. In lieu of theories, we present some experimental evidence on its behavior and propose a mathematical expression for its shape.

Figure 3.7 shows data from [10] for reflection from Barium Sulphate paint. There is clear evidence of a strong backscatter lobe. Evidence for this lobe can also be found in the data for Magnesium Oxide coatings, Zinc Oxide paint [10] and the TorranceSparrow data [54].

It is possible to build a simple model for the backscatter lobe shape based on the observation of radiative transfer theory that the backscatter lobe originates from single scattering in the bulk medium. For these surfaces, backscatter is due to single scattering close to the surface. Very small perturbations on the surface which cannot be included in the Kirchoff approximation of section 3.5 will also act like point backscatterers on the surface. We can approximate the situation by saying that the scatterers which give rise to backscatter are contained in a plane that lies parallel to the mean surface boundary. We neglect interference effects but consider bi-static shadowing of the surface scatterers. It is easy to show that the BRDF given by our point scatterer model is

$$
f_{s c a t}\left(\theta_{i} ; \theta_{r}, \phi_{r}\right)=\omega S\left(\theta_{i} ; \theta_{r}, \phi_{r}\right) \frac{p\left(\theta_{i} ; \theta_{r}, \phi_{r}\right)}{\cos \theta_{i} \cos \theta_{r}} .
$$

where, $\omega$ is the scatterer density in the plane. $p\left(\theta_{i} ; \theta_{r}, \phi_{r}\right)$ is the phase function for each scatterer and $S()$ is the bi-static shadowing function. If we knew the distribution of the sizes of the scatterers, the phase function $p()$ could be estimated. However, we 


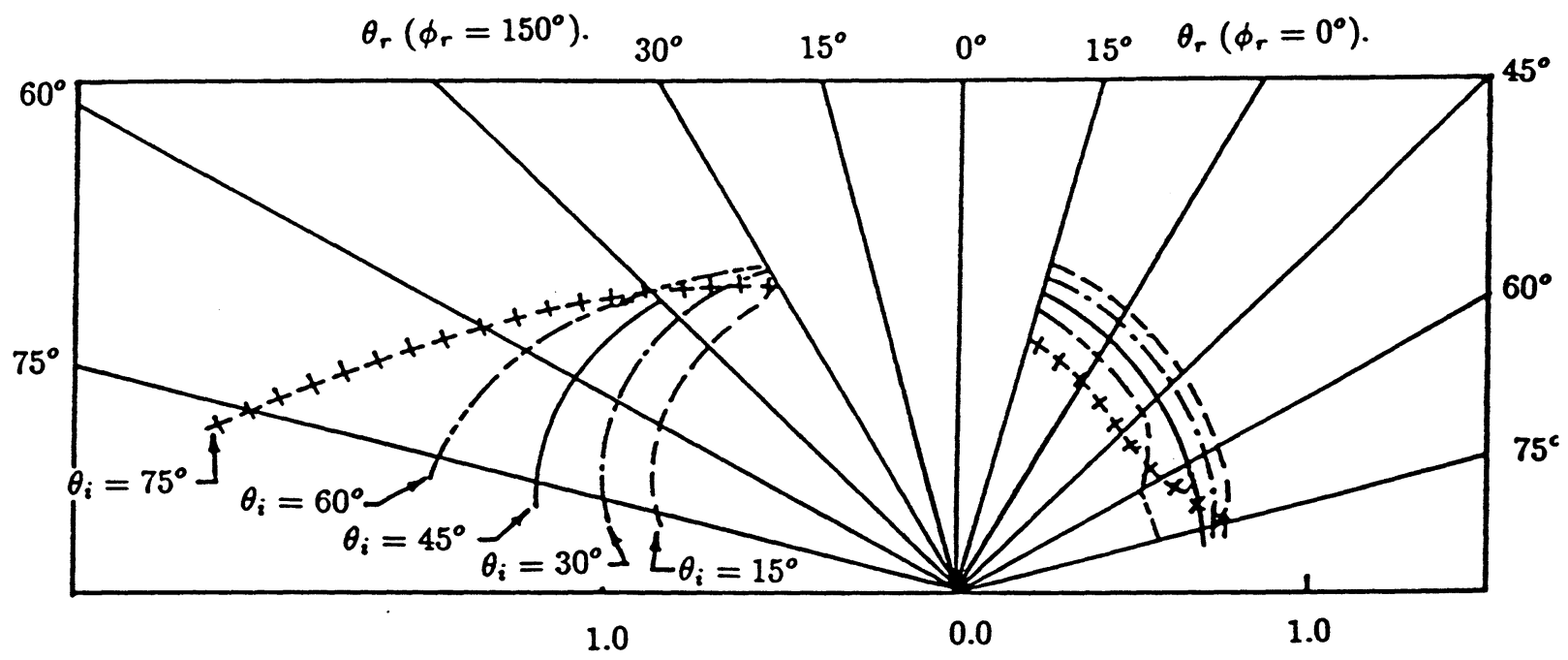

Arbitrarily scaled $f_{r}()$.

Figure 3.7 BRDF showing strong backscatter. Adapted from [10] 
have not been able to find any published literature on this, and are forced to keep its exact form unspecified. We will assume that the phase function depends only on the phase angle.

$f_{\text {scat }}()$ can now be written in terms of unit vectors as:

$$
f_{\text {scat }}(\mathbf{i}, \mathbf{n}, \mathbf{r})=\omega S(\mathbf{i}, \mathbf{n}, \mathbf{r}) \frac{p\left(\mathbf{i}^{T} \mathbf{r}\right)}{\left(\mathbf{i}^{T} \mathbf{n}\right)\left(\mathbf{r}^{T} \mathbf{i}\right)} .
$$

The BRDF due to the backscatter lobe can be written as

$$
\rho_{b s c} f_{b s c}=\rho_{b s c} f_{s c a t}(\mathbf{i}, \mathbf{n}, \mathbf{r})
$$

where, $\rho_{b s c}$ is the backscatter albedo.

\subsection{The Reflectance Map}

We will now proceed to use the BRDF formulae from previous sections to obtain an approximate reflectance map. We will demonstrate the process of approximation. As explained below, the approximations are possible because of the nature of the imaging geometry and are not specific to the BRDF we use.

Putting together equations (3.1), (3.11), (3.15) and using the Lambertian for the normal lobe, we get the net BRDF as

$$
\begin{aligned}
f_{r}(\mathbf{i}, \mathbf{n}, \mathbf{r}) & =\zeta \delta\left(1-\mathbf{n} \cdot \mathbf{n}_{\mathbf{s}}\right) \\
& +\rho_{f s c} S(\mathbf{i}, \mathbf{n}, \mathbf{r}) \frac{F\left(\cos ^{-1}\left(\mathbf{n}_{\mathbf{s}} T_{\mathbf{i}}\right), \eta\right) \exp \left\{-\left[\cos ^{-1}\left(\mathbf{n}_{\mathbf{s}}{ }^{T} \mathbf{n}\right)\right]^{2} c^{2}\right\}}{\left(\mathbf{i}^{T} \mathbf{n}\right)\left(\mathbf{r}^{T} \mathbf{n}\right)} \\
& +\rho_{\text {norm }} \\
& +\rho_{b s c} S(\mathbf{i}, \mathbf{n}, \mathbf{r}) \frac{p\left(\mathbf{i}^{T} \mathbf{r}\right)}{\left(\mathbf{i}^{T} \mathbf{n}\right)\left(\mathbf{r}^{T} \mathbf{n}\right)} .
\end{aligned}
$$

We are not interested in pursuing specular reflection besides noting that it gives an intensity spike at specular orientation. We will drop the specular term.

Dropping the specular term, the exact reflectance map is

$$
\begin{aligned}
R(\mathbf{i}, \mathbf{n}, \mathbf{r}) & =\rho_{f s c} S(\mathbf{i}, \mathbf{n}, \mathbf{r}) \frac{F\left(\cos ^{-1}\left(\mathbf{n}_{\mathbf{s}}{ }^{T} \mathbf{i}\right), \eta\right) \exp \left\{-\left[\cos ^{-1}\left(\mathbf{n}_{\mathbf{s}}{ }^{T} \mathbf{n}\right)\right]^{2} c^{2}\right\}}{\left(\mathbf{r}^{T} \mathbf{n}\right)} \\
& +\rho_{n o r m} \mathbf{i}^{T} \mathbf{n} \\
& +\rho_{b s c} S(\mathbf{i}, \mathbf{n}, \mathbf{r}) \frac{p\left(\mathbf{i}^{T} \mathbf{r}\right)}{\left(\mathbf{r}^{T} \mathbf{n}\right)}
\end{aligned}
$$

There are two features of the geometry of fig. 2.2 that help us approximate equation (3.17): 
1. Recall that the camera and the light source are assumed to be infinitely far away. The vectors $i, r$ and $n_{s}$ are constant over the entire surface.

2. In most situations, the angle that the light source and the camera subtend at the object is usually between $10^{\circ}$ and $45^{\circ}$. The vectors $\mathbf{i}, \mathbf{r}$ and $\mathbf{n}_{\mathbf{s}}$ are close together.

With this in mind, consider the forescatter term. From (1) above, it is clear that $\mathbf{n}_{\mathbf{s}} T_{\mathbf{i}}$ is independent of $\mathbf{n}$. Thus the Fresnel factor $F()$ in the forescatter term is constant and can be absorbed in the factor $\rho_{f s c}$. Next, consider how the bi-static shadowing term affects the forescatter expression. The value of the bi-static shadowing term as a function of $\theta_{n}$ is shown in figure 3.8. The curves are plotted assuming a phase angle of $30^{\circ}$ and are parametrized by the width of the slope distribution of the illuminated surface. Note that for widths around $30^{\circ}$ the bi-static shadowing is prominent when it is close to the ends of the range of $\theta_{n}$. Figure 3.9 shows overlaid plots of the forescatter reflectance map in the principal plane with and without the bi-static shadowing function. The phase angle is $30^{\circ}$, and the surface slope spread for the bi-static shadowing function is assumed to be $50^{\circ}$. The curves are parametrized by the factor $c$ (see equation (3.18)). As $c$ increases, the forescatter lobe width decreases and the effect of the bi-static shadowing function is negligible. Realistic values of $c$ are in the neighborhood of 2.5 [54] and for these, bi-static shadowing does not have a large effect on the reflectance map. It can be approximated by unity.

The forescatter part now becomes

$$
\rho_{f s c} \frac{\exp \left\{-\left[\cos ^{-1}\left(\mathbf{n}_{\mathrm{s}}^{T} \mathbf{n}\right)\right]^{2} c^{2}\right\}}{\left(\mathbf{r}^{T} \mathbf{n}\right)} .
$$

We replace the term $\mathbf{r}^{T} \mathbf{n}$ in the denominator with $\mathbf{n}_{\mathrm{s}}{ }^{T} \mathbf{n}$. The observation (2) above lends support for this. Now, the expression is

$$
\rho_{f s c} \frac{\exp \left\{-\left[\cos ^{-1}\left(\mathbf{n}_{\mathbf{s}}{ }^{T} \mathbf{n}\right)\right]^{2} c^{2}\right\}}{\left(\mathbf{n}_{\mathbf{s}}{ }^{T} \mathbf{n}\right)} \text {. }
$$

The denominator term in this expression becomes significant as $\mathbf{n}_{\mathbf{s}}{ }^{T} \mathbf{n}$ becomes small. However, as this happens, the numerator term decreases and compensates for the increase due to the denominator term. Hence, the denominator dynamics are not effective and we can approximate the denominator term by unity. i.e. we use the expression

$$
\rho_{f s c} \exp \left\{-\left[\cos ^{-1}\left(\mathbf{n}_{\mathbf{s}}^{T} \mathbf{n}\right)\right]^{2} c^{2}\right\}
$$


width of slope distribution (degrees.)

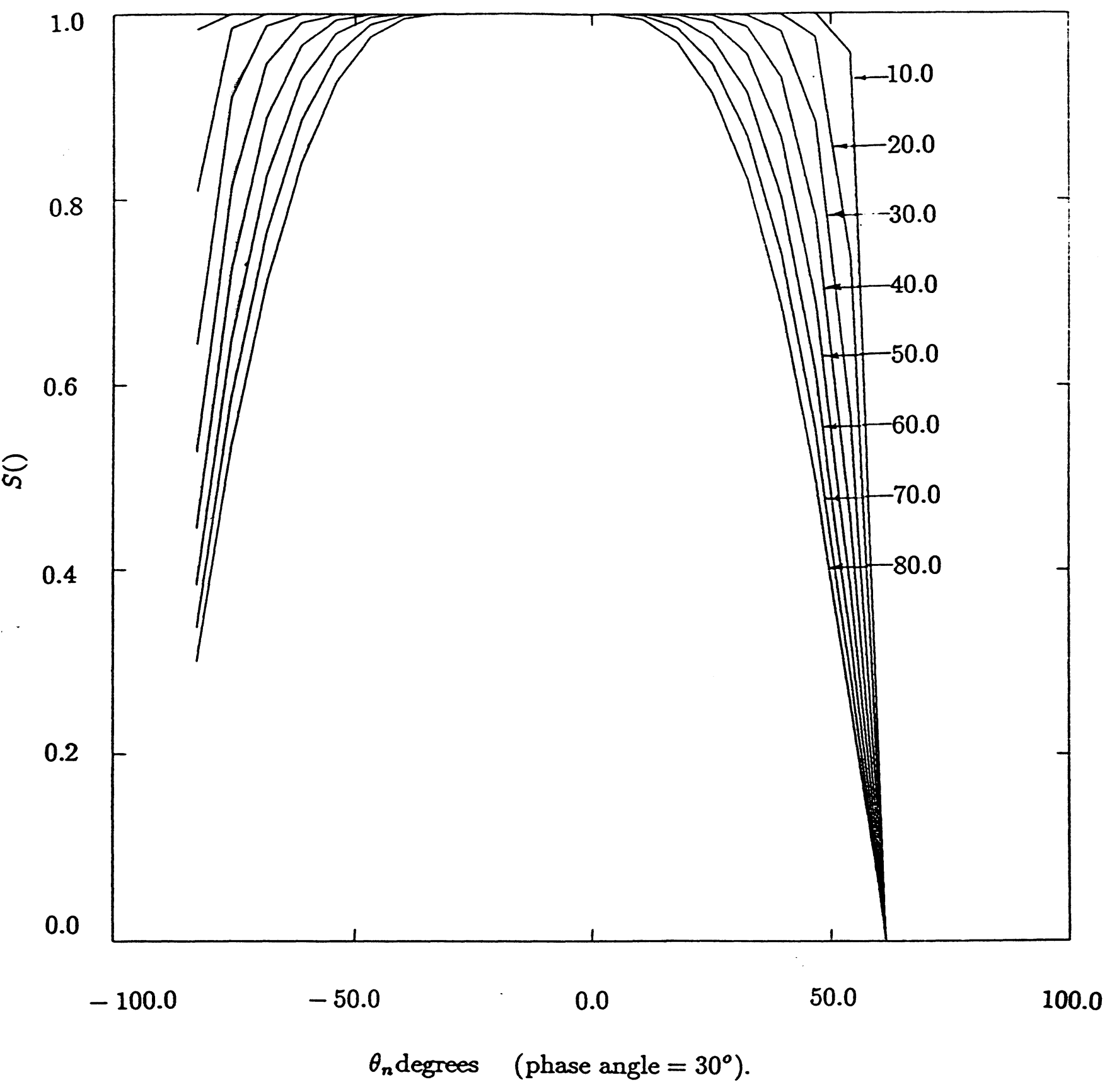

Figure 3.8 Bi-static shadowing as a function of $\theta_{n}$ 


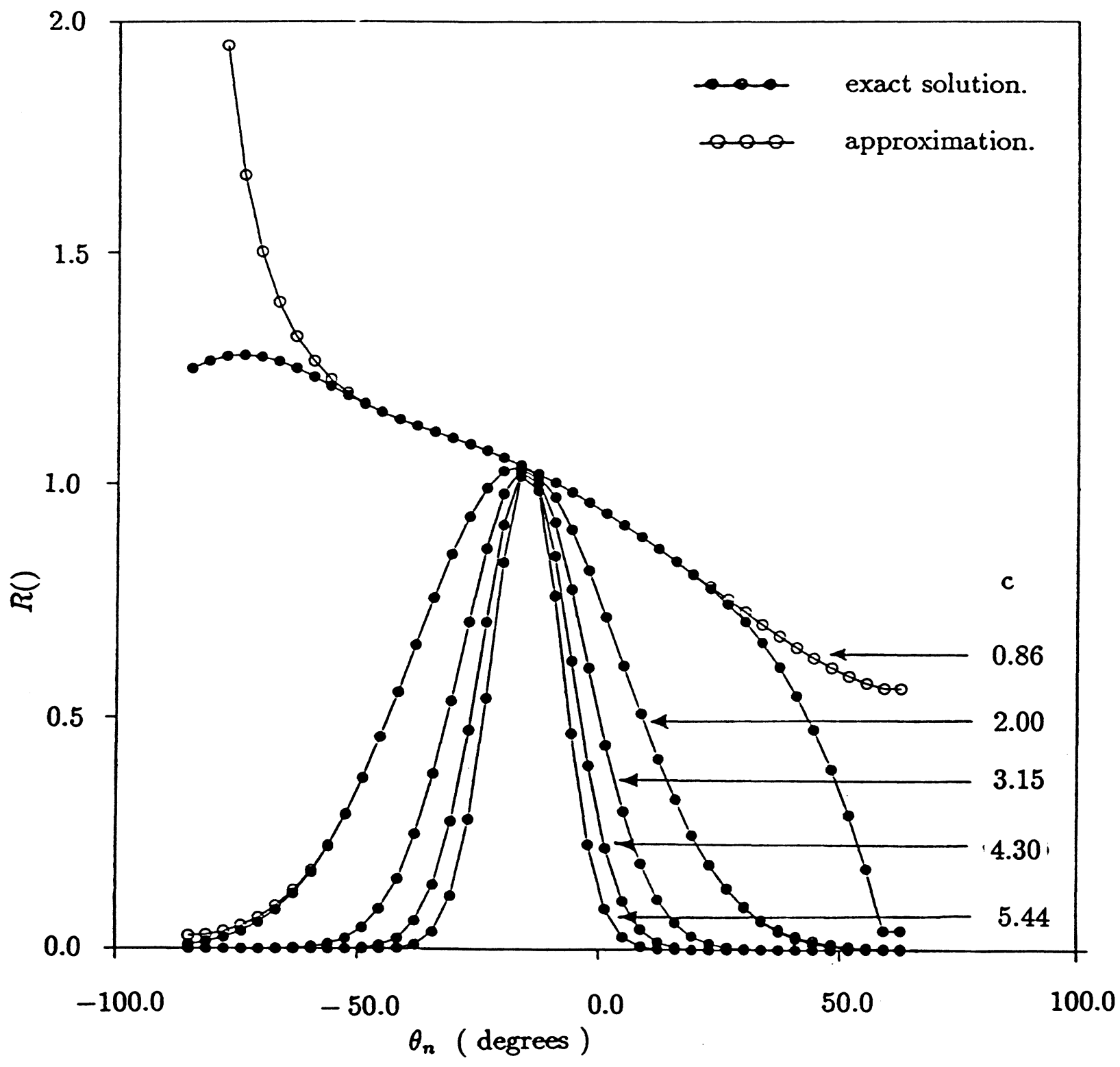

Figure 3.9 Comparison of forescatter reflectance map with and without shadowing 


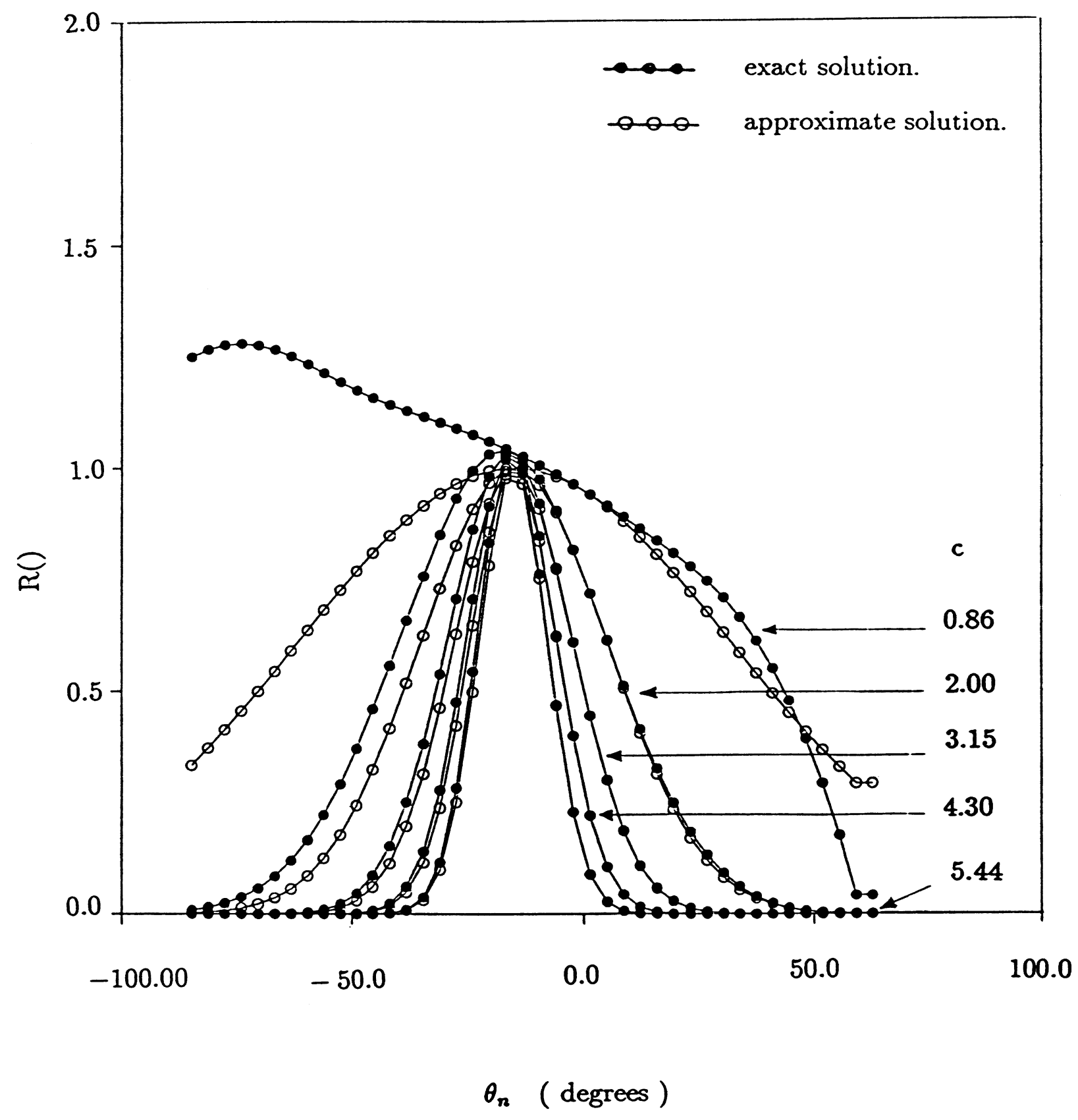

Figure 3.10 Comparison of the exact and approximate forescatter reflectance maps 
Figure 3.10 shows overlayed plots of the exact forescatter term (with bi-static shadowing) and this approximation in the principal plane for a phase angle of $30^{\circ}$. Figure 3.11 shows the contours of the error in the approximation for error less than 10 percent. The error in the approximation was computed as follows.

1. For a given value of $c$ and the phase angle the exact forescatter term was computed for densely sampled values of $\theta_{n}$ and $\phi_{n}$ from the forescatter part of equation (3.17).

2. For the same values of $c$ and phase angle, the approximate forescatter expression (3.18) was used computed at the same points and the maximum absolute difference in the two values over the entire set of $\theta_{n}, \phi_{n}$ points was chosen to be the error.

3. This error was computed for the entire set of $c$ and phase angle values and fig 3.11 is the contour plot of this function.

The plot shows that the approximation is good for a wide range of forescatter lobe widths if the phase angle is less than $30^{\circ}$.

If we use the general forescatter expression (3.9) instead of the Torrance-Sparrow expression (3.8), then, in the spirit of the above argument, the sequence of approximations is valid if $p\left(\mathbf{n}_{\mathbf{s}}{ }^{T} \mathbf{n}\right)$, the slope distribution function, drops off sharply as $\mathbf{n}_{\mathbf{s}}{ }^{T} \mathbf{n}$ decreases. After the approximations, the general forescatter map becomes

$$
\rho_{f s c} p\left(\mathbf{n}_{\mathrm{s}} \cdot \mathbf{n}\right) .
$$

Using different $p\left(\right.$ )'s gives us different forescatter expressions, e.g., using $p\left(\mathbf{n}_{\mathbf{s}}{ }^{T} \mathbf{n}\right)=\left(\mathbf{n}_{\mathbf{s}}{ }^{T} \mathbf{n}\right)^{\gamma}$, $\gamma \geq 0$ gives the forescatter part of Horn's map. Further, this interpretation of the approximate forescatter reflectance map as the scaled slope distribution allows us to judge the validity of any proposed forescatter term. We simply ask whether the proposed term can also constitute a feasible slope distribution.

We will keep the normal term as it is.

Next, consider the backscatter term

$$
\rho_{b s c} S(\mathbf{i}, \mathbf{n}, \mathbf{r}) \frac{p\left(\mathbf{i}^{T} \mathbf{r}\right)}{\left(\mathbf{r}^{T} \mathbf{n}\right)}
$$

The term $\mathbf{i}^{T} \mathbf{r}$ is a constant and so $p\left(\mathbf{i}^{T} \mathbf{r}\right)$ can be absorbed into the albedo. Figure 3.12 shows the backscatter term in the principal plane for a phase angle of $30^{\circ}$. Note 


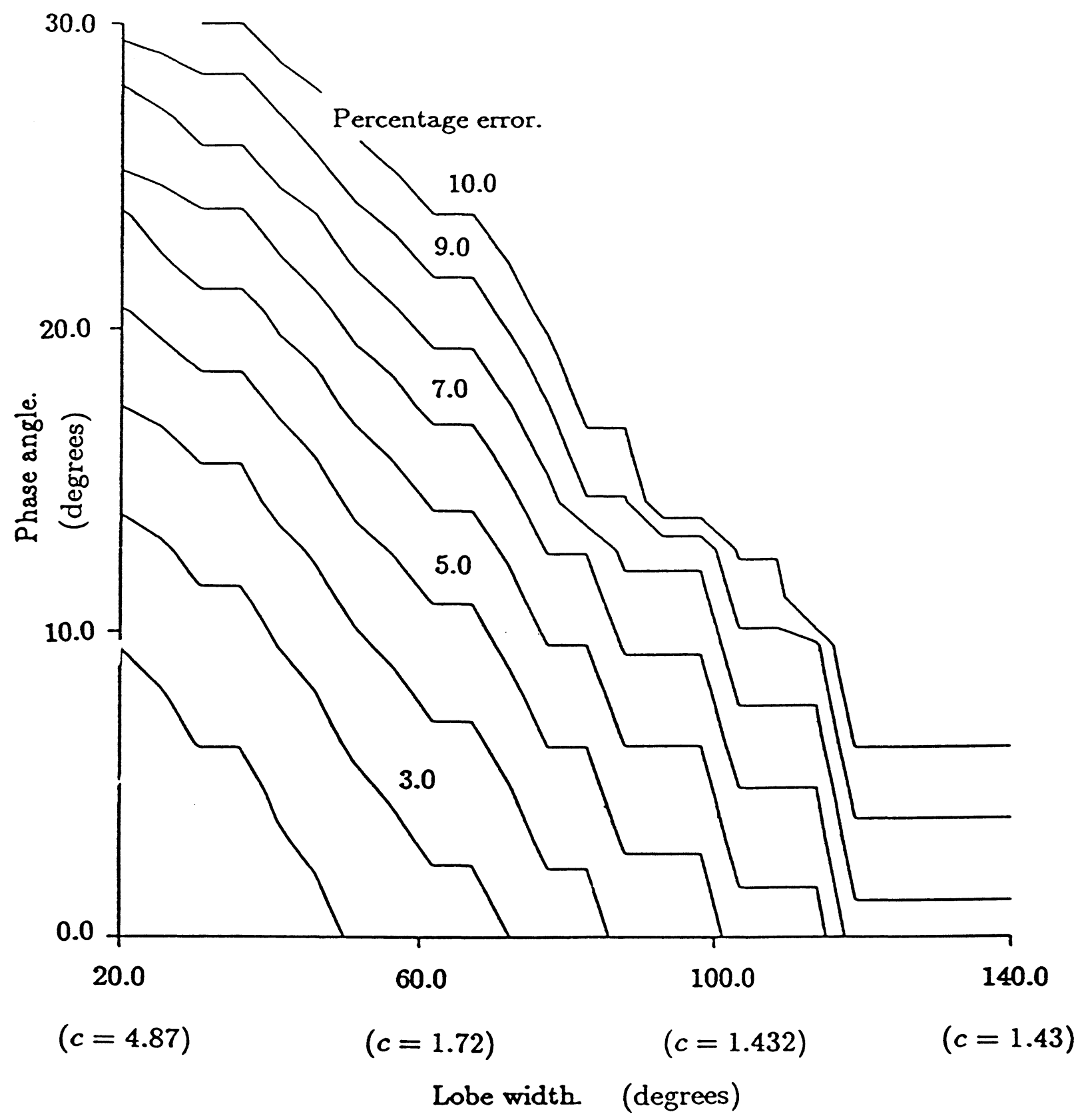

Figure 3.11 Contours of the error in the approximation 


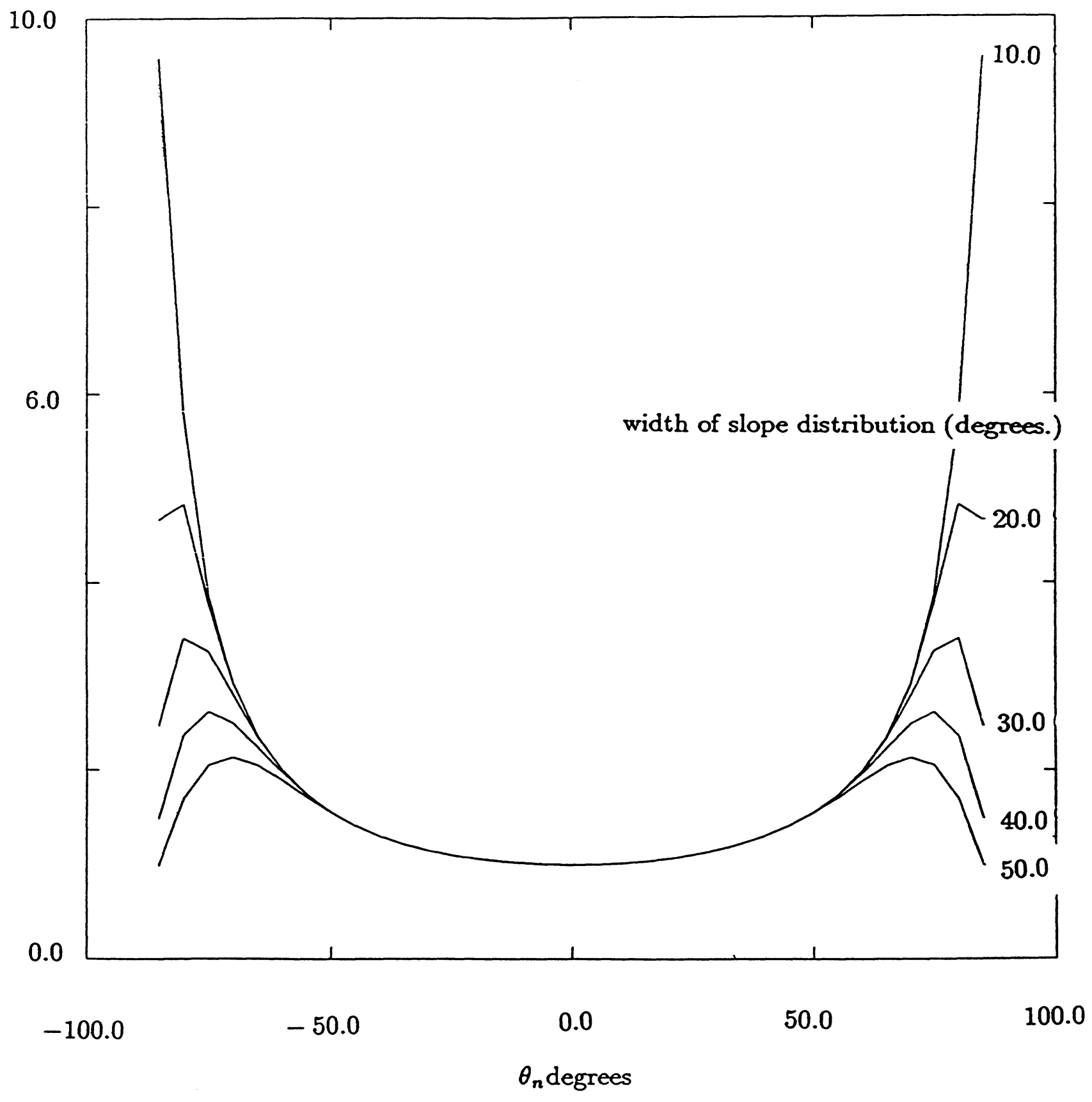

Figure 3.12 The backscatter reflectance map 
that the term is approximately constant over the range of $\theta_{n}$ from $-50^{\circ}$ to $+50^{\circ}$. Outside this range, the term increases briefly, and then decreases as the bi-static shadowing becomes prominent. The increase is caused by the denominator in the above expression.

We will approximate the entire backscatter term by a constant

$\rho_{b s c}$.

In support of our approximation, we note that for most surfaces, backscattering is not very prominent and the above approximation will not cause serious errors.

Using these approximations the resulting reflectance map is

$$
\begin{aligned}
R(\mathbf{n}, \mathbf{i}, \mathbf{r}) & =\rho_{f s c} \exp \left\{-\left[\cos ^{-1}\left(\mathbf{n}_{\mathbf{s}} T^{T} \mathbf{i}\right)\right]^{2} c^{2}\right\} \\
& +\rho_{\text {norm }}\left(\mathbf{n}^{T} \mathbf{i}\right) \\
& +\rho_{b s c} .
\end{aligned}
$$

\subsection{A General Model for Reflectance Maps}

In this section, we will consider a mathematical generalization of equation (3.19). Our aim is to construct a class of reflectance maps that we will call m-lobed reflectance maps. This generalization is based on the following observations:

1. The normal lobe term of equation 3.19 can be written as $\rho_{\text {norm }} I\left(\mathbf{i}^{T} \mathbf{n}\right)$, where, $I()$ is the identity function, $I(x)=x$. The two functions $\exp \left\{-\left[\cos ^{-1}()\right]^{2} c^{2}\right\}$ and $I()$ that occur in equation (3.19) have the following properties: they are monotonic strictly increasing functions of their arguments, they are convex and log-concave.

2. The arguments of the above two functions are inner products of the vector $\mathbf{n}$ with $\mathbf{n}_{\mathbf{s}}$ and $\mathbf{i}$. Both of these vectors are independent of $\mathbf{n}$.

3. Both $\mathbf{n}_{\mathbf{s}}$ and $\mathbf{i}$ lie in the principal plane.

4. For the given viewing direction, the vector $\mathbf{n}_{\mathbf{s}}$ depends solely on $\mathbf{i}$.

We generalize as follows: With each incident direction $\mathbf{i}_{l}$ we associate a set of principal directions $\mathbf{p}_{l j}, j=1, \cdots, m-1$ in the principal plane, i.e. if

$$
\mathbf{i}_{l}=\left[\sin \left(\theta_{l}\right) \cos \left(\phi_{l}\right) \sin \left(\theta_{l}\right) \sin \left(\phi_{l}\right) \cos \left(\theta_{l}\right)\right]^{T},
$$


then

$$
\mathrm{p}_{l j}=\left[\sin \left(\theta_{l j}\right) \cos \left(\phi_{l}\right) \sin \left(\theta_{l j}\right) \sin \left(\phi_{l}\right) \cos \left(\theta_{l j}\right)\right]^{T},
$$

where $0<\theta_{l 1}<\theta_{l 2}<\cdots<\theta_{l m-1}<\frac{\pi}{2}$. See figure 3.13 for an illustration.

With each direction $\mathrm{p}_{l j}$ we associate $\phi_{j}()^{3}$, a monotonic strictly increasing function and $\rho_{j}$, an albedo. We also assume that $\phi_{j}()$ are convex and log-concave. Then,

$$
R\left(\mathbf{i}_{l}, \mathbf{n}, \mathbf{r}\right)=\sum_{j=1}^{m-1} \rho_{j} \phi_{j}\left(\mathbf{p}_{l j}^{T} \mathbf{n}\right)+b,
$$

where, $b \geq 0$ is a constant.

Before we proceed, we remark that the reflectance map proposed in equation (3.21) is very general and includes iso-tropic and non-isotropic maps. For an isotropic reflectance map, if the zenith angles of the light sources $\theta_{l}$ were constant, the zenith angles $\theta_{l j}$ of the principal directions would be independent of the azimuth angles i.e. $\theta_{l_{1} j}=\theta_{l_{2 j} j}$. When this is not true, the reflectance map is non-isotropic.

We will also assume that the term $\sum_{j=1}^{m-1} \rho_{j} \phi_{j}\left(\mathbf{p}_{l j}^{T} \mathbf{n}\right)$ goes to zero as $\mathbf{i}_{l}^{T} \mathbf{n}$ does. Physically, this assumption means that the only lobe that contributes to the observed intensity as we get closer to the self shadow region $\left(\mathbf{i}_{l}^{T} \mathbf{n} \leq 0\right)$ is the backscatter lobe.

Finally, let us agree on some nomenclature for the rest of the thesis. We will refer to the reflectance map model of equation (3.21) as the m-lobed reflectance map. We will not assume any specific form for the $\phi()$ functions and will only assume that they have the properties listed above. The map of equation (3.19) will be referred to as the physical reflectance map and in using it we will assume the explicit form of the $\phi()$ functions of equation (3.19).

\subsection{Experimental Evidence}

As a final demonstration of the utility of the physical reflectance map, we present the intensity values recorded in an image and the fit of this model and a Lambertian model to this data.

Figure 3.14 shows the image of a spherical surface. Figure 3.15 shows the intensity data in the principal plane and a fit of equation (3.19) to the data. The parameters

\footnotetext{
${ }^{3}$ Note the potential confusion between the monotonic functions $\phi()$ and the azimuth angle $\phi$. To prevent this, we will indicate the function with a pair of empty brackets after it, i.e., as $\phi()$, and the angle without them, i.e., as $\phi$.
} 


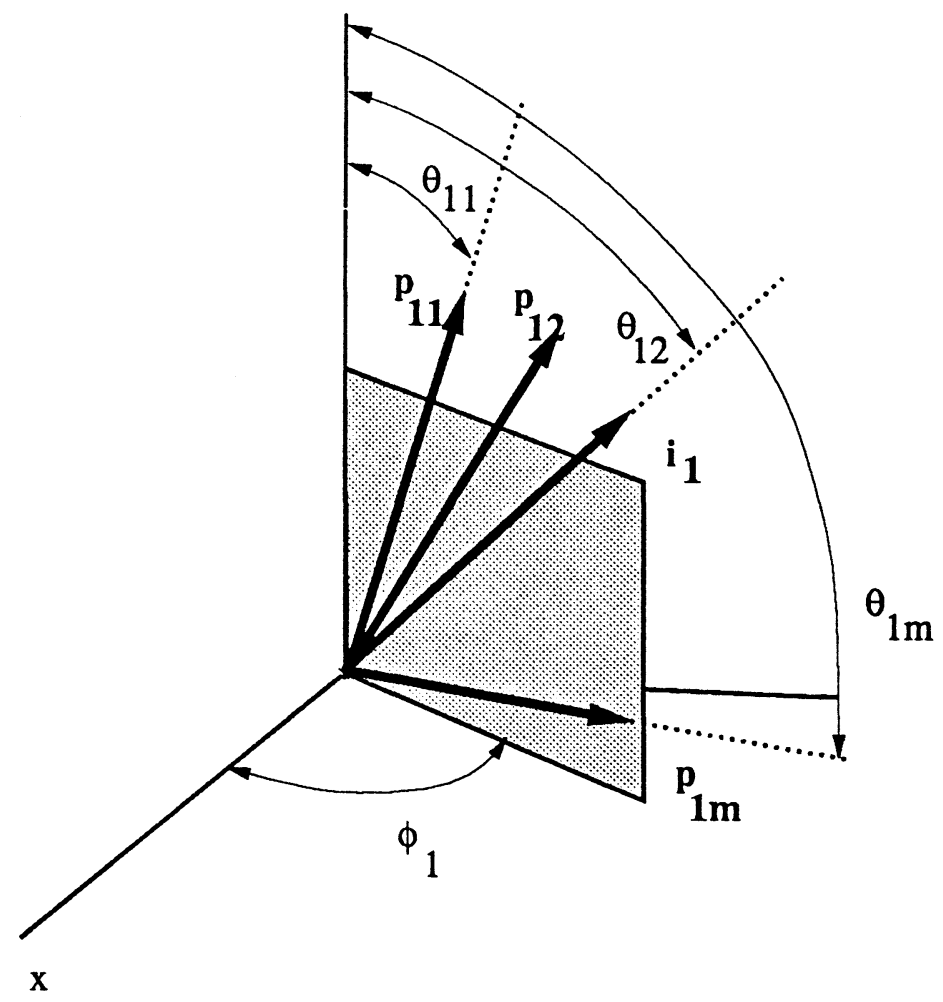

Figure 3.13 Principle directions for the m-lobed map 


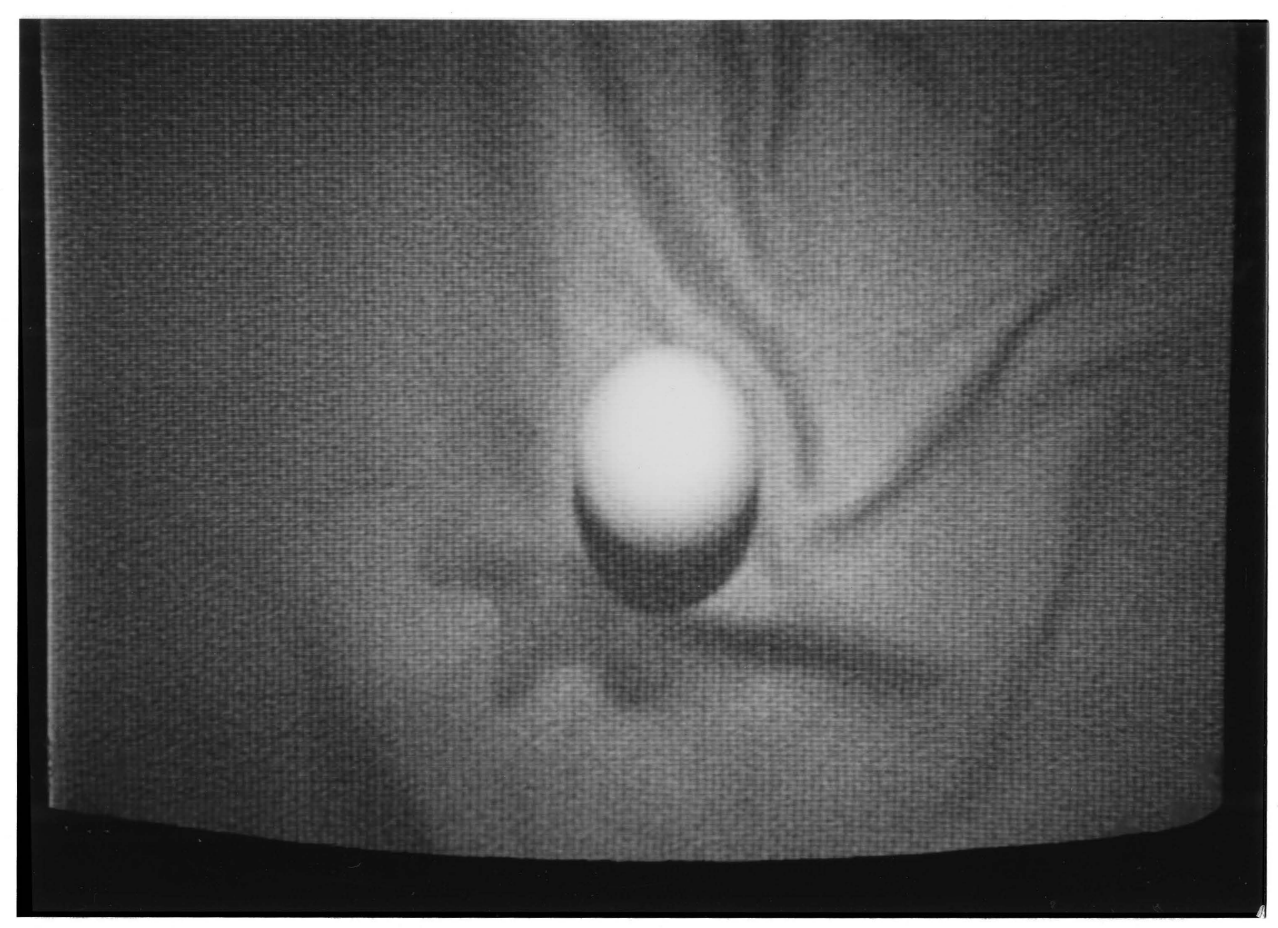

Figure 3.14 A spherical surface

used are

$$
\rho_{f s c}=1.0, \rho_{\text {norm }}=0.5 \rho_{b s c}=0.0 c=2.578 .
$$

The phase angle is $33^{\circ}$. The figure also shows the intensity profile that would result from using only a Lambertian reflectance map. It is clear from the figure that our proposed reflectance map models the intensity pattern more accurately than the Lambertian model. 


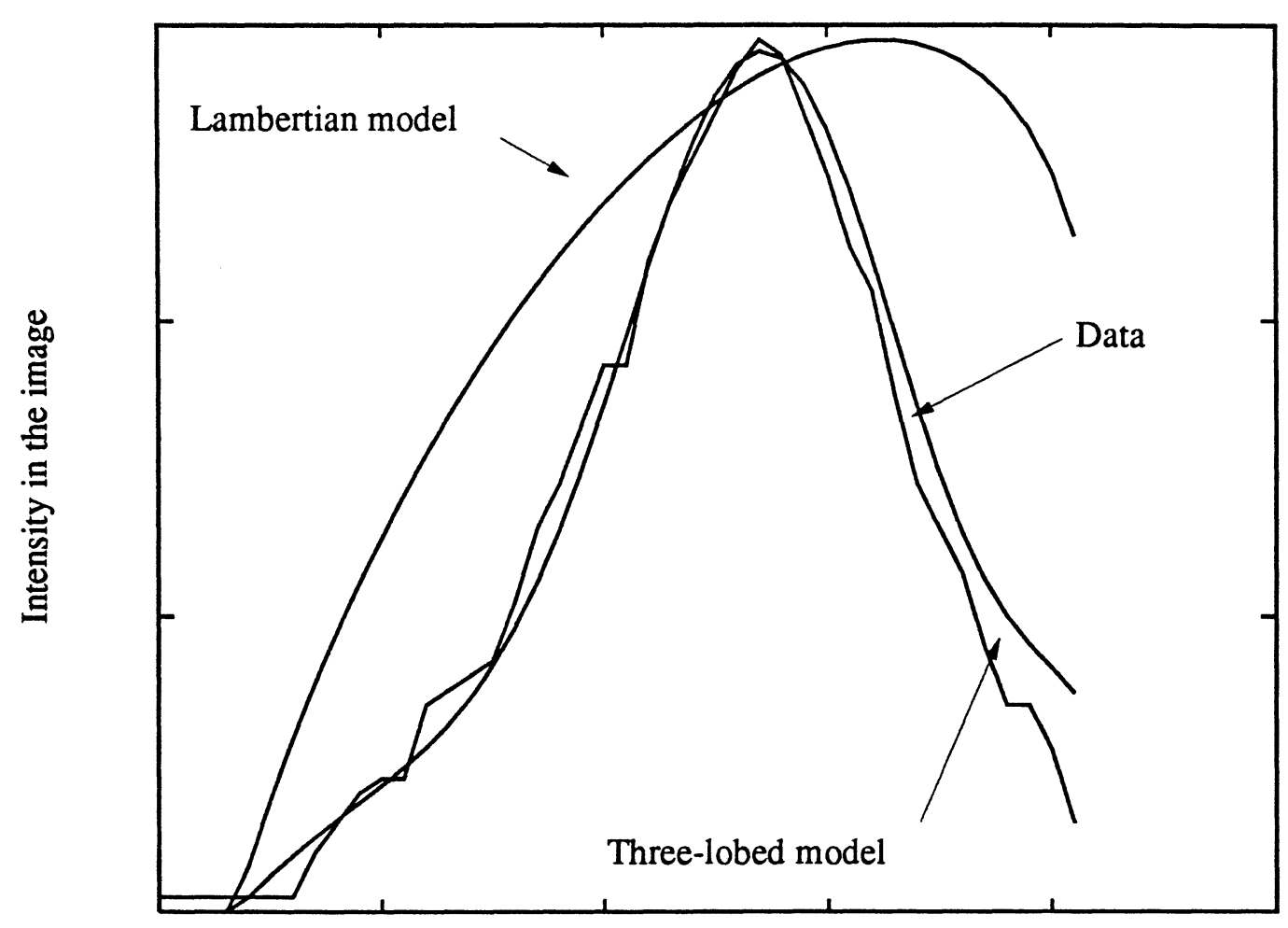

Distance along the principle plane

Figure 3.15 Intensity in the principle plane 


\section{Chapter 4}

\section{The Normalized Photometric Stereo Equation}

\subsection{Introduction}

In this chapter, we will investigate the normalized photometric stereo equation 2.8 . We will use the m-lobed reflectance map of equation 3.21. The main concern of this chapter will be the number of light sources needed to achieve a globally unique solution. i.e. we ask what is the minimum value of $k$, such that any two $\mathbf{n}_{1}, \mathbf{n}_{2}$; $\mathbf{n}_{1} \neq \mathbf{n}_{2}$ imply

$$
\left(\begin{array}{c}
R\left(\mathbf{i}_{1}, \mathbf{n}_{1}, \mathbf{r}\right) \\
\ldots \\
R\left(\mathbf{i}_{k}, \mathbf{n}_{1}, \mathbf{r}\right)
\end{array}\right) \neq\left(\begin{array}{c}
R\left(\mathbf{i}_{1}, \mathbf{n}_{2}, \mathbf{r}\right) \\
\ldots \\
R\left(\mathbf{i}_{k}, \mathbf{n}_{2}, \mathbf{r}\right)
\end{array}\right) \quad ?
$$

Recall that the normalized equation becomes linear if we assume that the surface is Lambertian. Woodham [60] proved that in this case, three sources were necessary and sufficient to obtain a globally unique solution.

When we consider non-Lambertian surfaces we have a non-linear normalized photometric stereo equation. In this chapter, we derive three results about the solution of the normalized photometric stereo equation. The results are expressed in theorems $4.1,4.2$ and 4.3 of this chapter. Informally, the results are

- It is possible to obtain a globally unique solution to the normalized photometric stereo equation in the case of reflectance maps with m-lobes. Theorem 4.1 states the conditions under which this can be done with only three light sources.

- Theorems 4.2 and 4.3 translate the condition of theorem 4.1 into constraints on light source directions. As will become clear, these constraints are usually met with in all photometric stereo reconstructions. 
If we assume $k=3$, we can write the normalized photometric stereo equation 2.8 with the m-lobed map as

$$
\left(\begin{array}{c}
I_{1}^{r e l}(\mathbf{n}) \\
I_{2}^{r e l}(\mathbf{n}) \\
I_{3}^{r e l}(\mathbf{n})
\end{array}\right)=\sum_{j=1}^{m-1} \rho_{j}\left(\begin{array}{l}
\phi_{j}\left(\mathbf{p}_{1 j}^{T} \mathbf{n}\right) \\
\phi_{j}\left(\mathbf{p}_{2 j}^{T} \mathbf{n}\right) \\
\phi_{j}\left(\mathbf{p}_{3 j}^{T} \mathbf{n}\right)
\end{array}\right)+\left(\begin{array}{l}
b \\
b \\
b
\end{array}\right) .
$$

Let us define matrices $H_{j}$ as

$$
H_{j}=\left(\begin{array}{c}
\mathbf{p}_{1 j}^{T} \\
\mathbf{p}_{2 j}^{T} \\
\mathbf{p}_{3 j}^{T}
\end{array}\right)
$$

If we assume that the light source directions $i_{1}, i_{2}, i_{3}$ are linearly independent then it is easy to show that the matrices $H_{j}$ are invertible.

If we assume that there exist $\mathbf{n}_{1}$ and $\mathbf{n}_{2}$, such that $\mathbf{n}_{1} \neq \mathbf{n}_{2}$ and

$$
\left[I_{1}^{\text {rel }}\left(\mathbf{n}_{1}\right) I_{2}^{r e l}\left(\mathbf{n}_{1}\right) I_{3}^{r e l}\left(\mathbf{n}_{1}\right)\right]^{T}=\left[I_{1}^{\text {rel }}\left(\mathbf{n}_{2}\right) I_{2}^{r e l}\left(\mathbf{n}_{2}\right) I_{3}^{r e l}\left(\mathbf{n}_{2}\right)\right]^{T},
$$

then

$$
\sum_{j=1}^{m-1} \rho_{j} \phi_{j}\left(\mathbf{p}_{l j}^{T} \mathbf{n}_{1}\right)-\rho_{j} \phi_{j}\left(\mathbf{p}_{l j}^{T} \mathbf{n}_{2}\right)=0 . \quad l=1,2,3
$$

In order that equation 4.2 hold for all $l$, for every $l$ there must be one term in the equation that differs in sign from the other terms ${ }^{1}$.

Now,

$$
\begin{aligned}
& \operatorname{sign}\left[\rho_{j} \phi_{j}\left(\mathbf{p}_{l j}^{T} \mathbf{n}_{1}\right)-\rho_{j} \phi_{j}\left(\mathbf{p}_{l j}^{T} \mathbf{n}_{2}\right)\right] \\
& =\operatorname{sign}\left[\begin{array}{lll}
\mathbf{p}_{l j}^{T} \mathbf{n}_{1}-\mathbf{p}_{l j}^{T} \mathbf{n}_{2} & \cdots & \phi_{j} \text { is monotonically strictly increasing, } \rho_{j}>0
\end{array}\right. \\
& =\operatorname{sign}\left[\mathbf{p}_{l j}^{T} \mathbf{n}\right] \text {. }
\end{aligned}
$$

where, $\mathbf{n}=\mathbf{n}_{1}-\mathbf{n}_{2} \neq 0$.

This leads us to the following lemma.

\footnotetext{
${ }^{1}$ We have to deal with the technicality of assigning a sign to zero. We deal with this as follows: Note that we are interested in cases where $m \geq 3$ in equation 4.1 . Hence, for every $l$, we have at least two terms for equation 4.2. If there are more than two non-zero terms in equation 4.2 , then the zero terms are assigned any sign that we wish. If there is only one non-zero term, then the zero terms are assigned the sign opposite to this term. If all the terms are zero, then we assign differing sign two any two of the zeros and the rest are assigned in any manner whatsoever.
} 
Lemma 4.1 If the matrices $H_{j}, j=1, \cdots, m-1$ are such that $\forall \mathbf{n}$, $\mathbf{n} \neq 0$, there is at least one row of $H_{1} \mathbf{n}, H_{2} \mathbf{n}, \cdots, H_{m-1} \mathbf{n}$ with all entries having the same sign, then equation 4.1 has a globally unique solution for any set of monotonic, strictly increasing functions $\phi_{j}()$.

Proof Let the condition of the lemma be true. Then, given an $\mathbf{n}=\mathbf{n}_{1}-\mathbf{n}_{2} \neq 0$, we can find a row $j$ such that the signs of $\mathbf{p}_{l j}^{T} \mathbf{n}$ are the same for all $l$. Since,

$$
\operatorname{sign}\left[\mathbf{p}_{l j}^{T} \mathbf{n}\right]=\operatorname{sign}\left[\rho_{j} \phi_{j}\left(\mathbf{p}_{l j}^{T} \cdot \mathbf{n}_{1}\right)-\rho_{j} \phi_{j}\left(\mathbf{p}_{l j}^{T} \mathbf{n}_{2}\right)\right],
$$

the sum on the left hand side in equation 4.2 will not yield the right hand side and we will have a unique solution to equation 4.1 .

\subsection{Inversion of three-lobed maps}

For the time being, let us consider only three-lobed maps. We have,

$$
H_{1}=\left(\begin{array}{c}
\mathbf{p}_{11}^{T} \\
\mathbf{p}_{21}^{T} \\
\mathbf{p}_{31}^{T}
\end{array}\right) \quad H_{2}=\left(\begin{array}{c}
\mathbf{p}_{12}^{T} \\
\mathbf{p}_{22}^{T} \\
\mathbf{p}_{32}^{T}
\end{array}\right) .
$$

We will now investigate the manner in which $H_{1}$ and $H_{2}$ affect the sign of elements of the rows of $H_{1} \mathbf{n}$ and $H_{2} \mathbf{n}$. This we do as follows:

- We first isolate those sets of $E^{3}$, such that if $\mathbf{n}$ belongs to them, one row of $H_{1} \mathbf{n}$ and $H_{2} \mathrm{n}$ differs in sign. There are six such sets :

$c_{1}=\left\{\mathbf{n}:\right.$ sign of the 1 st row of $H_{1} \mathbf{n}$ is +ve, sign of the 1 st row of $H_{2} \mathbf{n}$ is -ve $\}-$ $\{0\}$,

$$
=\left\{\mathbf{n}: \mathbf{p}_{11}^{T} \mathbf{n} \geq 0, \mathbf{p}_{12}^{T} \mathbf{n} \leq 0\right\}-\{0\} .
$$

$c_{1}^{*}=\left\{\mathbf{n}:\right.$ sign of the 1 st row of $H_{1} \mathbf{n}$ is -ve, sign of the 1 st row of $H_{2} \mathbf{n}$ is $\left.+v e\right\}-$ $\{0\}$,

$$
=\left\{\mathbf{n}: \mathbf{p}_{11}^{T} \mathbf{n}<0, \mathbf{p}_{12}^{T} \mathbf{n}>0\right\} .
$$

$c_{2}=\left\{\mathbf{n}: \operatorname{sign}\right.$ of the 2 nd row of $H_{1} \mathbf{n}$ is +ve, sign of the 2 nd row of $H_{2} \mathbf{n}$ is -ve $\}-$ $\{0\}$.

$$
=\left\{\mathbf{n}: \mathbf{p}_{21}^{T} \mathbf{n} \geq 0, \mathbf{p}_{22}^{T} \mathbf{n} \leq 0\right\}-\{0\} .
$$


$c_{2}^{*}=\left\{\mathbf{n}:\right.$ sign of the 2 nd row of $H_{1} \mathbf{n}$ is $-\mathrm{ve}$, sign of the 2 nd row of $H_{2} \mathbf{n}$ is $\left.+\mathrm{ve}\right\}-$ $\{0\}$.

$$
=\left\{\mathbf{n}: \mathbf{p}_{21}^{T} \mathbf{n}<0, \mathbf{p}_{22}^{T} \mathrm{n}>0\right\} .
$$

$c_{3}=\left\{\mathbf{n}:\right.$ sign of the 3 rd row of $H_{1} \mathrm{n}$ is $+\mathrm{ve}$, sign of the 3 rd row of $H_{2} \mathrm{n}$ is -ve $\}-$ $\{0\}$.

$$
=\left\{\mathbf{n}: \mathbf{p}_{31}^{T} \mathbf{n} \geq 0, \mathbf{p}_{32}^{T} \mathrm{n} \leq 0\right\}-\{0\} .
$$

$c_{3}^{*}=\left\{\mathbf{n}\right.$ : sign of the $3 \mathrm{rd}$ row of $H_{1} \mathbf{n}$ is -ve, sign of the $3 \mathrm{rd}$ row of $H_{2} \mathbf{n}$ is $\left.+v e\right\}-$ $\{0\}$.

$$
=\left\{\mathbf{n}: \mathbf{p}_{31}^{T} \mathbf{n}<0, \mathbf{p}_{32}^{T} \mathbf{n}>0\right\} .
$$

We will shortly establish that $c_{1}, c_{2}, c_{3}$ do not intersect $c_{1}^{*}, c_{2}^{*}, c_{3}^{*}$ and that the sets $c_{1}+\{0\}, c_{2}+\{0\}, c_{3}+\{0\}, c_{1}^{*}+\{0\}, c_{2}^{*}+\{0\}, c_{3}^{*}+\{0\}$ are convex cones.

- We can then define sets where two rows of $H_{1} \mathrm{n}$ and $H_{2} \mathrm{n}$ differ in sign.

$$
\begin{array}{ll}
C_{1}=c_{1} \cap c_{2} & C_{1}^{*}=c_{1}^{*} \cap c_{2}^{*}, \\
C_{2}=c_{2} \cap c_{3} & C_{2}^{*}=c_{2}^{*} \cap c_{3}^{*}, \\
C_{3}=c_{3} \cap c_{1} & C_{3}^{*}=c_{3}^{*} \cap c_{1}^{*} .
\end{array}
$$

- We can finally define $\mathcal{C}$ and $\mathcal{C}^{*}$ to be $\mathcal{C}=C_{1} \cap C_{2} \cap C_{3}$ and $\mathcal{C}^{*}=C_{1}^{*} \cap C_{2}^{*} \cap C_{3}^{*}$. Note that if either $\mathcal{C}$ or $\mathcal{C}^{*}$ is not empty and if $\mathbf{n} \epsilon \mathcal{C}$ or $\mathcal{C}^{*}$, then conditions of Lemma 4.1 are violated. If the sets $\mathcal{C}$ and $\mathcal{C}^{*}$ are empty, then it follows from Lemma 4.1 that equation 4.1 has a globally unique solution.

Our task is now reduced to identifying the sets $\mathcal{C}$ and $\mathcal{C}^{*}$, and then checking to see if they are empty. This can be simplified greatly by looking at only some of the sets from which $\mathcal{C}$ and $\mathcal{C}^{*}$ are made. The simplification is based on the the properties of these sets as illustrated by the following lemmas:

Lemma 4.2 When point 0 (the origin of $\mathrm{E}^{3}$ ) is added to the sets $c_{j}, c_{j}^{*}, C_{j}, C_{j}^{*}, \mathcal{C}, \mathcal{C}^{*}$, $j=1,2,3$, the resulting sets are convex cones.

Proof We will prove this for $c_{1}$. The proof for other sets follows identical lines. If $\mathbf{x}_{1}, \mathbf{x}_{2} \in c_{1}+\{0\}$, then $\mathbf{p}_{11}^{T} \mathbf{x}_{1} \geq 0 \quad \mathbf{p}_{12}^{T} \mathbf{x}_{1} \leq 0, \quad \mathbf{p}_{11}^{T} \mathbf{x}_{2} \geq 0 \quad \mathbf{p}_{12}^{T} \mathbf{x}_{2} \leq 0$. From the linearity of the inner product, it follows that if $\mathbf{x}=\alpha \mathbf{x}_{1}+(1-\alpha) \mathbf{x}_{2}, 0 \leq \alpha \leq 1$, then

$$
\begin{array}{r}
\mathbf{p}_{11}^{T} \mathbf{x}=\mathbf{p}_{11}^{T}\left\{\alpha \mathbf{x}_{1}+(1-\alpha) \mathbf{x}_{2}\right\}=\alpha \mathbf{p}_{11}^{T} \mathbf{x}_{1}+(1-\alpha) \mathbf{p}_{11}^{T} \mathbf{x}_{2} \geq 0 \\
\text { and similarly } \mathbf{p}_{12}^{T} \mathbf{x}=\alpha \mathbf{p}_{12}^{T} \mathbf{x}_{1}+(1-\alpha) \mathbf{p}_{12}^{T} \mathbf{x}_{2} \leq 0
\end{array}
$$


Thus, $\mathbf{x} \in c_{1}+\{0\}$, and $c_{1}+\{0\}$ is a convex set.

Further, if $\mathbf{x} \in c_{1}+\{0\}$, then for $\alpha \geq 0$, it is easy to see that $\alpha \mathbf{x} \in c_{1}+\{0\}$. Thus $c_{1}+\{0\}$ is a convex cone with 0 as an apex.

Lemma 4.3 The sets $c_{j}, c_{j}^{*} ; C_{j}, C_{j}^{*} ; \mathcal{C}, \mathcal{C}^{*}$ are complementary in the sense that

$$
\mathbf{x} \in c_{j}^{*} \text { iff }-\mathbf{x} \in c_{j}, \quad \mathbf{x} \in C_{j}^{*} \text { iff }-\mathbf{x} \in C_{j}, \quad \mathbf{x} \in \mathcal{C}^{*} \text { iff }-\mathbf{x} \in \mathcal{C} .
$$

If $\mathbf{x} \neq 0$

$$
\mathrm{x} \in c_{j} \text { iff }-\mathrm{x} \in c_{j}^{*}, \quad \mathrm{x} \in C_{j} \text { iff }-\mathrm{x} \in C_{j}^{*}, \mathrm{x} \in \mathcal{C} \text { iff }-\mathrm{x} \in \mathcal{C}^{*} .
$$

Also, it follows that, $c_{j} \cap c_{j}^{*}=\emptyset \quad C_{j} \cap C_{j}^{*}=\emptyset \mathcal{C} \cap \mathcal{C}^{*}=\emptyset$.

Proof The proof follows from the definitions of the sets and the linearity of the inner product.

Lemma 4.3 has an interesting consequence. If $\mathcal{C}$ is empty, then so is $\mathcal{C}^{*}$. Thus, it is sufficient to check for the emptyness of $\mathcal{C}$ only. But, $\mathcal{C}$ is defined in terms of the unstarred sets; and from this it follows that we need to identify the unstarred sets only.

Let us proceed to identify the sets $C_{j}$.

\section{The sets $C_{j}$}

Figure 4.1 provides some visual aid for subsequent theory. We begin with some definitions. With each vector $\mathbf{p}_{l j}$ let us associate a vector $\mathbf{r}_{l j}$ defined by

$$
\mathbf{r}_{l j}=\left(\begin{array}{c}
-\cos \phi \cos \theta \\
-\sin \phi \cos \theta \\
\sin \theta
\end{array}\right) \text {, if } \mathrm{p}_{l j}=\left(\begin{array}{c}
\cos \phi \sin \theta \\
\sin \phi \sin \theta \\
\cos \theta
\end{array}\right) \text {. }
$$

We denote the principal planes by $\Pi_{l}$. Clearly, $\Pi_{l}=\operatorname{span}\left(\mathrm{p}_{l 1}, \mathrm{p}_{l 2}\right)$. Let us define $\mathbf{q}_{l}$ such that $\mathbf{q}_{l} \neq 0$ and $\mathbf{q}_{l} \perp \Pi_{l}$. Since $\Pi_{l}$ contains the z-axis, $\mathbf{q}_{l}$ must lie in the $\mathrm{x}-\mathrm{y}$ plane. If we define planes $\Xi_{l j}=\operatorname{span}\left(\mathbf{r}_{l j}, \mathbf{q}_{l}\right)$, then, we can define vectors $\mathbf{v}_{i}, \mathbf{u}_{i}$ and $\mathbf{w}_{i}$ such that,

$$
\Xi_{11} \cap \Xi_{21}=\left\{a \mathbf{v}_{1}:-\infty \leq a \leq+\infty, \text { z-component of } \mathbf{v}_{1}>0\right\}
$$




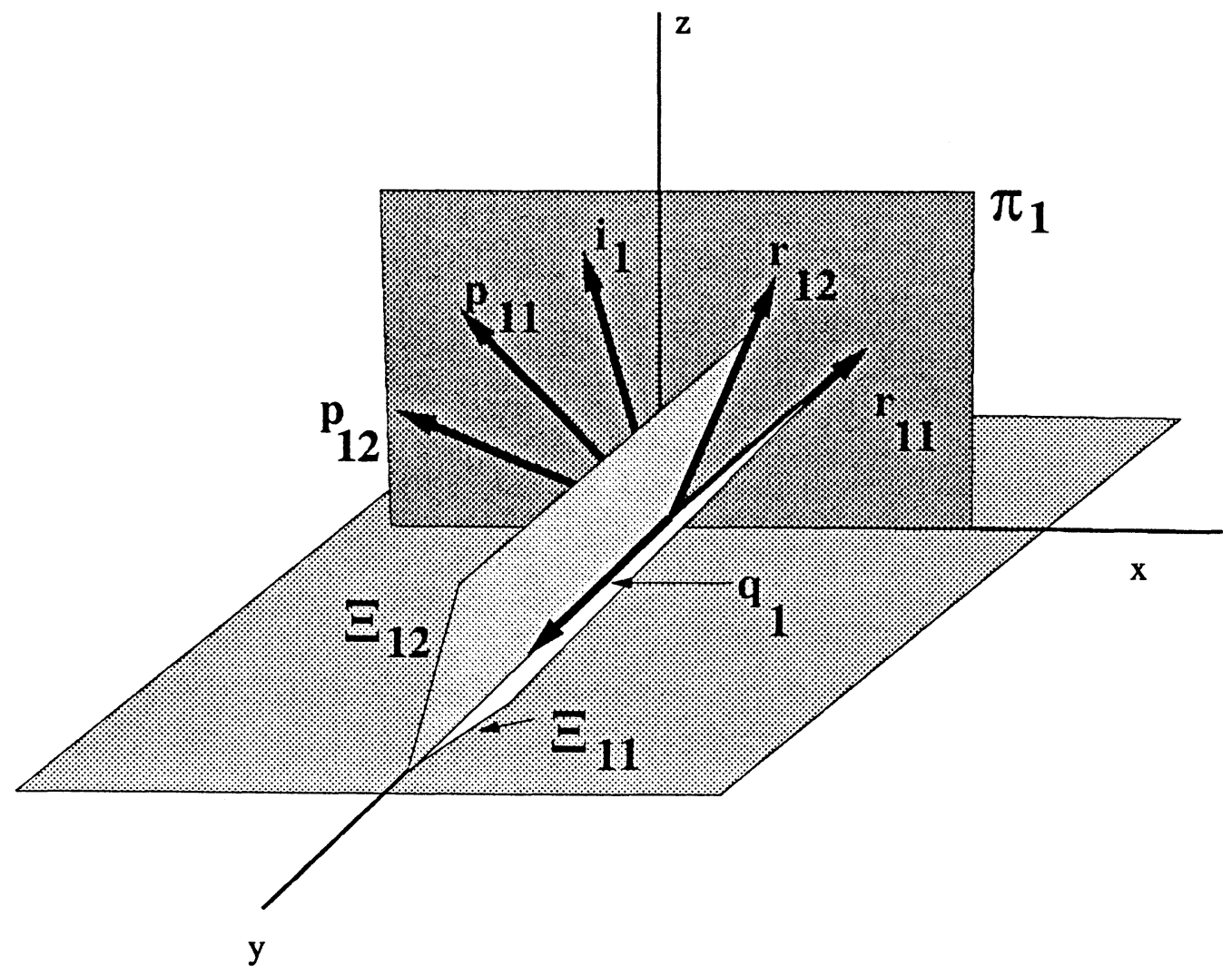

Figure 4.1 Geometry for defining the sets $C_{j}$. 


$$
\begin{aligned}
& \Xi_{11} \cap \Xi_{22}=\left\{a \mathbf{v}_{2}:-\infty \leq a \leq+\infty, \text { z-component of } \mathbf{v}_{2}>0\right\} \\
& \Xi_{12} \cap \Xi_{22}=\left\{a \mathbf{v}_{3}:-\infty \leq a \leq+\infty, \text { z-component of } \mathbf{v}_{3}>0\right\} \\
& \Xi_{12} \cap \Xi_{21}=\left\{a \mathbf{v}_{4}:-\infty \leq a \leq+\infty, \text { z-component of } \mathbf{v}_{4}>0\right\} \\
& \Xi_{31} \cap \Xi_{21}=\left\{a \mathbf{u}_{1}:-\infty \leq a \leq+\infty, \text { z-component of } \mathbf{u}_{1}>0\right\} \\
& \Xi_{31} \cap \Xi_{22}=\left\{a \mathbf{u}_{2}:-\infty \leq a \leq+\infty, \text { z-component of } \mathbf{u}_{2}>0\right\} \\
& \Xi_{32} \cap \Xi_{22}=\left\{a \mathbf{u}_{3}:-\infty \leq a \leq+\infty, \text { z-component of } \mathbf{u}_{3}>0\right\} \\
& \Xi_{32} \cap \Xi_{21}=\left\{a \mathbf{u}_{4}:-\infty \leq a \leq+\infty, \text { z-component of } \mathbf{u}_{4}>0\right\} \\
& \Xi_{11} \cap \Xi_{31}=\left\{a \mathbf{w}_{1}:-\infty \leq a \leq+\infty, \text { z-component of } \mathbf{w}_{1}>0\right\} \\
& \Xi_{11} \cap \Xi_{32}=\left\{a \mathbf{w}_{2}:-\infty \leq a \leq+\infty, \text { z-component of } \mathbf{w}_{2}>0\right\} \\
& \Xi_{12} \cap \Xi_{32}=\left\{a \mathbf{w}_{3}:-\infty \leq a \leq+\infty, \text { z-component of } \mathbf{w}_{3}>0\right\} \\
& \Xi_{12} \cap \Xi_{31}=\left\{a \mathbf{w}_{4}:-\infty \leq a \leq+\infty, \text { z-component of } \mathbf{w}_{4}>0\right\}
\end{aligned}
$$

We first establish that,

Lemma 4.4 The vectors $\mathbf{v}_{i}, \mathbf{u}_{i}$ and $\mathrm{w}_{i}$ as defined above exist and $\mathbf{v}_{i}, \mathbf{u}_{i}, \mathbf{w}_{i} \neq 0$. Further,

$$
\begin{aligned}
& \Xi_{11}=\operatorname{span}\left(\mathbf{v}_{1}, \mathbf{v}_{2}\right)=\operatorname{span}\left(\mathbf{w}_{1}, \mathbf{w}_{3}\right), \quad \Xi_{12}=\operatorname{span}\left(\mathbf{v}_{4}, \mathbf{v}_{3}\right)=\operatorname{span}\left(\mathbf{w}_{3}, \mathbf{w}_{4}\right) \\
& \Xi_{21}=\operatorname{span}\left(\mathbf{v}_{1}, \mathbf{v}_{4}\right)=\operatorname{span}\left(\mathbf{u}_{1}, \mathbf{u}_{4}\right), \quad \Xi_{22}=\operatorname{span}\left(\mathbf{v}_{2}, \mathbf{v}_{3}\right)=\operatorname{span}\left(\mathbf{u}_{2}, \mathbf{u}_{3}\right), \\
& \Xi_{31}=\operatorname{span}\left(\mathbf{u}_{1}, \mathbf{u}_{2}\right)=\operatorname{span}\left(\mathbf{w}_{1}, \mathbf{w}_{4}\right), \quad \Xi_{32}=\operatorname{span}\left(\mathbf{u}_{3}, \mathbf{u}_{4}\right)=\operatorname{span}\left(\mathbf{w}_{2}, \mathbf{w}_{3}\right) .
\end{aligned}
$$

Proof Consider existence first. We will prove this for $\mathbf{v}_{\mathbf{1}}$. The proof for other vectors follows identical lines. Consider the set $S=\Xi_{11} \cap \Xi_{21}$. The set $S$ has dimension 1. Since, $\Xi_{11}=\operatorname{span}\left(\mathbf{r}_{11}, \mathbf{q}_{1}\right)$ and $\Xi_{21}=\operatorname{span}\left(\mathbf{r}_{21}, \mathbf{q}_{2}\right)$, if $\mathbf{x} \in S$, then $\mathbf{p}_{11}^{T} \mathbf{x}=0$ and $\mathbf{p}_{21}^{T} \mathbf{x}=0$. The set $S$, therefore, consists of vectors orthogonal to $\mathbf{p}_{11}$ and $\mathbf{p}_{21}$. As we know that the vector $\mathbf{p}_{11} * \mathbf{p}_{21}$ satisfies this requirement, let us consider $\mathbf{p}_{11} * \mathbf{p}_{21}$ as a likely candidate for $\mathbf{v}_{1}$. The z-component of this vector is $\sin \theta_{11} \sin \theta_{21} \sin \left(\phi_{i_{2}}-\phi_{i_{1}}\right) \neq 0$. Hence, the set $S$ can be written as $a \mathbf{v}_{1}$, where $\mathbf{v}_{1}= \pm \mathbf{p}_{11} * \mathbf{p}_{21}$, the sign being chosen to keep the z-component of $\mathbf{v}_{1}$ strictly greater than zero. This establishes that $\mathbf{v}_{1}$ exists.

We will now show that $\Xi_{11}=\operatorname{span}\left(\mathbf{v}_{1}, \mathbf{v}_{2}\right)$. Note that by definition $\mathbf{v}_{1}, \mathbf{v}_{2} \in \Xi_{11}$. Hence, the result follows if we can show that $\mathbf{v}_{1}$ and $\mathbf{v}_{2}$ are linearly independent. To 
do this, note that $\mathbf{v}_{1} \in \Xi_{21}$ and $\mathbf{v}_{2} \in \Xi_{22}$, so we have a representation for them of the form

$$
\mathbf{v}_{1}=\gamma_{1} \mathbf{q}_{2}+\alpha_{1} \mathbf{r}_{21}, \quad \mathbf{v}_{2}=\gamma_{2} \mathbf{q}_{2}+\alpha_{2} \mathbf{r}_{22}
$$

Since, $\mathbf{r}_{21}$ and $\mathbf{r}_{22}$ are linearly independent and orthogonal to $\mathbf{q}_{2}$, the only way $\mathbf{v}_{1}$ and $\mathbf{v}_{2}$ can be linearly depandant is if $\alpha_{1}=\alpha_{2}=0$. But since the $\mathrm{z}$-component of $\mathbf{q}_{2}$ is zero, that makes the z-component of $\mathbf{v}_{1}$ and $\mathbf{v}_{2}$ zero. This is a contradiction with the defintions of the two vectors. Hence, $\mathbf{v}_{1}$ and $\mathbf{v}_{2}$ are linearly independent and $\Xi_{11}=\operatorname{span}\left(\mathbf{v}_{1}, \mathbf{v}_{2}\right)$.

From the proof of the above lemma we have that

$$
\begin{array}{r}
\mathbf{v}_{1}= \pm \mathbf{p}_{11} * \mathbf{p}_{12}, \mathbf{v}_{2}= \pm \mathrm{p}_{11} * \mathrm{p}_{22}, \mathbf{v}_{3}= \pm \mathrm{p}_{21} * \mathrm{p}_{22}, \mathbf{v}_{4}= \pm \mathrm{p}_{21} * \mathbf{p}_{12} \\
\mathbf{w}_{1}= \pm \mathbf{p}_{11} * \mathbf{p}_{13}, \mathbf{w}_{2}= \pm \mathbf{p}_{11} * \mathbf{p}_{23}, \mathbf{w}_{3}= \pm \mathrm{p}_{21} * \mathbf{p}_{23}, \mathbf{w}_{4}= \pm \mathbf{p}_{21} * \mathbf{p}_{13}
\end{array}
$$

We now come to

Lemma 4.5 The sets $C_{1}, C_{2}, C_{3}$ are given by

$$
\begin{aligned}
& C_{1}=\left\{\mathbf{x}: \mathbf{x}=\alpha \mathbf{v}_{1}+\beta \mathbf{v}_{2}+\gamma \mathbf{v}_{3}+\delta \mathbf{v}_{4} ; \alpha, \beta, \gamma, \delta \geq 0\right\}-\{0\}, \\
& C_{2}=\left\{\mathbf{x}: \mathbf{x}=\alpha \mathbf{u}_{1}+\beta \mathbf{u}_{2}+\gamma \mathbf{u}_{3}+\delta \mathbf{u}_{4} ; \alpha, \beta, \gamma, \delta \geq 0\right\}-\{0\}, \\
& C_{3}=\left\{\mathbf{x}: \mathbf{x}=\alpha \mathbf{w}_{1}+\beta \mathbf{w}_{2}+\gamma \mathbf{w}_{3}+\delta \mathbf{w}_{4} ; \alpha, \beta, \gamma, \delta \geq 0\right\}-\{0\} .
\end{aligned}
$$

Proof We will prove this for $C_{1}$. The proofs for $C_{2}$ and $C_{3}$ follow identical lines. Let $S=\left\{\mathbf{x}: \mathbf{x}=\alpha \mathbf{v}_{1}+\beta \mathbf{v}_{\mathbf{2}}+\gamma \mathbf{v}_{\mathbf{3}}+\delta \mathbf{v}_{\mathbf{4}} ; \alpha, \beta, \gamma, \delta \geq 0\right\}$. If $\mathbf{x} \in S$ and $\mathbf{x} \neq 0$

$$
\begin{array}{rlrl}
\mathbf{p}_{11}^{T} \mathbf{x} & =\alpha \mathbf{p}_{11}^{T} \mathbf{v}_{1}+\beta \mathbf{p}_{11}^{T} \mathbf{v}_{2}+\gamma \mathbf{p}_{11}^{T} \mathbf{v}_{3}+\delta \mathbf{p}_{11}^{T} \mathbf{v}_{\mathbf{4}} \\
& =\beta \mathbf{p}_{11}^{T} \mathbf{v}_{3}+\gamma \mathbf{p}_{11}^{T} \mathbf{v}_{\mathbf{4}} . & \ldots \text { Since } \mathbf{p}_{11}^{T} \mathbf{v}_{1}=\mathbf{p}_{11}^{T} \mathbf{v}_{2}=0 .
\end{array}
$$

But note that since $\mathbf{v}_{3} \in \Xi_{12}$ and $\mathbf{v}_{4} \in \Xi_{12}$ they can be written as

$$
\mathbf{v}_{3}=a_{1} \mathbf{r}_{12}+b_{1} \mathbf{q}_{1}, \quad \mathbf{v}_{4}=a_{2} \mathbf{r}_{12}+b_{2} \mathbf{q}_{1} \text {. }
$$

which gives us $\mathbf{p}_{11}^{T} \mathbf{v}_{3}>0$ and $\mathbf{p}_{11}^{T} \mathbf{v}_{\mathbf{4}}>0$. Thus, $\mathbf{p}_{11}^{T} \mathbf{x} \geq 0$. Using a similar argument, it is possible to show that

$$
\mathrm{p}_{12}^{T} \mathrm{x} \leq 0, \quad \mathrm{p}_{21}^{T} \mathrm{x} \geq 0, \quad \mathrm{p}_{22}^{T} \mathrm{x} \leq 0 .
$$


From the above inequalities we have $\mathrm{x} \in C_{1}$.

Now consider the converse. We will prove this by a contradiction. Assume that $\exists \mathrm{x} \in C_{1}$ such that $\mathrm{x} \notin S$. Note that by definition $S$ is a convex polyhedron. Since $\mathbf{x} \notin S, \mathbf{x}$ has a projection on $S$. Let $\mathbf{y}$ be the projection. We know from standard convex set theory that $\mathrm{y}$ falls on the boundary of $S$. Consider the tangent plane to $S$ at $\mathbf{y}$. Since $S$ is a polyhedron, this plane must contain one of the faces of $S$. For concreteness, let it be the face that contains $\mathbf{v}_{1}$ and $\mathbf{v}_{2}$. Now $\Xi_{11}=\operatorname{span}\left(\mathbf{v}_{1}, \mathbf{v}_{2}\right)$. Hence, $\Xi_{11}$ is the tangent plane that seperates $S$ from $\mathrm{x}$. Since $\mathrm{p}_{11} \perp \Xi_{11}$, for all $\mathrm{s} \in S$, $\operatorname{sign}\left[\mathbf{p}_{11}^{T} \mathbf{x}\right] \neq \operatorname{sign}\left[\mathbf{p}_{11}^{T} \mathbf{s}\right]$.

Now, from the first half of this proof we know that if $\mathrm{s} \in S$, then $\mathrm{s} \in C_{1}$. Thus $\mathbf{p}_{11}^{T} \mathbf{s}$ has the correct sign a term should if have if $\mathbf{s}$ is to be in $C_{1}$. We also know that since $\mathbf{x} \in C_{1}, \mathbf{p}_{11}^{T} \mathbf{x}$ has the correct sign too. Thus, sign $\left[\mathbf{p}_{11}^{T} \mathbf{x}\right]=\operatorname{sign}\left[\mathbf{p}_{11}^{T} \mathbf{s}\right]$, and we get a contradiction.

\section{The set $\mathcal{C}$}

Finally, we consider $\mathcal{C}$. The test for the emptyness of $\mathcal{C}$ can be simplified by using the following lemma:

Lemma 4.6 If any two sets of $C_{1}, C_{2}$ and $C_{3}$ are disjoint, then $\mathcal{C}=\emptyset$.

Proof The intersection of any two sets from $C_{1}, C_{2}$ and $C_{3}$ decides the signs of all three rows of $H_{1} \mathbf{n}$ and $H_{2} \mathbf{n}$ as can be seen from the definitions of the sets. Thus, if any two of the sets are disjoint, all of them are and the lemma follows as a consequence.

Now, putting everything together, we get

Theorem 4.1 If any two of the sets $C_{1}, C_{2}$ and $C_{3}$ as defined in Lemma 4.5 are disjoint, then the normalized photometric stereo equation has a globally unique solution.

Proof The proof follows from Lemma 4.6 and Lemma 4.1.

To apply this theorem we need a test to check for the disjointedness of any two of $C_{1}, C_{2}$ and $C_{3}$. 
The test for disjointedness of $C_{1}$ and $C_{3}$

From lemma 4.5 it follows that $C_{1}+\{0\}$ and $C_{3}+\{0\}$ are convex polyhedrons. Their disjointedness can be checked by searching for a plane that separates all points of $C_{1}+\{0\}$ and $C_{3}+\{0\}$ except the point 0 . To do this, it is sufficient to check whether the planes spanned by the pairs $\mathrm{w}_{1}, \mathbf{w}_{2}$ and $\mathrm{w}_{2}, \mathbf{w}_{3}$ and $\mathbf{w}_{3}, \mathbf{w}_{4}$ and $\mathbf{w}_{4}, \mathbf{w}_{1}$ separate the vectors $\mathbf{v}_{1}, \mathbf{v}_{2}, \mathbf{v}_{3}$, and $\mathbf{v}_{4}$ from $\mathrm{w}_{1}, \mathrm{w}_{2}, \mathrm{w}_{3}$, and $\mathrm{w}_{4}$. This is the test that shows whether three light sources are sufficient for a globally unique inversion.

\subsection{Three-lobed Isotropic Reflectance maps}

Further analytical mileage can be obtained from the test, by restricting the three lobed map to be isotropic and assuming a symmetrical placement of lights. We will assume that

$$
0<\theta_{11}=\theta_{21}=\theta_{31}=\theta_{1}<\theta_{12}=\theta_{22}=\theta_{32}=\theta_{2}<\pi / 2
$$

and,

$$
\phi_{1}=0,0<\phi_{2}=-\phi_{3}=\phi<\pi .
$$

See figure 4.2 for an illustration.

We now get,

$$
\begin{array}{ll}
\mathbf{v}_{\mathbf{1}}=\left(\begin{array}{c}
-\sin \theta_{1} \cos \theta_{1} \sin \phi \\
-\sin \theta_{1} \cos \theta_{1}(1-\cos \phi) \\
\sin ^{2} \theta_{1} \sin \phi
\end{array}\right), & \mathbf{v}_{2}=\left(\begin{array}{c}
-\sin \theta_{2} \cos \theta_{1} \sin \phi \\
-\sin \theta_{1} \cos \theta_{2}\left(1-\cos \phi \tan \theta_{2} / \tan \theta_{1}\right) \\
\sin \theta_{1} \sin \theta_{2} \sin \phi
\end{array}\right), \\
\mathbf{v}_{3}=\left(\begin{array}{c}
-\sin \theta_{2} \cos \theta_{2} \sin \phi \\
-\sin \theta_{2} \cos \theta_{2}(1-\cos \phi) \\
\sin \theta_{1} \cos \theta_{2} \sin \phi \\
\sin \theta_{2} \sin \phi
\end{array}\right), & \mathbf{v}_{4}=\left(\begin{array}{c}
-\cos \theta_{1} \sin \theta_{2}\left(1-\cos \phi \tan \theta_{1} / \tan \theta_{2}\right) \\
\sin \theta_{1} \sin \theta_{2} \sin \phi
\end{array}\right), \\
\mathbf{w}_{1}=\left(\begin{array}{c}
-\sin \theta_{1} \cos \theta_{1} \sin \phi \\
\sin \theta_{1} \cos \theta_{1}(1-\cos \phi) \\
\sin \theta_{2} \cos \theta_{1} \sin \phi
\end{array}\right), & \mathbf{w}_{2}=\left(\begin{array}{c}
\sin \theta_{1} \cos \theta_{2}\left(1-\cos \phi \tan \theta_{2} / \tan \theta_{1}\right) \\
\sin \theta_{1} \sin \theta_{2} \sin \phi
\end{array}\right), \\
\mathbf{w}_{3}=\left(\begin{array}{c}
-\sin \theta_{1} \cos \theta_{2} \sin \phi \\
\sin \theta_{2} \cos \theta_{2} \sin \phi
\end{array}\right) &
\end{array}
$$



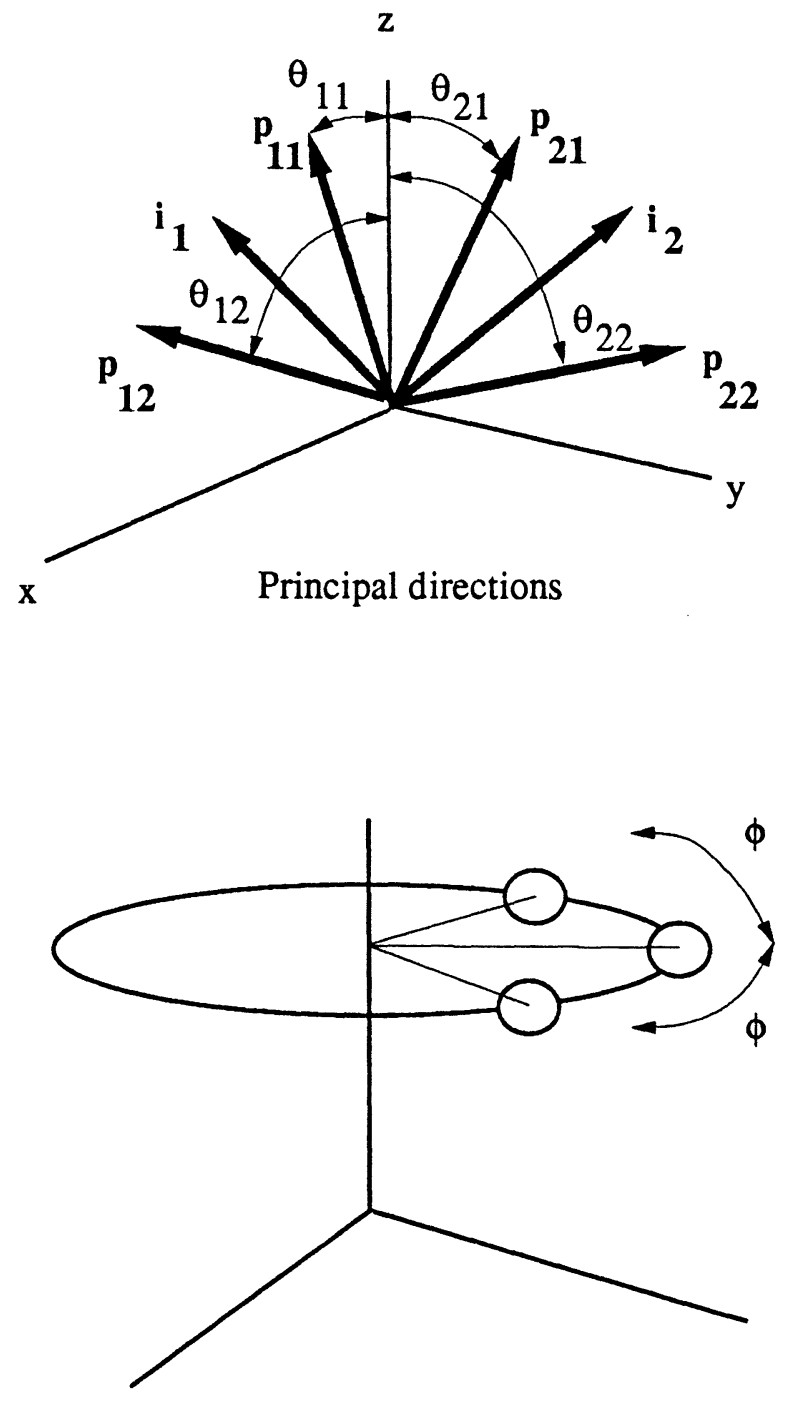

Light source placement

Figure 4.2 Principal Directions and Light Placement for Three Lobed map. 
Note that the signs of the $\mathrm{x}$ and $\mathrm{z}$ components are the same for all $\mathbf{v}$ and $\mathbf{w}$. Since $\theta_{1}<\theta_{2}, \tan \theta_{1}<\tan \theta_{2}$, and the sign of the $y$ component of $\mathbf{v}_{1}, \mathbf{v}_{3}$, and $\mathbf{v}_{4}$ is negative, while the sign of the $y$ component of $\mathrm{w}_{1}, \mathrm{w}_{3}$, and $\mathrm{w}_{4}$ is positive. Clearly, if we make the sign of the y component of $\mathrm{v}_{2}$ negative and that of $\mathrm{w}_{2}$ positive then $C_{1}$ and $C_{3}$ will be disjoint. Hence, we have the condition $\cos \phi<\tan \theta_{1} / \tan \theta_{2}$. This easily translates into

$$
\phi>\cos ^{-1}\left(\frac{\tan \theta_{1}}{\tan \theta_{2}}\right) .
$$

We have proved:

Theorem 4.2 If an isotropic three-lobed reflectance map is used in the normalized photometric stereo equation (4.1), and the lights are placed at a constant zenith angle according to equation (4.3), then the photometric stereo equation has a globally unique solution if the inequality (4.4) holds.

Two observations about the inequality (4.4) are in order:

1. For the class of reflectance maps described by (3.19), we have $\theta_{1}=\theta_{2} / 2$. In general, if we consider $\theta_{1}=\theta_{2} / \beta$, where $\beta>0$, then, we have

$$
1 / \beta=\frac{\theta_{1}}{\theta_{2}}>\frac{\tan \theta_{1}}{\tan \theta_{2}}
$$

Thus, we can set $\phi \geq \cos ^{-1} \frac{1}{\beta}$ and always satisfy the inequality (4.4). For $\beta=2$ we get $\phi \geq 60^{\circ}$. Hence, for the physical reflectance map, three light sources placed at a constant zenith angle and according to equation (4.3) with the azimuth angle between all consecutive light sources greater than or equal to $60^{\circ}$ will cause a globally unique inversion of the normalized photometric stereo equation. Silver [47] used this configuration with the azimuth angle separation of $120^{\circ}$ between any two lights. That is why he was able to reconstruct surfaces using only three light sources.

2. Note that even as $\beta \rightarrow \infty$, if we keep $\phi>90^{\circ}$ the inequality (4.4) will always be satisfied.

\subsection{Inversion of the m-lobed map}

The results for the three-lobed map can be extended very simply to the m-lobed map. Recall that for the m-lobed map we have $m-1$ principal directions $\mathbf{p}_{l j}$ associated with 
every light source direction $i_{l}$. All the principal directions lie in the principal plane $\Pi_{l}$ and their zenith angles $\theta_{l j}$ are strictly increasing with $j$, i.e., $0<\theta_{l j}<\theta_{l j+1}<\pi / 2$. We now have $m-1 H_{j}$ matrices and in order to apply Lemma 3.1 to this case, we have to find a condition where at least one of the rows of $H_{j} \mathbf{n}$ has the same sign in all its entries. Considering the $l^{\text {th }}$ row of $H_{j} \mathrm{n}$ we have,

Lemma 4.7 For any given $\mathbf{n}$, the numbers $\mathbf{p}_{l j}^{T} \mathbf{n}, j=1, \cdots, m-1$ will have the same sign iff

$$
\mathbf{p}_{l 1}^{T} \mathbf{n} \geq 0 \quad \mathrm{p}_{l m-1}^{T} \mathrm{n} \geq 0
$$

or

$$
\begin{array}{lll}
\mathrm{p}_{l 1}^{T} \mathrm{n}<0 & \mathrm{p}_{l m-1}^{T} \mathrm{n}<0 .
\end{array}
$$

Proof Consider the if part of the if and only if. Let $\mathbf{n}=\left(\sin \theta_{n} \cos \phi_{n} \sin \theta_{n} \sin \phi_{n} \cos \theta_{n}\right)^{T}$. Then,

$$
\mathbf{p}_{l k}^{T} \mathbf{n}=\cos \theta_{n} \cos \theta_{l k}-\sin \theta_{n} \sin \theta_{l k} \cos \left(\phi_{n}-\phi_{l}\right) .
$$

From $\mathbf{p}_{l k}^{T} \mathbf{n} \geq 0$ we get $\tan \theta_{l k} \leq 1 / \tan \theta_{n} \cos \left(\phi_{n}-\phi_{l}\right)$. Note that the right hand side of this inequality is independent of $k$. Further, since $\tan \theta_{l k}$ is monotonically increasing function of $\theta_{l k}$ which is a monotonically increasing function of $k$, if the above inequality of true for $\theta_{l 1}$ and $\theta_{l m-1}$, then it must be true for all $\theta_{l k}$ such that $\theta_{l 1}<\theta_{l k}<\theta_{l m-1}$.

The proof for the negative sign is similar.

The only if part of the proof follows from the definition. If all numbers, $\mathbf{p}_{l j}^{T} \mathbf{n}$ are non-negative, then any two of them are, and specifically $\mathbf{p}_{l 1}^{T} \mathbf{n} \geq 0$ and $\mathbf{p}_{l m-1}^{T} \mathbf{n} \geq 0$.

Lemma 4.7 assures us that we can check for the equality of signs of $H_{l} \mathbf{n}$ by checking for equality of signs of the rows of only two vectors $H_{1} \mathbf{n}$ and $H_{m-1} \mathbf{n}$. But this problem is the same as the three-lobed problem where we use $H_{1}$ and $H_{m-1}$ instead of $H_{1}$ and $H_{2}$. This leads us to

Theorem 4.3 The conclusions of Theorems 4.2 and 3.2 are valid for the m-lobed map if $H_{1}$ and $H_{m-1}$ are used in those theorems instead of $H_{1}$ and $\mathrm{H}_{2}$. 
Turning our attention to the isotropic m-lobed map, let us assume that we have three light sources placed at a constant zenith angle according to equation (4.3). Since the map is isotropic, we know that $\theta_{l 1}$ and $\theta_{l m-1}$ are independent of $l$, and in fact $\theta_{l 1} / \theta_{l m-1}=1 / \beta$ where, $\beta$ is a positive real number. This condition is the same as the one in the remark following the inequality (4.4) and hence we know that the normalized photometric stereo equation is globally uniquely invertible if the three light sources are placed with $\phi>90^{\circ}$.

\subsection{Experimental Results.}

We have managed to establish that under some rather mild conditions on light source placements, it is possible to get a globally unique solution to the normalized photometric stereo equation with three light sources. In this section, we will experimentally validate this result. Recall from chapter 2 that the benchmark exercise for photometric stereo is reconstruction of a spherical surface. We will reconstruct two spherical surfaces. The first spherical surface is Lambertian and we will reconstruct it using a Lambertian reflectance map. The other surface is non-Lambertian and we will reconstruct it in two ways. First, we will use un-normalized Lambertian photometric stereo which is the classical technique as suggested in [42] [15] [39] and then with the normalized photometric stereo equation and the physical reflectance map of chapter 3. We will not only establish that three light sources are sufficient to get globally unique reconstructions with the normalized photometric stereo, but will also establish that using the physical reflectance map decreases the error in the reconstruction to an order of magnitude lower than the classical technique. To be perfectly fair, we will use the same three images while using the un-normalized Lambertian photometric stereo and the normalized non-Lambertian photometric stereo.

If a photometric stereo technique reconstructs a sphere with good fidelty, we expect it to reconstruct other surfaces with high fidelity too. To check this, we will use the normalized photometric stereo equation with the physical reflectance map to reconstruct two other surfaces.

\section{Reconstruction of Spherical Surfaces.}

Figure 4.3 shows the two spherical surfaces. The surface on the right is the same as that of Fig. 3.14. We know from chapter 3 that this surface is non-Lambertian and 


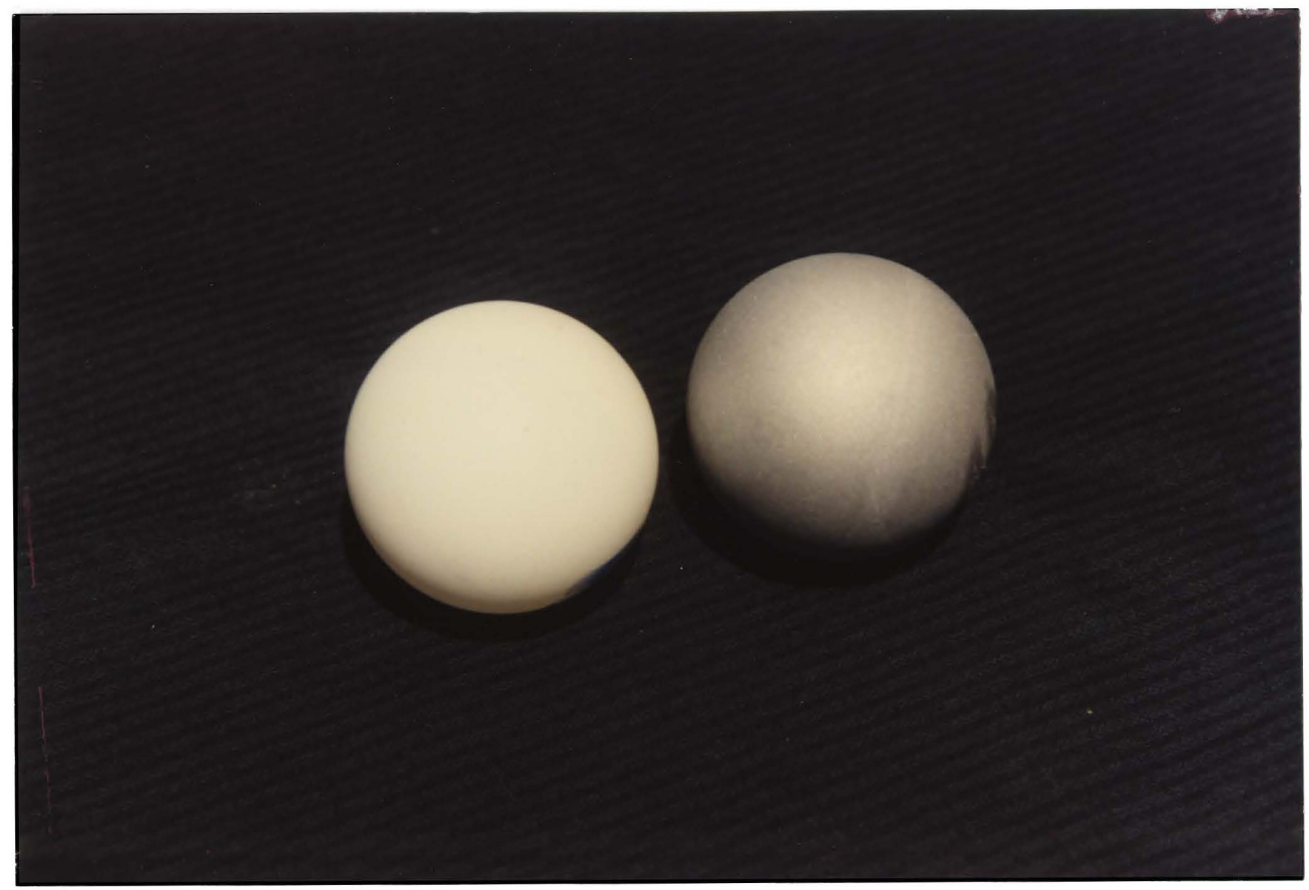

Figure 4.3 Two spherical surfaces.

that its reflectance map is given by equation 3.19 with

$$
\rho_{f s c}=1.00 \quad \rho_{\text {norm }}=0.5 \quad \rho_{b s c}=0.0 \quad c=2.578 .
$$

Figure 4.4 shows the intensity recorded along the principal plane in the image when the surface on the left was illuminated by a light source from a zenith angle of $25^{\circ}$. Figure 4.4 also shows a fit of the Lambertian model to the data. From the closeness of the fit it is clear that this surface is Lambertian.

Next, images of both surfaces were obtained by using three lights placed symmetrically at azimuth angles of $0^{\circ}, 120^{\circ}$ and $-120^{\circ}$ and at a zenith angle of $25^{\circ}$. Figure 4.5 shows the reconstruction of the non-Lambertian surface using a Lambertian model, while figure 4.6 shows the reconstruction using the same three images and the above non-Lambertian model. The reconstruction was obtained from the experimental data by using a standard Levenberg-Marquardt algorithm [30] for solving the non-linear normalized photometric stereo equation (4.1). Note that the Lambertian model yields a distinctly peaky and non-spherical surface. Figure 4.7 shows the depth profiles of the reconstructions in the $x-z$ plane. The distortion due to forcing a Lambertian model on a non-Lambertian surface is clearly evident. 


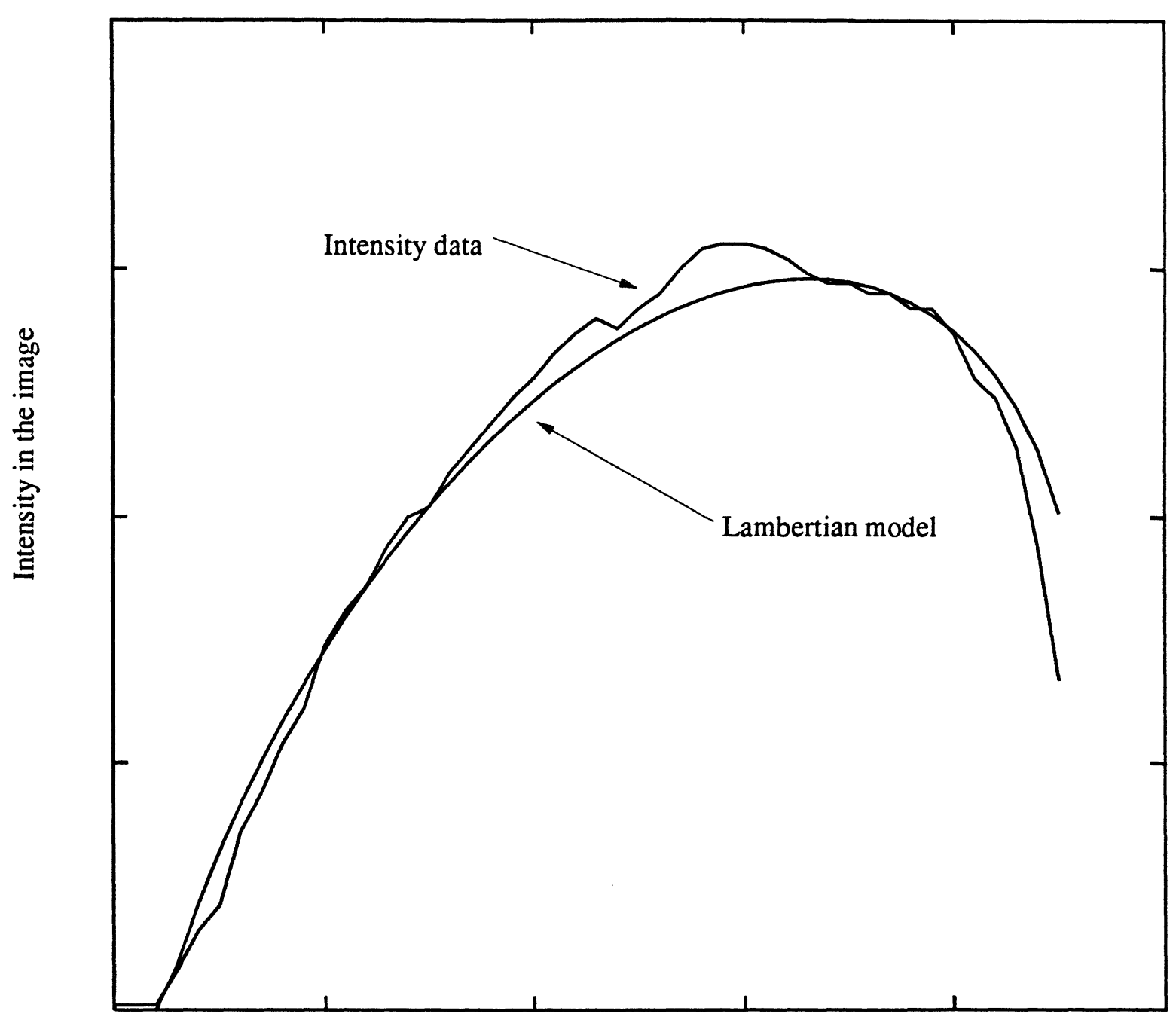

Distance along the principle plane

Figure 4.4 Intensity in the principal plane for the Lambertian sphere. 


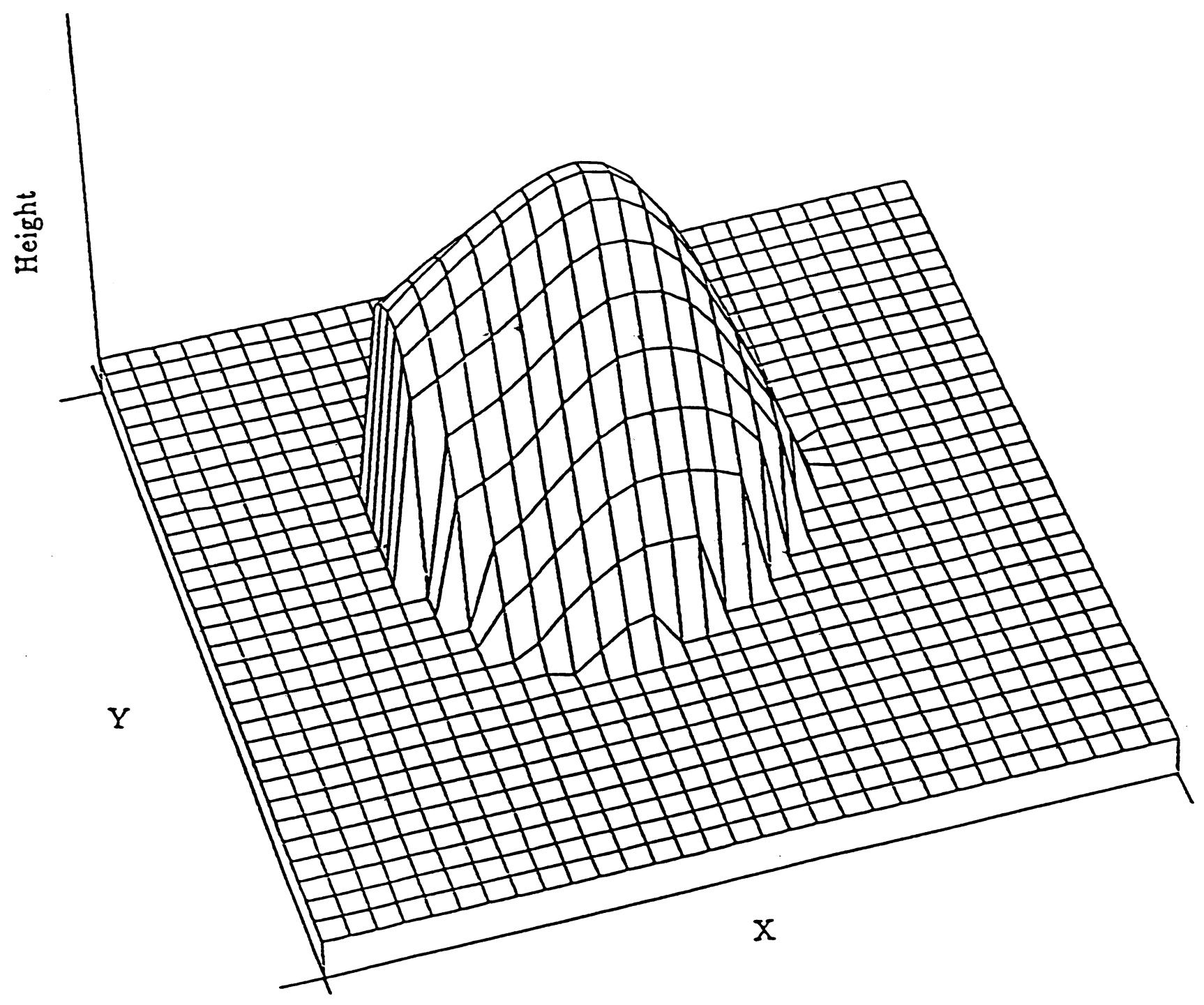

Figure 4.5 Reconstruction of the non-Lambertian surface using the Lambertian model. 


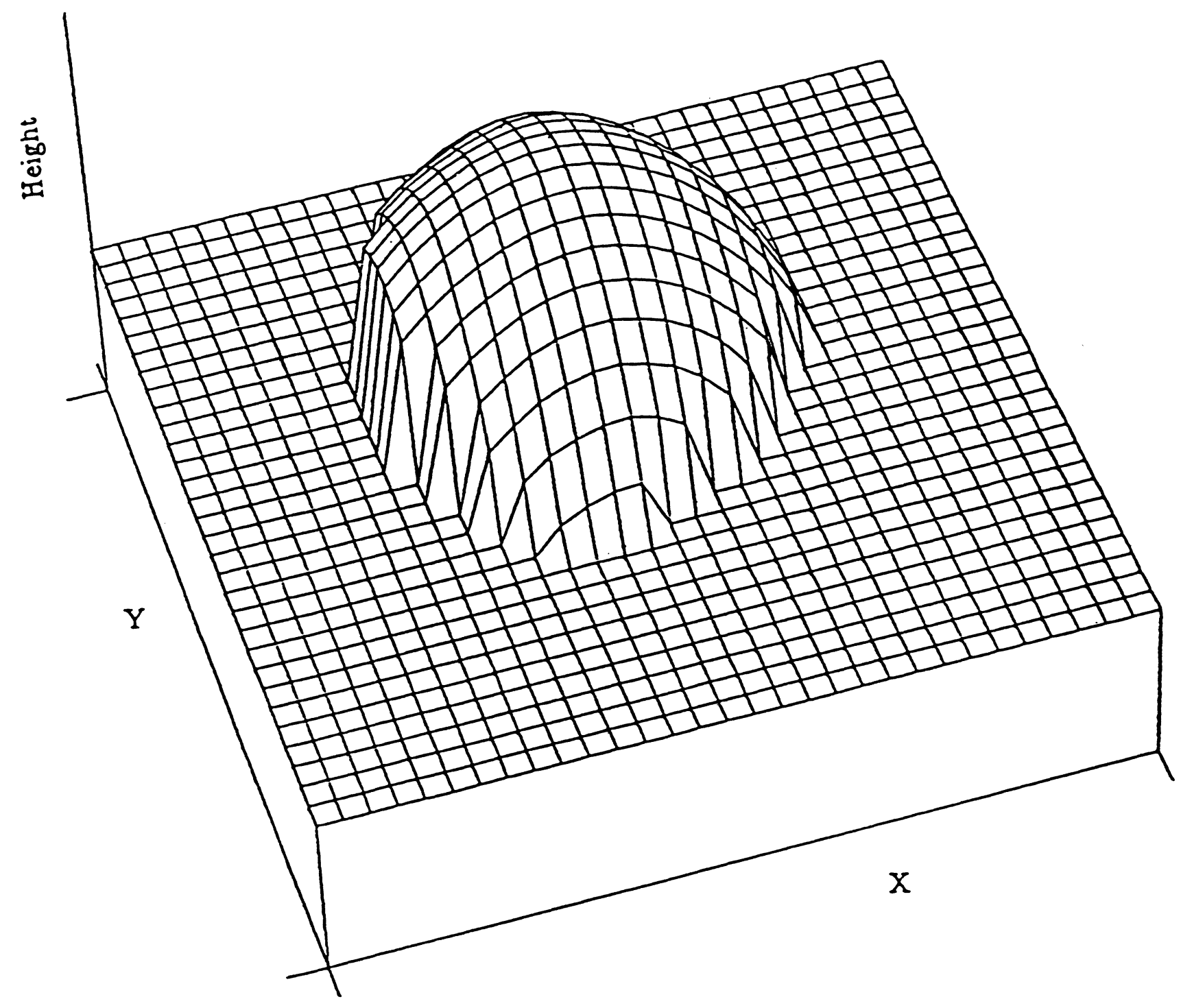

Figure 4.6 Reconstruction of the non-Lambertian surface using the non-Lambertian model. 


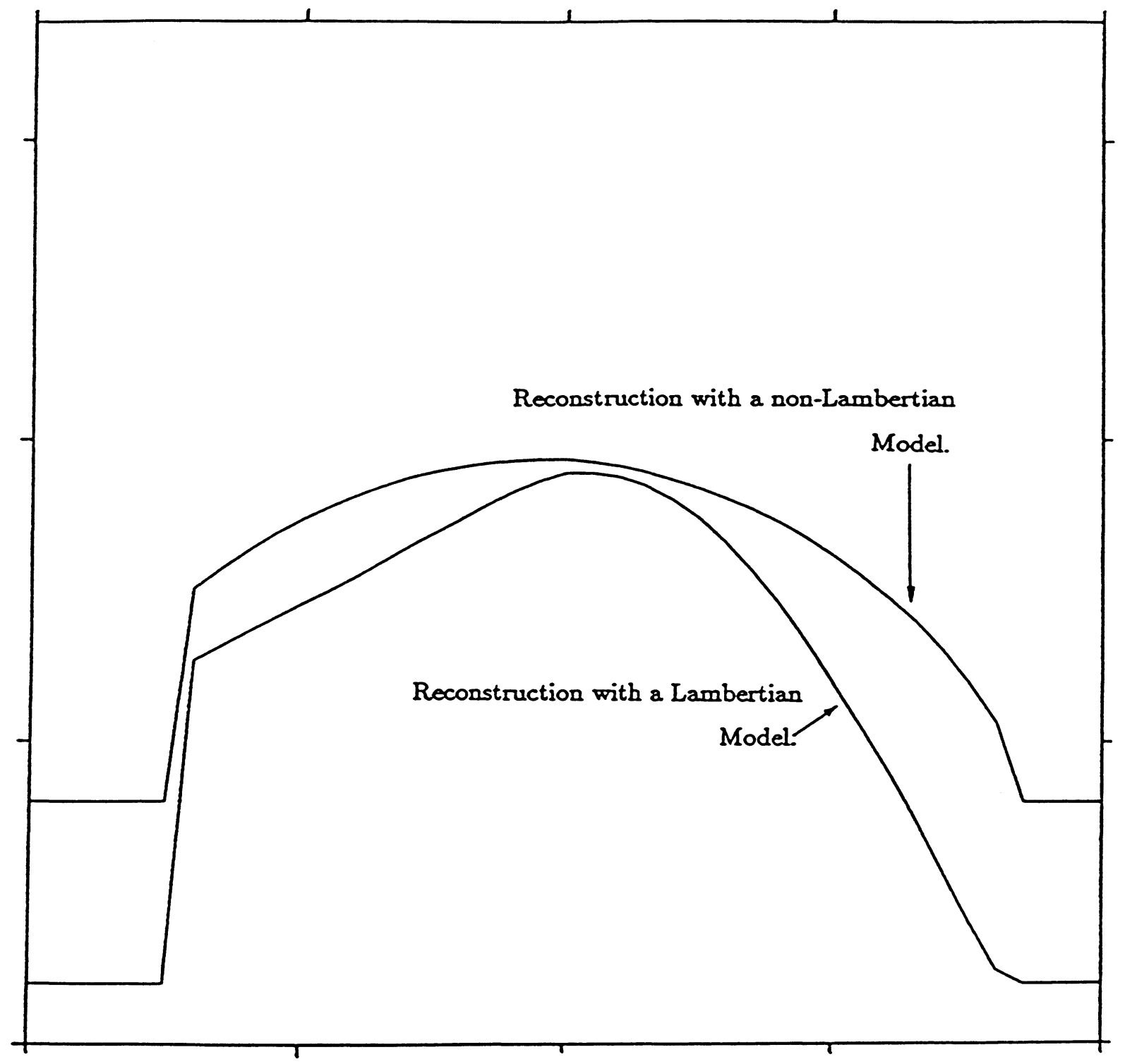

Figure 4.7 Depth profiles of reconstruction. 


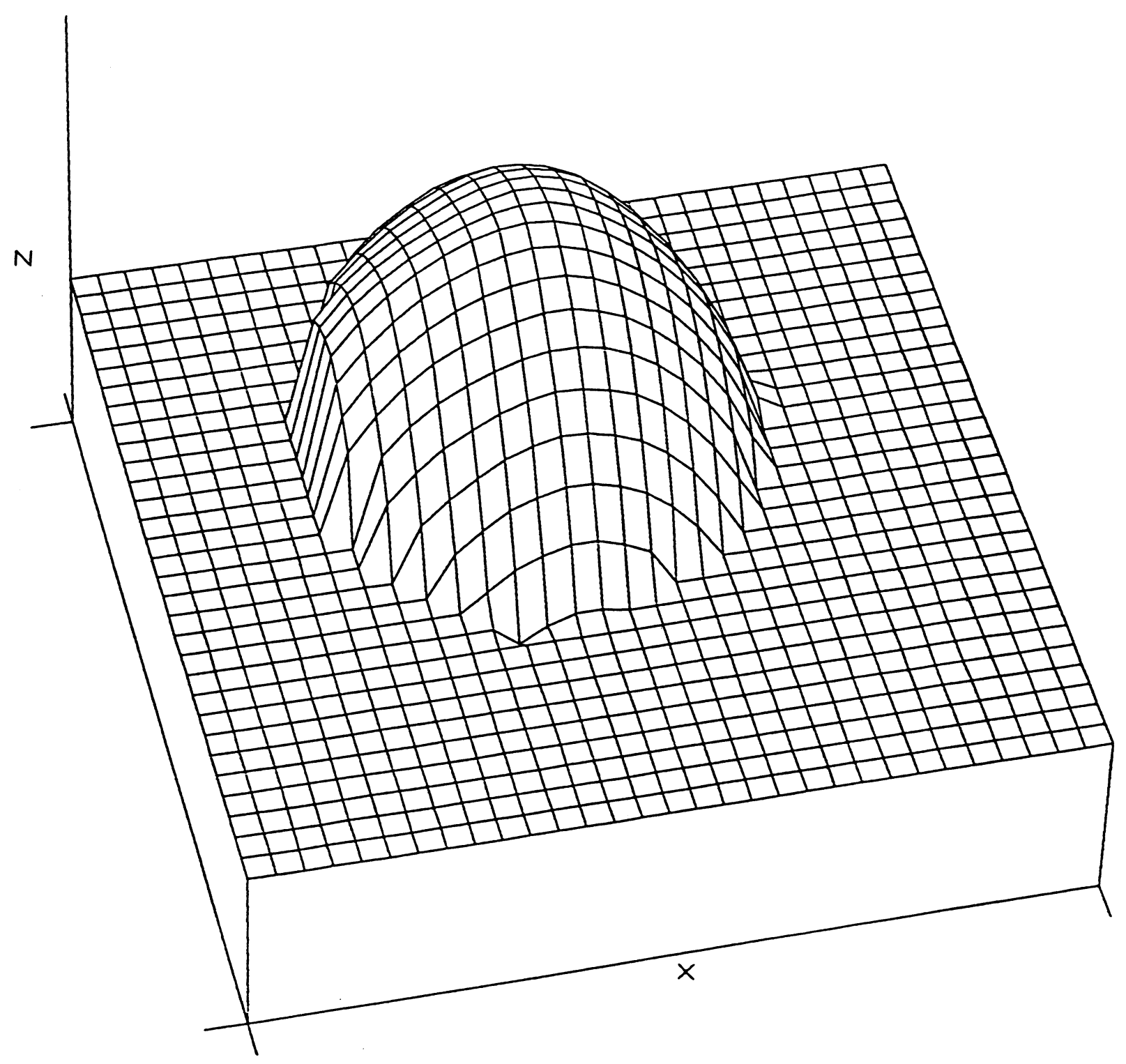

Figure 4.8 Reconstruction of a Lambertian surface using a Lambertian model. 
We present figure 4.8 for comparison with figure 4.6. Figure 4.8 shows the reconstruction of the Lambertian surface of figure 4.3 using a Lambertian model. A comparison of figure 4.6, 4.8 and figure 4.5 shows distinctly that unless the correct reflectance map is used, the reconstructions can be quite poor.

An error analysis of this data was conducted as follows. A spherical surface was fit to each of the reconstructions and the r.m.s. error between the best fit sphere surface normals and the reconstructed surface normals was computed. The various formulae used in computing this are given below.

\section{The Best Fit Sphere}

After photometric stereo was used on each sphere, the results were stored as two images - a $\phi$ image and a $\theta$ image. The $\phi$ image stored the azimuth angles of the reconstructed surface normals and the $\theta$ image stored the zenith angles of the normal.

A third image was also created. This image, called the "Domain Image" contained information about the spatial extent of the region that was reconstructed, i.e., the image had 1's in those pixels where the spherical surface was visible and 0's in places where it wasn't. This was done by simply thresholding the three images used for reconstruction and "anding" the resulting images.

Let us denote the set of pixels in the "Domain Image" which have a value of 1 by $\Omega$. Also, let $\phi(i, j)$ and $\theta(i, j)$ denote the azimuth and zenith angles of the reconstructed normals at the pixel $i, j$. Then, the reconstructed surface normal at the $i, j$ th pixel is

$$
\mathbf{n}(i, j)=\left(\begin{array}{c}
\cos \phi(i, j) \sin \theta(i, j) \\
\sin \phi(i, j) \sin \theta(i, j) \\
\cos \theta(i, j)
\end{array}\right)
$$

If we assume that the sphere had a center at $x_{o}, y_{o}$ and a radius of $r$ then the surface normal at the $i, j$ th pixel should be

$$
\tilde{\mathbf{n}}(i, j)=\left(\begin{array}{c}
\cos \tilde{\phi}(i, j) \sin \tilde{\theta}(i, j) \\
\sin \tilde{\phi}(i, j) \sin \tilde{\theta}(i, j) \\
\cos \tilde{\theta}(i, j)
\end{array}\right)
$$

where, $\tilde{\phi}(i, j)=\arctan \frac{y_{o}-j}{x_{o}-i}$ and $\tilde{\theta}(i, j)=\arcsin \frac{r-\sqrt{\left(x_{o}-i\right)^{2}+\left(y_{o}-j\right)^{2}}}{r}$. The net error in this model is

$$
\Gamma\left(x_{o}, y_{o}, r\right)=\frac{1}{N} \sum_{i, j \in \Omega}(\mathbf{n}(i, j)-\tilde{\mathbf{n}})^{T}(\mathbf{n}(i, j)-\tilde{\mathbf{n}}) .
$$


where, $N$ is the number of elements of $\Omega$.

A gradient descent type algorithm was used to minimize $\Gamma()$ with respect to $x_{o}, y_{o}, r$. The resulting numbers were used as the best fit sphere. The r.m.s error was computed as $\sqrt{\Gamma()}$. This number was converted into an angle value as follows (see figure 4.9)

$$
\Delta \theta_{\text {err }}=2 \frac{\Delta \theta_{\text {err }}}{2} \simeq 2 \sin \frac{\Delta \theta_{\text {err }}}{2} \simeq \sqrt{\Gamma()} .
$$

Table 4.5 shows the results of computing this for the surfaces of figures 4.54 .64 .8

\begin{tabular}{|c|c|c|c|c|}
\hline Type of reconstruction & $x_{o}$ & $y_{\circ}$ & $r$ & $\Delta \theta_{\text {err }}$ \\
\hline Lambertian surface, Lambertian reconstruction & 28.18 & 21.71 & 20.75 & $4.787^{\circ}$ \\
\hline Non-Lambertian surface, Lambertian reconstruction & 24.86 & 28.15 & 21.40 & $19.46^{\circ}$ \\
\hline Non-Lambertian surface, Non-Lambertian reconstruction & 29.21 & 24.49 & 21.86 & $1.82^{\circ}$ \\
\hline
\end{tabular}

Note that for the non-Lambertian surface, the error decreased by an order of magnitude as the reconstruction technique was changed from Lambertian to nonLambertian.

When the right reflectance map is used, the r.m.s. error in the reconstruction is $1.82^{\circ}$ for the non-Lambertian surface and $4.787^{\circ}$ for the Lambertian surface. These numbers are comparable to previously reported values in the literature [42] [39].

\section{Other Reconstructions}

Figure 4.10 shows a bottle and Figure 4.11 an inverted spoon. Figures 4.12 and 4.13

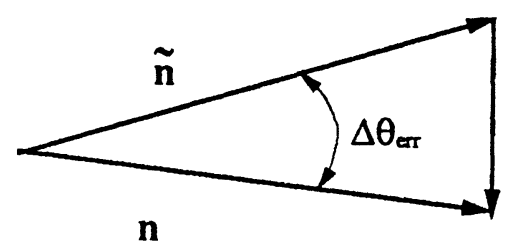

Figure 4.9 Error in the surface normal reconstruction. 


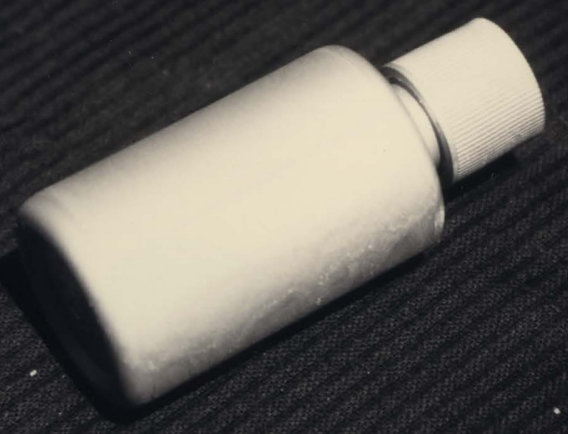

Figure 4.10 Image of a cylinder.

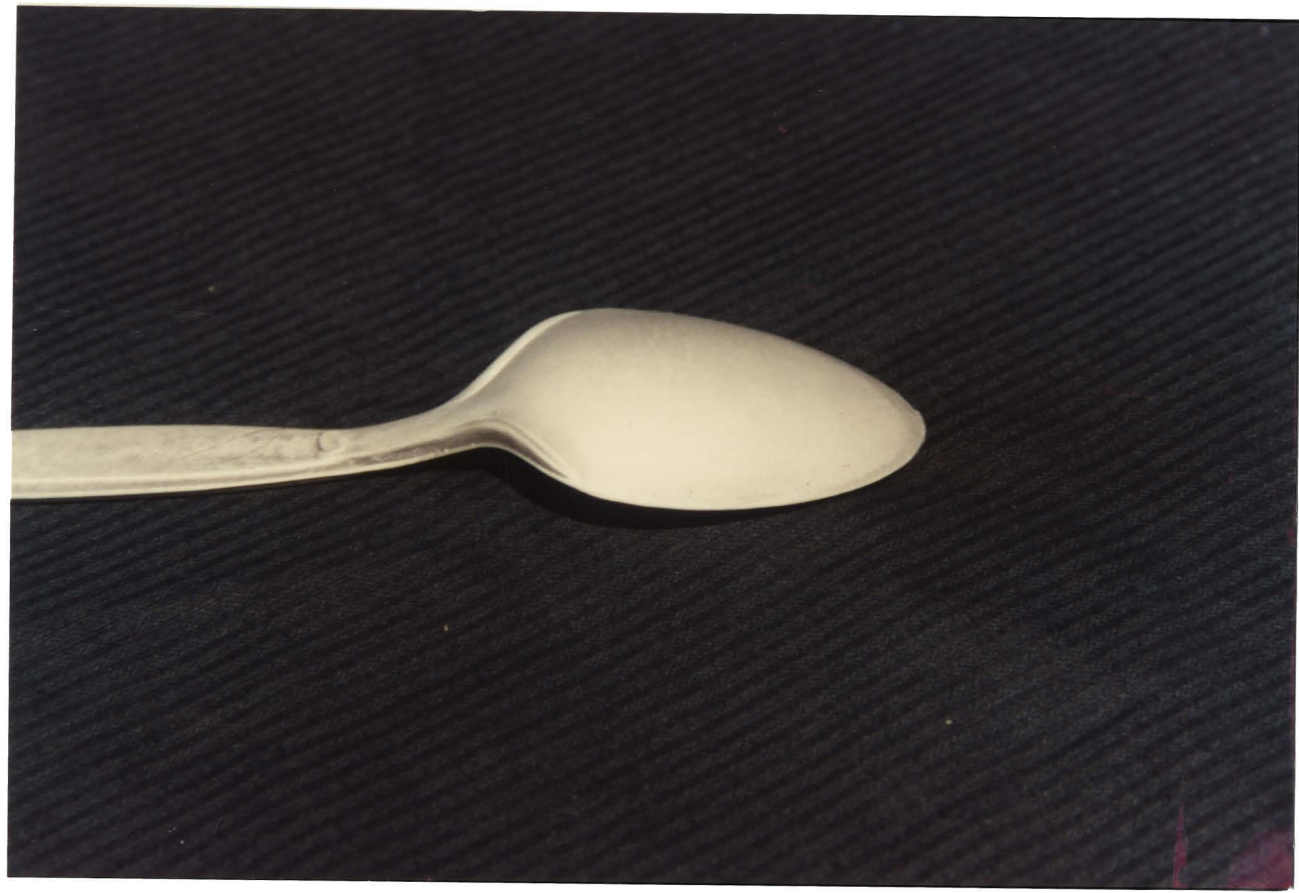

Figure 4.11 Image of a spoon. 


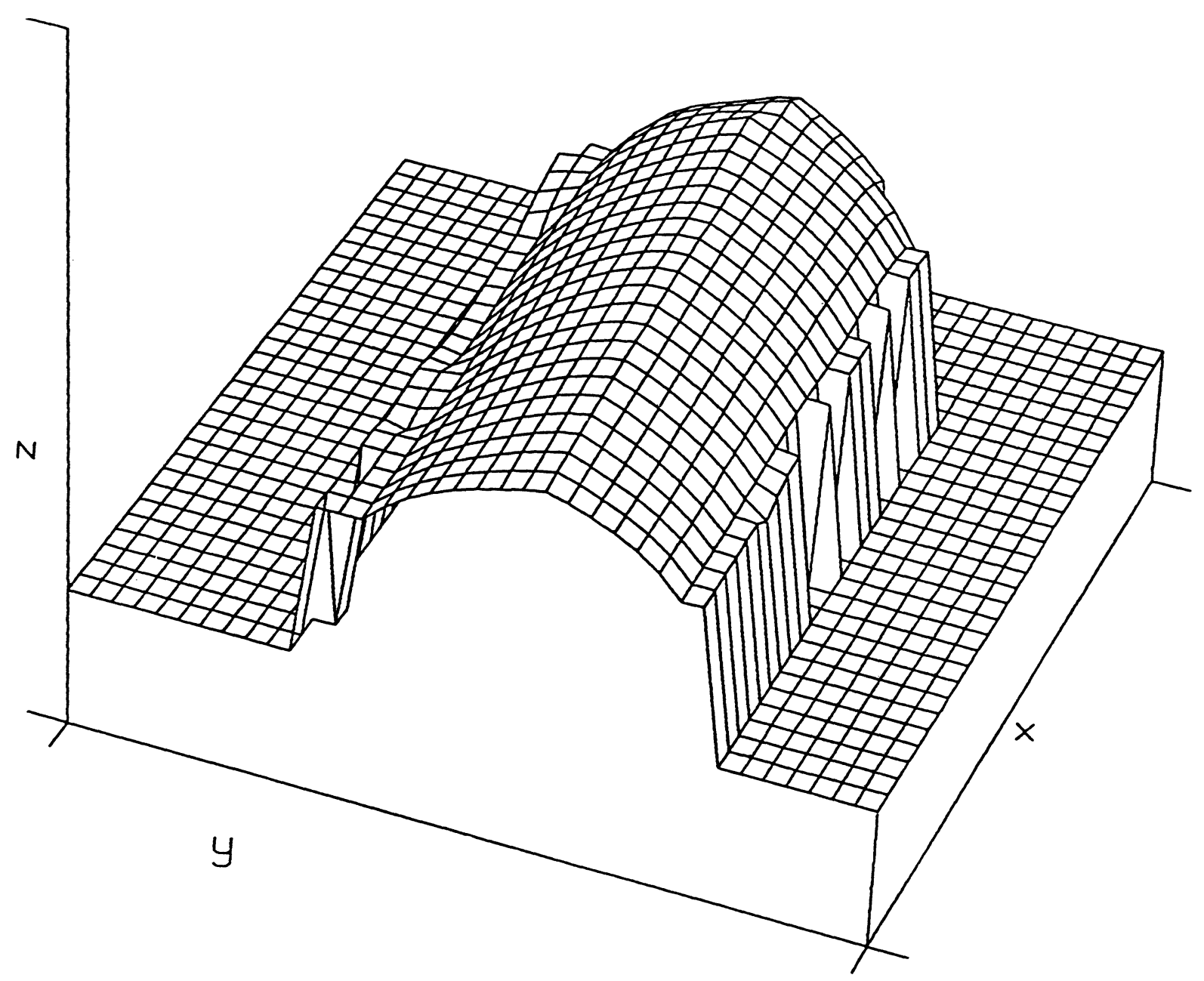

Figure 4.12 Reconstruction of the cylinder. 


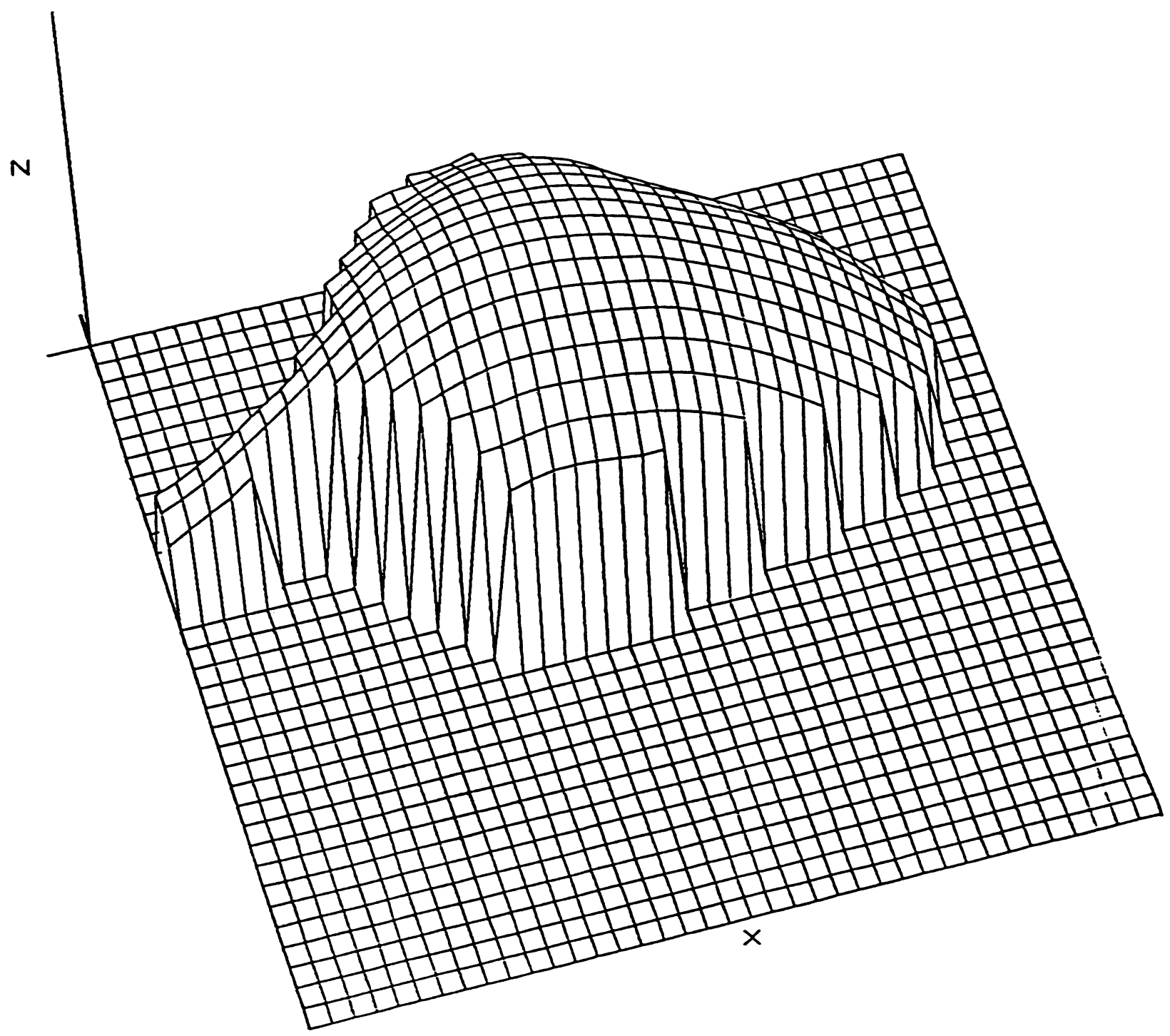

Figure 4.13 Reconstruction of the spoon. 
show the reconstruction of these objects using three light sources placed at a constant zenith angle of $25^{\circ}$ and azimuth angles of $0^{\circ}, 120^{\circ},-120^{\circ}$. Only the front part of the bottle was used in order to simulate the reconstruction of a cylinder. 


\section{Chapter 5}

\section{The Un-normalized Photometric Stereo Equation}

\subsection{Introduction}

In this chapter, we will consider the un-normalized photometric stereo equation (2.7). We will assume that we are dealing with isotropic reflectance maps. The work in this chapter is motivated by Coleman and Jain's [15] and Ray et. al.'s [42] work where the un-normalized photometric stereo problem was formulated for the Lambertian reflectance map. The main result of that work was that three light sources were sufficient for a globally unique solution for the un-normalized equation. This result is obvious since the un-normalized photometric stereo equation reduces to a linear equation for a Lambertian reflectance map. This chapter explores the possibility of inverting the un-normalized equation for non-Lambertian maps using only three light sources. Note that the un-normalized equation has three unknowns - two unknowns in $\mathbf{n}$ ( $\mathbf{n}$ is constrained by $\mathbf{n}^{T} \mathbf{n}=1$ ), and the factor $a$ in equation ( 2.7). Thus a globally unique solution with three light sources is a possibility.

The normalized photometric stereo equation gives rise to a manifold structure in the intensity space. Questions about inversion of the un-normalized photometric stereo equation can be posed as questions of the geometry of this manifold. The manifold structure of the normalized equation is explained in section 5.2. In section 5.3, a differential geometric approach is used to obtain a computationally inexpensive algorithm to check if a given reflectance map and light source placements yield a unique solution. In section 5.4 we generalize Coleman and Jain [15] and Ray [42] et. al.'s results and show that for a single-lobed non-Lambertian map, three light sources are sufficient to obtain a globally unique solution.

\subsection{The manifold structure}

If the three light sources are placed such that the normalized photometric stereo equation (2.8) has a globally unique solution then, it can be viewed as a mapping 
from $E^{3}$ to $E^{3}$, i.e., from $\mathrm{n}$ to $\left(I_{1}^{\text {rel }} I_{2}^{r e l} I_{3}^{r e l}\right)^{T}$. The domain of this mapping is the manifold $\mathbf{n}^{T} \mathbf{n}=1$ to which $\mathbf{n}$ belongs. If we assume a unique solution for the normalized equation, the range of the mapping also becomes a manifold in $E^{3}$. We denote this manifold by $S$.

Example: The manifold $S$ is best illustrated in the case of a Lambertian surface. We have,

$$
H=\left(\begin{array}{c}
\mathrm{i}_{1}^{T} \\
\mathrm{i}_{2}^{T} \\
\mathrm{i}_{3}^{T}
\end{array}\right), \quad I^{\text {rel }}=\left(\begin{array}{c}
I_{1}^{\text {rel }} \\
I_{2}^{\text {rel }} \\
I_{3}^{\text {rel }}
\end{array}\right)
$$

where, $I^{r e l}=H \mathbf{n}$. Hence, $\mathbf{n}=H^{-1} I^{r e l}$ and using $\mathbf{n}^{T} \mathbf{n}=1$ we get

$$
I^{r e l ~} T\left(H^{-1}\right)^{T}\left(H^{-1}\right) I^{r e l}=1
$$

Thus, the manifold corresponding to a Lambertian reflectance map is a part of the ellipsoid (5.1). In passing, note that equation (5.1) can be used as a test for the Lambertian-ness of a surface.

Consider the shape of the manifold $S$ when we assume that the term $\sum_{j} \rho_{j} \phi_{j}\left(\mathbf{p}_{l j}^{T} \mathbf{n}\right)$ in the m-lobed reflectance map of equation (3.21) goes to zero as $\mathbf{i}_{l}^{T} \mathbf{n}$ approaches zero. As $\mathbf{i}_{1}^{T} \mathbf{n} \rightarrow 0, I_{1}^{r e l} \rightarrow b$, i.e., the edge of the manifold corresponding to $\mathbf{i}_{1}^{T} \mathbf{n} \rightarrow 0$ is a curve in the plane $I_{1}^{r e l}=b$. The edge of the manifold corresponding to $\mathbf{i}_{2}^{T} \mathbf{n} \rightarrow 0$ is a curve in the plane $I_{2}^{r e l}=b$ and the edge corresponding to $\mathbf{i}_{3}^{T} \mathbf{n} \rightarrow 0$ is a curve in the the plane $I_{3}^{r e l}=b$. The manifold $S$ can be visualized as in figure 5.1.

Note that given any two points $\mathrm{x}_{1}$ and $\mathrm{x}_{2}$ on $S$, the plane $\Gamma$ defined by $\Gamma=$ span $\left(\mathbf{x}_{1}, \mathbf{x}_{2}\right)$ will intersect $S$ in such a fashion that it is possible to draw a continuous curve $\alpha(s)$ (see figure 5.2) with $\alpha(0)=\mathrm{x}_{1}$ and $\alpha(1)=\mathrm{x}_{2}$ and $\alpha(s) \in \Gamma \cap S$.

\subsection{The geometry of the manifold $S$}

It is easier to analyze the un-normalized equation in terms of zenith and azimuth angles rather than in terms of unit vectors. Let the normal vector $\mathbf{n}$ be denoted by the zenith and azimuth angle pair $\theta_{n}, \phi_{n}$. Let the light source direction $\mathbf{i}_{l}$ be 

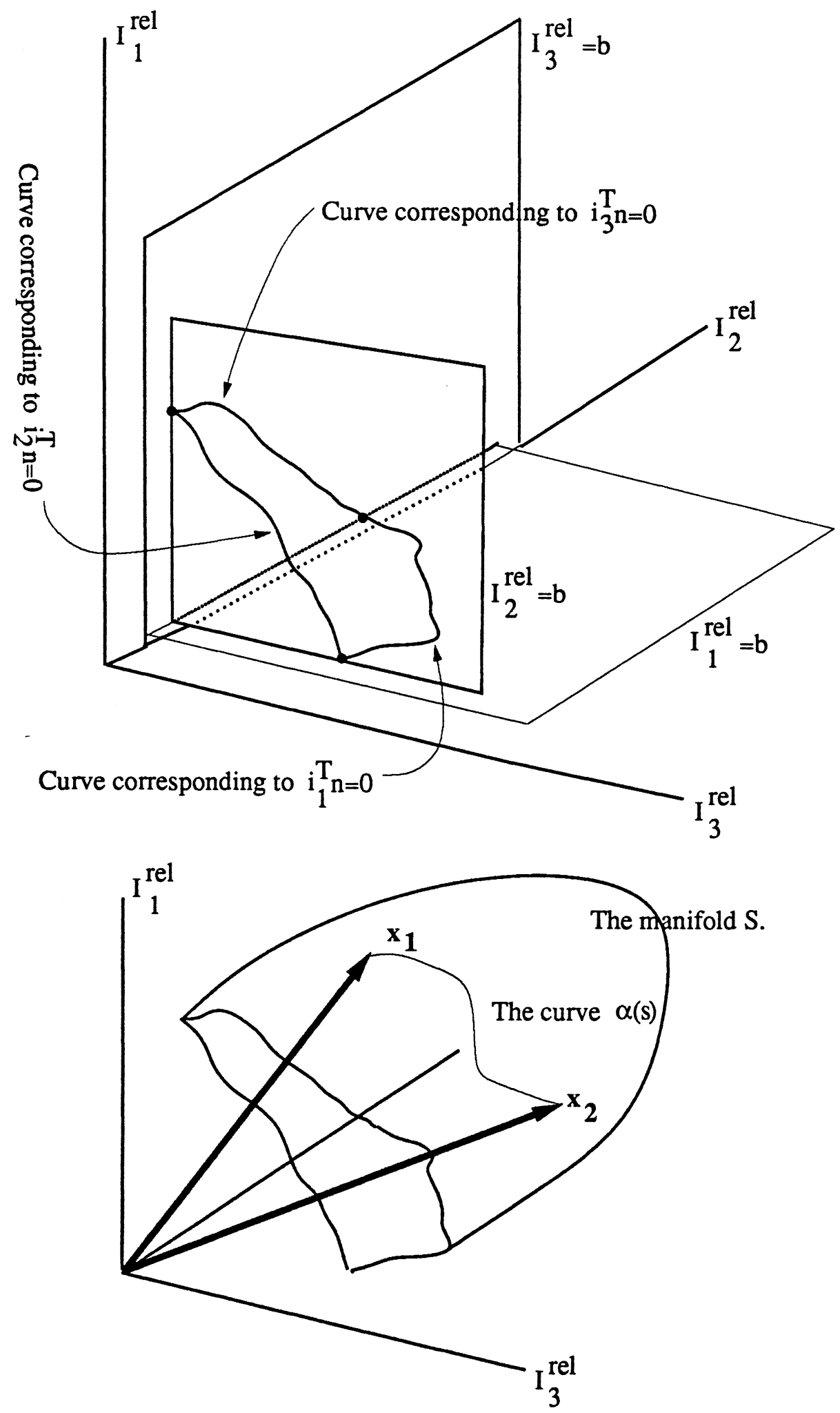

Figure 5.1 The shape of the manifold $S$ 


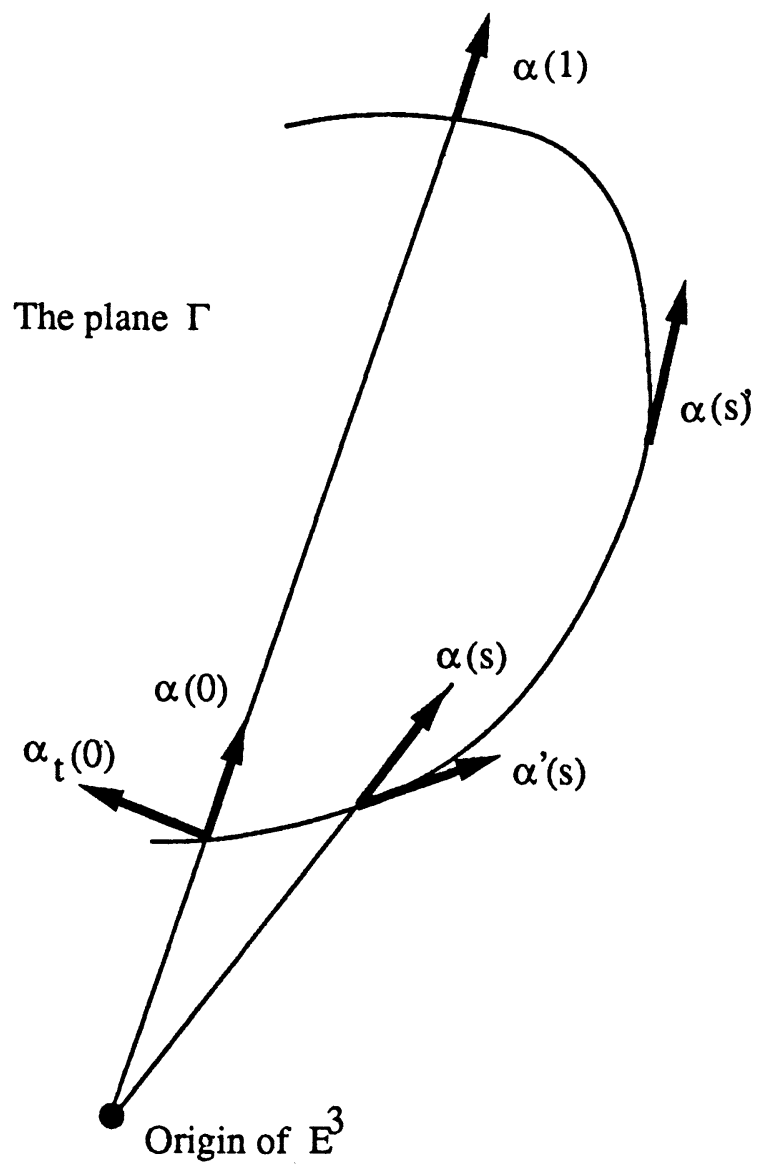

Figure 5.2 The intersection of $S$ and $\Gamma$ 
denoted by $\theta_{l}^{i}, \phi_{l}^{i}$ in which the superscript $i$ denotes that a light source is associated with this direction. In terms of these angles, the inner product is given by $\mathbf{i}_{l}^{T} \mathbf{n}=$ $\cos \theta_{l}^{i} \cos \theta_{n}+\sin \theta_{l}^{i} \sin \theta_{n} \cos \left(\phi_{l}^{i}-\phi_{n}\right)$. Let us denote by $C$ the region,

$$
\begin{aligned}
C= & \left\{\theta_{n}, \phi_{n}: \cos \theta_{1}^{i} \cos \theta_{n}+\sin \theta_{1}^{i} \sin \theta_{n} \cos \left(\phi_{1}^{i}-\phi_{n}\right)>0,\right. \\
& \cos \theta_{2}^{i} \cos \theta_{n}+\sin \theta_{2}^{i} \sin \theta_{n} \cos \left(\phi_{2}^{i}-\phi_{n}\right)>0, \\
& \left.\cos \theta_{3}^{i} \cos \theta_{n}+\sin \theta_{3}^{i} \sin \theta_{n} \cos \left(\phi_{3}^{i}-\phi_{n}\right)>0\right\} .
\end{aligned}
$$

Given a normal vector $\mathbf{n}$ whose representation is $\theta_{n}, \phi_{n} \in C$, we can write the normalized photometric stereo equation as

$$
\left(\begin{array}{c}
I_{1}^{r e l} \\
I_{2}^{r e l} \\
I_{3}^{r e l}
\end{array}\right)=\left(\begin{array}{c}
R_{1}\left(\theta_{n}, \phi_{n}\right) \\
R_{2}\left(\theta_{n}, \phi_{n}\right) \\
R_{3}\left(\theta_{n}, \phi_{n}\right)
\end{array}\right)
$$

where,

$$
\begin{aligned}
& R_{1}\left(\theta_{n}, \phi_{n}\right)=R\left(\mathbf{i}_{1}, \mathbf{n}, \mathbf{r}\right), \\
& R_{2}\left(\theta_{n}, \phi_{n}\right)=R\left(\mathbf{i}_{2}, \mathbf{n}, \mathbf{r}\right), \\
& R_{3}\left(\theta_{n}, \phi_{n}\right)=R\left(\mathbf{i}_{3}, \mathbf{n}, \mathbf{r}\right) .
\end{aligned}
$$

If we assume that the three light source directions have been chosen such that the normalized photometric stereo equation has a globally unique solution, then

$$
\operatorname{rank}\left(\begin{array}{ll}
\frac{\partial R_{1}}{\partial \theta_{n}} & \frac{\partial R_{1}}{\partial \phi_{n}} \\
\frac{\partial R_{2}}{\partial \theta_{n}} & \frac{\partial R_{2}}{\partial \phi_{n}} \\
\frac{\partial R_{3}}{\partial \theta_{n}} & \frac{\partial R_{3}}{\partial \phi_{n}}
\end{array}\right)=2
$$

Now consider the un-normalized photometric stereo equation. If it is possible to find two distinct pairs $a_{1}, \mathbf{n}_{1}$ and $a_{2}, \mathbf{n}_{2}$ such that

$$
a_{1}\left(\begin{array}{l}
R\left(\mathbf{i}_{1}, \mathbf{n}_{1}, \mathbf{r}\right) \\
R\left(\mathbf{i}_{2}, \mathbf{n}_{1}, \mathbf{r}\right) \\
R\left(\mathbf{i}_{3}, \mathbf{n}_{1}, \mathbf{r}\right)
\end{array}\right)=a_{2}\left(\begin{array}{l}
R\left(\mathbf{i}_{1}, \mathbf{n}_{2}, \mathbf{r}\right) \\
R\left(\mathbf{i}_{2}, \mathbf{n}_{2}, \mathbf{r}\right) \\
R\left(\mathbf{i}_{3}, \mathbf{n}_{2}, \mathbf{r}\right)
\end{array}\right)
$$

then,

$$
\left(\begin{array}{l}
R_{1}\left(\theta_{n_{1}}, \phi_{n_{1}}\right) \\
R_{2}\left(\theta_{n_{1}}, \phi_{n_{1}}\right) \\
R_{3}\left(\theta_{n_{1}}, \phi_{n_{1}}\right)
\end{array}\right)=\beta\left(\begin{array}{l}
R_{1}\left(\theta_{n_{2}}, \phi_{n_{2}}\right) \\
R_{2}\left(\theta_{n_{2}}, \phi_{n_{2}}\right) \\
R_{3}\left(\theta_{n_{2}}, \phi_{n_{2}}\right)
\end{array}\right),
$$


where, $\beta=a_{1} / a_{2}>0$.

We can interpret this condition in terms of the manifold $S:$ if the un-normalized photometric stereo equation does not have a globally unique solution then, there must be two elements of $S$ which are linearly dependent with a positive scaling factor. Since $S$ is a smooth manifold, the linearly dependency can be checked for by a local condition as given in the following theorem.

Theorem 5.1 If equation (5.3) holds for some distinct $\theta_{n_{1}}, \phi_{n_{1}} \in C$, $\theta_{n_{2}}, \phi_{n_{2}} \in C$, and $\beta>0$, then there is at least one element $\theta_{n}^{*}, \phi_{n}^{*} \in C$ such that,

$$
J=\operatorname{det}\left(\begin{array}{c}
R_{1}\left(\theta_{n}, \phi_{n}\right) R_{2}\left(\theta_{n}, \phi_{n}\right) R_{3}\left(\theta_{n}, \phi_{n}\right) \\
\frac{\partial R_{1}\left(\theta_{n}, \phi_{n}\right)}{\partial \theta_{n}} \frac{\partial R_{2}\left(\theta_{n}, \phi_{n}\right)}{\partial \theta_{n}} \frac{\partial R_{3}\left(\theta_{n}, \phi_{n}\right)}{\partial \theta_{n}} \\
\frac{\partial R_{1}\left(\theta_{n}, \phi_{n}\right)}{\partial \phi_{n}} \frac{\partial R_{2}\left(\theta_{n}, \phi_{n}\right)}{\partial \phi_{n}} \frac{\partial R_{3}\left(\theta_{n}, \phi_{n}\right)}{\partial \phi_{n}}
\end{array}\right)_{\theta_{n}=\theta_{n}^{*}, \phi_{n}=\phi_{n}^{*}}=0 .
$$

Proof Let us interpret equation (5.4) geometrically. Given $\theta_{n}, \phi_{n} \in C$, consider the space $E^{3}$ from the point $I^{r e l}\left(\theta_{n}, \phi_{n}\right)$ on $S$. The origin of the space $E^{3}$ with respect to this point is given by $-I^{\text {rel }}\left(\theta_{n}, \phi_{n}\right)$. The tangent plane to $S$ at the point is spanned by

$$
\left(\begin{array}{l}
\frac{\partial R_{1}}{\partial \theta} \\
\frac{\partial R_{2}}{\partial \theta} \\
\frac{\partial R_{3}}{\partial \theta}
\end{array}\right) \text { and }\left(\begin{array}{c}
\frac{\partial R_{1}}{\partial \phi} \\
\frac{\partial R_{2}}{\partial \phi} \\
\frac{\partial R_{3}}{\partial \phi}
\end{array}\right)
$$

Condition (5.4) states that the vector $I^{\text {rel }}\left(\theta_{n}^{*}, \phi_{n}^{*}\right)$, and consequently $-I^{r e l}\left(\theta_{n}^{*}, \phi_{n}^{*}\right)$, lie in the tangent plane of $S$ at $I^{\text {rel }}\left(\theta_{n}^{*}, \phi_{n}^{*}\right)$. Thus, 5.4 states that there is at least one point on the manifold $S$ at which the tangent plane includes the origin of $E^{3}$.

Note that if equation (5.3) holds for some $\theta_{n_{1}}, \phi_{n_{1}}$ and $\theta_{n_{2}}, \phi_{n_{2}}$, then there is at least one $\theta_{n_{3}}, \phi_{n_{3}} \in C$ for which it does not, i.e., $I^{r e l}\left(\theta_{n_{3}}, \phi_{n_{3}}\right) \neq \nu I^{\text {rel }}\left(\theta_{n 1}, \phi_{n_{1}}\right)$, for any $\nu>0$. If this were not so, all the points of $S$ would lie along the vector $I^{\text {rel }}\left(\theta_{1}, \phi_{1}\right)$ and (5.2) would not hold. Given such a $\theta_{n_{3}}, \phi_{n_{3}}$ we can construct a plane (call it $\Gamma$ ) which is the span of $I^{\text {rel }}\left(\theta_{n_{1}}, \phi_{n_{1}}\right)$ and $I^{\text {rel }}\left(\theta_{n_{3}}, \phi_{n_{3}}\right)$. Note that since $I^{\text {rel }}\left(\theta_{n_{2}}, \phi_{n_{2}}\right)=$ $\beta I^{r e l}\left(\theta_{n_{1}}, \phi_{n_{1}}\right), I^{\text {rel }}\left(\theta_{n_{2}}, \phi_{n_{2}}\right) \in \Gamma$. Hence, the points $I^{\text {rel }}\left(\theta_{n_{1}}, \phi_{n_{1}}\right)$ and $I^{\text {rel }}\left(\theta_{n_{2}}, \phi_{n_{2}}\right)$ are contained both in $S$ and $\Gamma$.

Consider the intersection of $S$ and $\Gamma$. From our discussion in section 5.2 it is clear this intersection will contain at least one continuous curve $\alpha(s)$, such that $\alpha(0)=$ $I^{\text {rel }}\left(\theta_{n_{1}}, \phi_{n_{1}}\right)$ and $\alpha(1)=I^{\text {rel }}\left(\theta_{n_{2}}, \phi_{n_{2}}\right)$. Since the curve is continuous, it has well defined tangent vectors $\alpha^{\prime}(s)$ everywhere. 
We will now proceed to show that there is at least one point on the curve $\alpha(s)$, at which $\alpha^{\prime}(s)$ becomes linearly dependent on $\alpha(s)$. Since, $-\alpha(s)$ is in fact the origin of $E^{3}$ and $\alpha^{\prime}(s)$ is contained in the tangent space of $S$, at $\alpha(s)$ we have found the point we are looking for and the theorem is proved.

As $\Gamma$ is a plane, given any vector $\alpha(s)$ in it, we can construct another vector $\alpha_{t}(s) \in \Gamma$ such that $\|\alpha(s)\|=1, \alpha_{t}^{T}(s) \alpha(s)=0$, and the functions

$$
f(s)=\alpha_{t}^{T}(0) \alpha(s), \quad g(s)=\alpha_{t}^{T}(s) \alpha^{\prime}(s)
$$

are well defined and continuous (see figure 5.2). Now, $f(0)=0$ and $f(1)=\alpha_{t}^{T}(0) \alpha(1)=$ $\beta \alpha_{t}^{T}(0) \alpha(0)=0$. Since $f^{\prime}(s)=\alpha_{t}^{T}(0) \alpha^{\prime}(s)$, we have the following possibilities for $f^{\prime}(s)$

1. $f^{\prime}(0)=0$. In this case, we get $\alpha_{t}^{T}(0) \alpha^{\prime}(0)=0$, and so $\alpha^{\prime}(0)$ is linearly dependent on $\alpha(0)$, which proves the theorem.

2. $f^{\prime}(0) \neq 0$. Let $s^{\prime}$ be a point $0<s^{\prime} \leq 1$, such that $f\left(s^{\prime}\right)=0$ and $f(s) \neq 0$ for all $s, 0<s<s^{\prime}$. We know that there is at least one such point given by $s^{\prime}=1$ (see figure 5.2). Since $f\left(s^{\prime}\right)=\alpha_{t}^{T}(0) \alpha\left(s^{\prime}\right)=0$, we have that $\alpha\left(s^{\prime}\right)$ has no component along $\alpha_{t}(0)$, and hence, $\alpha\left(s^{\prime}\right)$ is linearly dependent on $\alpha(0)$. This implies that, $\alpha_{t}\left(s^{\prime}\right)=\alpha_{t}(0)$. At $s^{\prime}$ we have two possibilities

(a) $f^{\prime}\left(s^{\prime}\right)=0$. This again implies that the tangent vector $\alpha^{\prime}\left(s^{\prime}\right)$ and the vector $\alpha\left(s^{\prime}\right)$ are linearly dependent and the theorem follows.

(b) $f^{\prime}\left(s^{\prime}\right) \neq 0$. Then, $\operatorname{sign} f^{\prime}\left(s^{\prime}\right)=-\operatorname{sign} f^{\prime}(0)$. We have, $g\left(s^{\prime}\right)=\alpha_{t}^{T}\left(s^{\prime}\right) \alpha^{\prime}\left(s^{\prime}\right)=$ $\alpha_{t}^{T}(0) \alpha^{\prime}\left(s^{\prime}\right)=f^{\prime}\left(s^{\prime}\right)$. Also, $g(0)=\alpha_{t}^{T}(0) \alpha^{\prime}(0)=f^{\prime}(0)$. Thus, sign $g(0)=$ - sign $g(1)$ and there must exist an $s^{*}, 0 \leq s^{*} \leq 1$, such that $g\left(s^{*}\right)=0$. This implies that at $s^{*}$, the vectors $\alpha\left(s^{*}\right)$ and $\alpha^{\prime}\left(s^{*}\right)$ are linearly dependent and the theorem follows.

Note that the result of this theorem reduces the computational complexity of checking the global nature of the un-normalized photometric stereo equation. If the property of equation (5.3) is checked for in a naive way, it has to be evaluated for every pair of vectors $n_{1}, n_{2}$. Theorem 5.1 reduces this computation to finding a zero crossing of a function of $\mathbf{n}$. The complexity is reduced from $\mathrm{O}\left(n^{2}\right)$ to $\mathrm{O}(n)$.

Figure 5.3 shows a plot of $J$ of equation (5.4) for the non-Lambertian model of equation (3.22). The three light sources are placed at a constant zenith angle of $25^{\circ}$ 


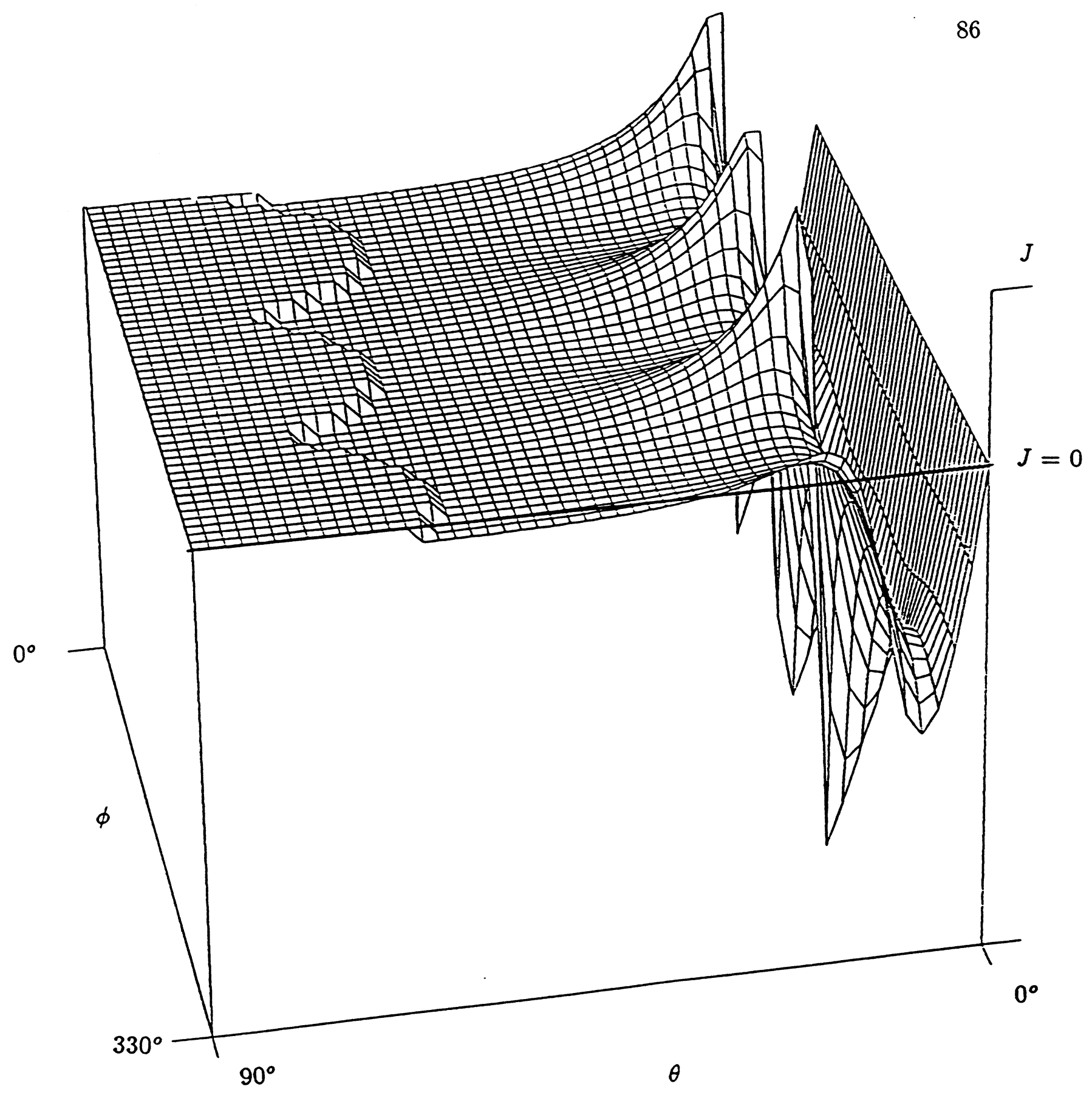

Figure 5.3 The function $J$ for the physical reflectance map 
and azimuth angles of $0^{\circ}, 120^{\circ}$, and $-120^{\circ}$. Note that the value of $J$ does cross zero. Hence, by theorem 5.1, we are faced with the possibility that the albedo and the surface normal cannot be uniquely determined for this reflectance map. In fact, it is very easy to find two surface normals that satisfy equation (5.3). Let $\theta_{n_{1}}=50.0^{\circ}$, $\theta_{n_{2}}=20.83^{\circ}$ and $\phi_{n_{1}}=\phi_{n_{2}}=0.0$. Then, we have $\left[R_{1}\left(\theta_{1}, \phi_{1}\right) R_{2}\left(\theta_{1}, \phi_{1}\right) R_{3}\left(\theta_{1}, \phi_{1}\right)\right]^{T}=$ $\left[\begin{array}{lll}0.51117 & 0.21174 & 0.21174\end{array}\right]^{T}$ and $\left[R_{1}\left(\theta_{2}, \phi_{2}\right) R_{2}\left(\theta_{2}, \phi_{2}\right) R_{3}\left(\theta_{1}, \phi_{1}\right)\right]^{T}=\left[\begin{array}{lll}1.367620 .566620 .56662\end{array}\right]^{T}$.

It is easy to very that this satisfies equation (5.3) with $\beta \simeq 2.6757$. Thus, for this arrangement, we cannot estimate the albedo and the surface normal uniquely by using three light sources.

At this time, it has not been possible to explore this manifold structure further. The problem is two-fold:

1. The normalized photometric stereo equation can be viewed as an extrinsic specification of the manifold $S$. In order to compute the properties of the manifold, this extrinsic description has to be turned into an intrinsic one, so that the resulting description of the manifold is independent of $\mathbf{n}$. The only known procedures for converting an extrinsic description to an intrinsic one are for polynomial surfaces [45]. The Lambertian reflectance map is linear and for this map, as we saw in the example above, it is possible to get an intrinsic description for the manifold fairly easily. In general, for non-polynomial surfaces this cannot be done.

2. Increasing the number of light sources increases the dimension of the space the manifold $S$ is embedded in while retaining the locally $E^{2}$ structure of the manifold. Now, we can no longer assert the property of section 5.2 that given any two points $\mathbf{x}_{1}$ and $\mathbf{x}_{2}$ on $S$, the hyperplane $\Gamma$ defined by $\Gamma=\operatorname{span}\left(\mathbf{x}_{1}, \mathbf{x}_{2}\right)$ will intersect $S$ in such a fashion that it is possible to draw a continuous curve $\alpha(s)$ with $\alpha(0)=\mathrm{x}_{1}$ and $\alpha(1)=\mathrm{x}_{2}$ and $\alpha(s) \in \Gamma \cap S$.

However, as we shall in the next section, it is possible to investigate some reflectance maps without explicitly writing down the intrinsic equation for the manifold.

\subsection{Single-lobed map}

In this section, we investigate the behavior of the un-normalized photometric stereo equation in the case of a single-lobed map. For the physical reflectance maps whose 
forescatter lobe width is small, it is possible to use a number of light sources such that for any surface normal, at least three of the light sources reflect light into the camera without a significant forescatter component [39]. The intensities due to these light sources are used to estimate the Lambertian component which can be subtracted out from all the intensity values. The remaining intensities can be used to estimate the albedo of the forescatter lobe. Coleman and Jain [15] used this approach first on surfaces that reflected purely in a Lambertian-specular manner. Nayar, Kanade and Ikeuchi [39] extended the approach for a Lambertian-specular surface illuminated with extended light sources. In their study, after estimating the Lambertian, they estimate the albedo of the remaining single lobed map using a heuristic procedure. They do not prove that the answer is unique or that the number of light sources they use is minimum. Clearly, a theorem of uniqueness of solution to the un-normalized photometric stereo equation for the single-lobed map would put such techniques on firmer ground.

Consider the un-normalized photometric stereo equation for a single-lobed map without backscatter. The un-normalized equation reduces to

$$
\left(\begin{array}{l}
I_{1}(\mathbf{n}) \\
I_{2}(\mathbf{n}) \\
I_{3}(\mathbf{n})
\end{array}\right)=a\left(\begin{array}{l}
\phi\left(\mathbf{p}_{11}^{T} \mathbf{n}\right) \\
\phi\left(\mathbf{p}_{21}^{T} \mathbf{n}\right) \\
\phi\left(\mathbf{p}_{31}^{T} \mathbf{n}\right)
\end{array}\right)
$$

where, $\phi()$ is a monotone, convex and log-concave function and $\mathbf{p}_{l 1}$ is the first principle direction associated with the light source illuminating from the direction $\mathbf{i}_{l}$. If the normalized equation does not have a globally unique solution, then, for some $\mathbf{n}_{1}$ and $\mathbf{n}_{2}$

$$
\left(\begin{array}{c}
\phi\left(\mathbf{p}_{11}^{T} \mathrm{n}_{1}\right) \\
\phi\left(\mathbf{p}_{21}^{T} \mathbf{n}_{1}\right) \\
\phi\left(\mathbf{p}_{31}^{T} \mathbf{n}_{1}\right)
\end{array}\right)=\beta\left(\begin{array}{c}
\phi\left(\mathbf{p}_{11}^{T} \mathrm{n}_{2}\right) \\
\phi\left(\mathbf{p}_{21}^{T} \mathbf{n}_{2}\right) \\
\phi\left(\mathbf{p}_{31}^{T} \mathbf{n}_{2}\right)
\end{array}\right)
$$

and,

$$
\left(\begin{array}{c}
\log \phi\left(\mathbf{p}_{11}^{T} \mathbf{n}_{1}\right) \\
\log \phi\left(\mathbf{p}_{21}^{T} \mathbf{n}_{1}\right) \\
\log \phi\left(\mathbf{p}_{31}^{T} \mathbf{n}_{1}\right)
\end{array}\right)=\left(\begin{array}{c}
\log \phi\left(\mathbf{p}_{11}^{T} \mathbf{n}_{2}\right) \\
\log \phi\left(\mathbf{p}_{21}^{T} \mathbf{n}_{2}\right) \\
\log \phi\left(\mathbf{p}_{31}^{T} \mathbf{n}_{2}\right)
\end{array}\right)+\log \beta\left(\begin{array}{l}
1 \\
1 \\
1
\end{array}\right) .
$$

Let, $\eta()=\log \phi()$. Since $\log ()$ and $\phi()$ are monotone strictly increasing functions, so is $\eta()$. Let $\zeta()=\eta^{-1}()$. Then, $\zeta()$ is a monotone, strictly increasing function. 
Also, since since $\phi()$ is log-concave, $\eta()$ is concave and $\zeta()$ is convex. Let us denote the manifold generated in $E^{3}$ by

$$
\left(\begin{array}{l}
\log \phi\left(\mathbf{p}_{11}^{T} \mathbf{n}\right) \\
\log \phi\left(\mathbf{p}_{21}^{T} \mathbf{n}\right) \\
\log \phi\left(\mathbf{p}_{31}^{T} \mathbf{n}\right)
\end{array}\right)
$$

as $S_{1}$.

If equation (5.5) holds, then the manifold $S_{1}$ has the property that the line

$$
t\left(\begin{array}{l}
1 \\
1 \\
1
\end{array}\right)+\left(\begin{array}{l}
\gamma \\
\delta \\
\epsilon
\end{array}\right), \quad t \geq 0
$$

intersects $S_{1}$ at two different places for some positive values of $\gamma, \delta$ and $\epsilon$.

Now, consider the following mapping $\Gamma(): E^{3} \rightarrow E^{3}$

$$
\Gamma\left(\begin{array}{l}
x_{1} \\
x_{2} \\
x_{3}
\end{array}\right)=\left(\begin{array}{l}
\zeta\left(x_{1}\right) \\
\zeta\left(x_{2}\right) \\
\zeta\left(x_{3}\right)
\end{array}\right), \quad x_{1}, x_{2}, x_{3}>0,
$$

where, $\zeta()$ is the function defined above. Since $\zeta()$ is $1-1$, the mapping $\Gamma()$ maps $S_{1}$ into another manifold $S$ and the line of equation (5.6) into a curve $\alpha(t), t>0$ given by

$$
\alpha(t)=\left(\begin{array}{l}
\zeta(t+\gamma) \\
\zeta(t+\delta) \\
\zeta(t+\epsilon)
\end{array}\right)
$$

If the manifold $S_{1}$ was such that the line of equation (5.6) intersected it twice, then the curve of equation (5.8) would intersect the manifold $S$ twice too. The following theorem shows that for a symmetric light placement this cannot happen.

Theorem 5.2 If three light sources are placed at the same azimuth angle $\theta_{i}\left(\pi / 2>\theta_{i}>0\right)$ and at azimuth angles of $0^{\circ}, 120^{\circ}$ and $-120^{\circ}$, then the curve $\alpha(t)$ of equation (5.8) intersects the manifold $S$ (as defined above) only once.

Proof Since the reflectance map is assumed to be isotropic, the principle directions $\mathrm{p}_{l 1}$ associated with the light sources for the geometry of this theorem have a constant 
zenith angle (call it $\theta$ ) and azimuth angles of $0^{\circ}, 120^{\circ}$ and $-120^{\circ}$. The matrix $H$ is given by

$$
H=\left(\begin{array}{ccc}
\sin \theta & 0 & \cos \theta \\
-\frac{1}{2} \sin \theta & \frac{\sqrt{3}}{2} \sin \theta & \cos \theta \\
-\frac{1}{2} \sin \theta & -\frac{\sqrt{3}}{2} \sin \theta & \cos \theta
\end{array}\right)
$$

and the points on the manifold $S$ are given by $H \mathbf{n}$, where $\mathbf{n}$ is constrained as $\mathbf{n}^{T} \mathbf{n}=1$, i.e. the manifold is an ellipsoid.

1. The structure of the ellipsoid $S$ is given simply as follows. Let $\mathbf{v}_{1}, \mathbf{v}_{2}, \mathbf{v}_{3}$ be the eigenvectors of $H^{T} H$ and $\lambda_{1}, \lambda_{2}, \lambda_{3}$ be the corresponding eigenvalues. The axis of the ellipsoid are along the vectors:

$$
\begin{aligned}
& \mathbf{w}_{\mathbf{1}}=\frac{H \mathbf{v}_{1}}{\left\|H \mathbf{v}_{1}\right\|}=\frac{1}{\sqrt{3}}\left(\begin{array}{l}
1 \\
1 \\
1
\end{array}\right), \\
& \mathbf{w}_{\mathbf{2}}=\frac{H \mathbf{v}_{2}}{\left\|H \mathbf{v}_{2}\right\|}=\frac{1}{\sqrt{3}}\left(\begin{array}{c}
1 \\
-1 / 2 \\
-1 / 2
\end{array}\right), \\
& \mathbf{w}_{\mathbf{3}}=\frac{H \mathbf{v}_{3}}{\left\|H \mathbf{v}_{3}\right\|}=\frac{1}{\sqrt{2}}\left(\begin{array}{c}
0 \\
1 \\
-1
\end{array}\right),
\end{aligned}
$$

and,

$$
\begin{aligned}
& \lambda_{1}=3 \cos \theta \\
& \lambda_{2}=3 / 2 \sin ^{2} \theta \\
& \lambda_{3}=3 / 2 \sin ^{2} \theta
\end{aligned}
$$

Note that $\lambda_{2}=\lambda_{3}$. Further, let us define a vector

$$
\mathbf{u}_{2}=\frac{1}{\sqrt{2}}\left(\begin{array}{c}
1 \\
-1 \\
0
\end{array}\right) \text {. }
$$

2. The ellipsoid induces a norm on the space. Given a vector $\mathrm{x} \in E^{3}$, this norm is

$$
\|\mathbf{x}\|_{S}^{2}=\frac{1}{\lambda_{1}^{2}}\left(\mathbf{x}^{T} \mathbf{w}_{1}\right)^{2}+\frac{1}{\lambda_{2}^{2}}\left(\mathbf{x}^{T} \mathbf{w}_{2}\right)^{2}+\frac{1}{\lambda_{3}^{2}}\left(\mathbf{x}^{T} \mathbf{w}_{3}\right)^{2} .
$$

Note that if $\mathbf{x} \in S$, then $\|\mathrm{x}\|_{S}^{2}=1$. 
Our proof consists of showing that $\|\alpha(t)\|_{S}^{2}$ is a strictly increasing function of $t$. Hence, $\|\alpha(t)\|_{S}^{2}$ can achieve equality with 1 only once and can intersect $S$ only once.

$$
\begin{aligned}
\|\alpha(t)\|_{S}^{2}= & \frac{1}{\lambda_{1}^{2}}\left(\alpha(t)^{T} \mathbf{w}_{1}\right)^{2} \\
& +\frac{1}{\lambda_{2}^{2}}\left(\alpha(t)^{T} \mathbf{w}_{2}\right)^{2}+\frac{1}{\lambda_{3}^{2}}\left(\alpha(t)^{T} \mathbf{w}_{3}\right)^{2} \\
= & \frac{1}{\lambda_{1}^{2}}\left(\alpha(t)^{T} \mathbf{w}_{1}\right)^{2} \\
& +\frac{1}{\lambda_{2}^{2}}\left\{\left(\alpha(t)^{T} \mathbf{w}_{2}\right)^{2}+\left(\alpha(t)^{T} \mathbf{w}_{3}\right)^{2}\right\} \\
= & \frac{1}{\lambda_{1}^{2}}\left(\alpha(t)^{T} \mathbf{w}_{1}\right)^{2} \\
& +\frac{1}{\lambda_{2}^{2}} \frac{4}{3}\left\{\left(\alpha(t)^{T} \mathbf{u}_{2}\right)^{2}+2\left(\alpha(t)^{T} \mathbf{w}_{3}\right)^{2}\right\} \\
= & \frac{1}{\lambda_{1}^{2}}(\zeta(t+\gamma)+\zeta(t+\delta)+\zeta(t+\epsilon))^{2} \\
& +\frac{1}{\lambda_{2}^{2}} \frac{4}{3}\left\{(\zeta(t+\gamma)-\zeta(t+\delta))^{2}+2(\zeta(t+\delta)-\zeta(t+\epsilon))^{2}\right\}
\end{aligned}
$$

Now, since $\zeta()$ is a monotone, strictly increasing function the first term in the above expression is a strictly increasing function of $t$. Also, since $\zeta()$ is convex, the terms $\zeta(t+\gamma)-\zeta(t+\delta)$ and $\zeta(t+\delta)-\zeta(t+\epsilon)$ are either non-increasing or non-decreasing [18]. Hence, the second term in the above expression is non-decreasing and the entire expression is a strictly increasing function of $t$.

We have established that for the single lobe map the un-normalized photometric stereo equation can be solved in a globally unique fashion by using a symmetric placement of lights. 


\section{Chapter 6}

\section{Joint estimation of surface normals and reflectance map}

\subsection{Introduction}

In this chapter, we will address the problem of joint estimation of surface normals and some of the parameters of the reflectance map. Substituting the physical reflectance map of equation (3.19) (without the backscatter term) into the un-normalized photometric stereo equation (2.7) we have

$$
\begin{aligned}
\left(\begin{array}{c}
I_{1}\left(\mathbf{i}_{1}, \mathbf{n}, \mathbf{r}\right) \\
\vdots \\
I_{1}\left(\mathbf{i}_{k}, \mathbf{n}, \mathbf{r}\right)
\end{array}\right) & =a\left(\begin{array}{c}
R\left(\mathbf{i}_{1}, \mathbf{n}, \mathbf{r}\right) \\
\vdots \\
R\left(\mathbf{i}_{k}, \mathbf{n}, \mathbf{r}\right)
\end{array}\right) \\
& =a \rho_{f s c}\left(\begin{array}{c}
\exp \left(-c \cos ^{-1} \mathbf{n}_{\mathbf{s}_{\mathbf{1}}}{ }^{T} \mathbf{n}\right)^{2} \\
\vdots \\
\exp \left(-c \cos ^{-1} \mathbf{n}_{\mathbf{s}_{\mathbf{k}}}{ }^{T} \mathbf{n}\right)^{2}
\end{array}\right)+a \rho_{\text {norm }}\left(\begin{array}{c}
\mathbf{i}_{1}^{T} \mathbf{n} \\
\vdots \\
\mathbf{i}_{k}^{T} \mathbf{n}
\end{array}\right) \\
& =a_{1}\left(\begin{array}{c}
\exp \left(-c \cos ^{-1} \mathbf{n}_{\mathbf{s}_{1}}{ }^{T} \mathbf{n}\right)^{2} \\
\vdots \\
\exp \left(-c \cos ^{-1} \mathbf{n}_{\mathbf{s}_{\mathbf{k}}}{ }^{T} \mathbf{n}\right)^{2}
\end{array}\right)+a_{2}\left(\begin{array}{c}
\mathbf{i}_{\mathbf{1}}{ }^{T} \mathbf{n} \\
\vdots \\
\mathbf{i}_{\mathbf{k}}{ }^{T} \mathbf{n}
\end{array}\right) .
\end{aligned}
$$

where, $a_{1}=a \rho_{f s c}$ and $a_{2}=a \rho_{\text {norm }}$. The problem we are interested in is the estimation of the surface normal $\mathrm{n}$ and the "albedos" $a_{1}$ and $a_{2}$ in equation (6.1). We assume that we know the value of $c$.

There has been very little published work on the problem of joint estimation for diffusely reflecting surfaces. Since most of the work on diffusely reflecting surfaces assumes that the reflection is Lambertian, the single lobed un-normalized equation is most commonly used. Silver [47] did attempt to solve the problem of joint estimation, but he did not verify his technique either experimentally or numerically. In fact, his comment on his own technique is: "We must be careful to avoid being seduced by a clever mathematical trick into believing that we have developed a practical method." 
For, specular-Lambertian surfaces, joint estimation of the surface normal and the reflectance map has been attempted with extended light sources [39]. However, as we mentioned in the previous chapter, this work assumes that one of the lobe width is narrow enough so that for some directions of incidence, the most significant contribution to the reflected light is from a single lobe. A separation of the data can then be very easily achieved and the single lobed un-normalized photometric stereo equation be used to obtain the surface normal and the albedo.

In this chapter, we will not assume that the lobe width of any of components is small to affect such a separation. An exact solution to equation (6.1) is not yet available. However, a good approximation can be obtained as shown in section 6.2. That section also also obtains the number of light sources needed to solve equation (6.1) in a globally unique fashion. Unfortunately, as we shall see in section 6.3 the problem of solving for $\mathrm{n}, a_{1}$ and $a_{2}$ can become ill-posed. In section 6.4 , we present a Tikhonov regularization of the ill-posed problem. Section 6.5 contains experimental results of applying the resulting regularized procedure to real-world images.

\subsection{An Approximate Solution}

In this section, we will solve the joint estimation problem in an approximate fashion. We will do this by first demonstrating that $\phi_{n}$ the azimuth angle of the surface normal can be estimated independently of the rest of the variables. In fact, an approximate but closed form solution can be obtained for $\phi_{n}$. We will then establish that the rest of the variables can also be estimated in a globally unique fashion.

Let us make two approximations

1. We approximate $\cos \theta$ by its Taylor series expansion $\cos \theta \cong 1-\theta^{2} / 2$. Then, $\theta^{2} \cong 2(1-\cos \theta)$, i.e., $\left(\cos ^{-1} x\right)^{2} \cong 2(1-x)$.

2. The second assumption involves approximating the function $\exp \left(-c \cos ^{-1} \mathbf{n}_{\mathbf{s}}{ }^{T} \mathbf{n}\right)^{2}$ as a band limited function of $\phi_{i}$. The details are given below.

As before, let us assume that the light sources are placed at a constant zenith angle $\theta_{i}$. Since we are using the physical model we have $\theta_{n_{s}}=\theta_{i} / 2$. Let us assume that there is no backscatter and consider how the intensity $I()$ varies as a function of $\phi_{i}$ for fixed $\theta_{i}, \phi_{n}, \theta_{n}$. We have

$$
\begin{aligned}
I\left(\phi_{i}\right)= & a_{1} \exp \left(-c \cos ^{-1} \cos \theta_{i} / 2 \cos \theta_{n}+\sin \theta_{i} / 2 \sin \theta_{n} \cos \left(\phi_{i}-\phi_{n}\right)\right)^{2} \\
& +a_{2} \cos \theta_{i} \cos \theta_{n}+a_{2} \sin \theta_{i} \sin \theta_{n} \cos \left(\phi_{i}-\phi_{n}\right)
\end{aligned}
$$


Now, consider the function $g()$ defined below

$$
g(x)=\exp (\alpha x) \quad \alpha>0
$$

The, $g\left(\cos \phi_{i}\right)=\exp \left(\alpha \cos \phi_{i}\right)$. Consider the Fourier series expansion of $g\left(\cos \phi_{i}\right)$. Since $g\left(\cos \phi_{i}\right)$ is an even function of $\phi_{i}$, we have only the cosine terms in the expansion. Let,

$$
g\left(\cos \phi_{i}\right)=a_{o}+\sum_{k=1}^{\infty} a_{k} \cos \left(k \phi_{i}\right)
$$

It can be shown that ${ }^{1}[33]$

$$
\frac{a_{k+1}}{a_{k}}=-\frac{1}{2 k+1} \frac{\int_{-1}^{1}\left(1-y^{2}\right)^{k+1 / 2} g^{(k+1)}(y) d y}{\int_{-1}^{1}\left(1-y^{2}\right)^{k-1 / 2} g^{(k)}(y) d y}
$$

where, $g^{(k)}(y)$ denotes the $k^{\text {th }}$ derivative of $g()$. Note that $g^{(k+1)}=\alpha g^{(k)}(y)>0$, and within the limits of integration $0 \leq\left(1-y^{2}\right) \leq 1$. Hence,

$$
\begin{aligned}
\left|\frac{a_{k+1}}{a_{k}}\right| & =\frac{1}{2 k+1} \frac{\left|\int_{-1}^{1}\left(1-y^{2}\right)\left(1-y^{2}\right)^{k-1 / 2} g^{(k+1)}(y) d y\right|}{\left|\int_{-1}^{1}\left(1-y^{2}\right)^{k-1 / 2} g^{(k)}(y) d y\right|} \\
& =\frac{\alpha}{2 k+1} \frac{\left|\int_{-1}^{1}\left(1-y^{2}\right)\left(1-y^{2}\right)^{k-1 / 2} g^{(k)}(y) d y\right|}{\left|\int_{-1}^{1}\left(1-y^{2}\right)^{k-1 / 2} g^{(k)}(y) d y\right|} \\
& \leq \frac{\alpha}{2 k+1} \frac{\left|\int_{-1}^{1}\left(1-y^{2}\right)^{k-1 / 2} g^{(k)}(y) d y\right|}{\left|\int_{-1}^{1}\left(1-y^{2}\right)^{k-1 / 2} g^{(k)}(y) d y\right|} \\
& =\frac{\alpha}{2 k+1} .
\end{aligned}
$$

Thus, for $k \geq 1 / 2(\alpha-1)$ the spectrum of $g\left(\cos \phi_{i}\right)$ falls off rapidly. Our approximation consists of saying that $g\left(\cos \phi_{i}\right)$ is in fact bandlimited to some harmonic. Therefore, we assume that we can sample $g\left(\cos \phi_{i}\right)$ at the Nyquist rate and retain all the information in it.

Now, using the approximation $\left(\cos ^{-1} x\right)^{2} \simeq 2(1-x)$ we can write

$$
\begin{aligned}
I\left(\phi_{i}\right)= & a_{1} \exp \left(-c \cos ^{-1} \cos \theta_{i} / 2 \cos \theta_{n}+\sin \theta_{i} / 2 \sin \theta_{n} \cos \left(\phi_{i}-\phi_{n}\right)\right)^{2} \\
& +a_{2} \cos \theta_{i} \cos \theta_{n}+a_{2} \sin \theta_{i} \sin \theta_{n} \cos \left(\phi_{i}-\phi_{n}\right) \\
\simeq & a_{1} \exp \left(-2 c^{2}+2 c^{2} \cos \theta_{i} / 2 \cos \theta_{n}+2 c^{2} \sin \theta_{i} / 2 \sin \theta_{n} \cos \left(\phi_{i}-\phi_{n}\right)\right)
\end{aligned}
$$

\footnotetext{
${ }^{1}$ I am indebted to Prof. Don. H. Johnson for providing this result.
} 


$$
\begin{aligned}
& +a_{2} \cos \theta_{i} \cos \theta_{n}+a_{2} \sin \theta_{i} \sin \theta_{n} \cos \left(\phi_{i}-\phi_{n}\right) \\
= & a_{1} \exp \left(-2 c^{2}+2 c^{2} \cos \theta_{i} / 2 \cos \theta_{n}\right) \exp \left(2 c^{2} \sin \theta_{i} / 2 \sin \theta_{n} \cos \left(\phi_{i}-\phi_{n}\right)\right) \\
& +a_{2} \cos \theta_{i} \cos \theta_{n}+a_{2} \sin \theta_{i} \sin \theta_{n} \cos \left(\phi_{i}-\phi_{n}\right) \\
= & a_{1} \exp \left(-2 c^{2}+2 c^{2} \cos \theta_{i} / 2 \cos \theta_{n}\right) g\left(\cos \left(\phi_{i}-\phi_{n}\right)\right) \\
& +a_{2} \cos \theta_{i} \cos \theta_{n}+a_{2} \sin \theta_{i} \sin \theta_{n} \cos \left(\phi_{i}-\phi_{n}\right)
\end{aligned}
$$

where, we have set $\alpha=2 c^{2} \sin \theta_{i} / 2 \sin \theta_{n}$. Consider the case when $\phi_{n}=0$. When viewed as a function of $\phi_{i}, I()$ is simply the sum of $g()$, a d.c. term and a $1^{\text {st }}$ harmonic term. The low pass nature is preserved and we can sample $I\left(\phi_{i}\right)$ at the same rate as we sampled $g\left(\cos \phi_{i}\right)$ to retain all the information in it. Sampling $I\left(\phi_{i}\right)$ consists of uniformly placing as many light sources as we need in one period at any constant zenith angle. The intensities at any pixel obtained by imaging the surface with one light source at a time gives the sample values of $I\left(\phi_{i}\right)$ for that pixel. Further, note that when $\phi_{n}$ is zero, $I\left(\phi_{i}\right)$ is an even function of $\phi_{n}$. The phases of all the DFT terms of $I\left(\phi_{i}\right)$ are zero. A non-zero $\phi_{n}$ corresponds to a circular shift of $I\left(\phi_{i}\right)$ (see equation (6.2)). From standard DFT theory we know that the phase of the non-dc terms will be different in this case. $\phi_{n}$ can be estimated by

$$
\phi_{n}=\tan ^{-1} \frac{\operatorname{Im} \operatorname{DFT}\left(I\left(\phi_{i}\right), 1\right)}{\operatorname{Real} \operatorname{DFT}\left(I\left(\phi_{i}\right), 1\right)}
$$

where, if $N$ is the number of samples

$$
\operatorname{DFT}\left(I\left(\phi_{i}\right), k\right)=\sum_{i} I\left(\phi_{i}\right) e^{-j 2 \pi k i / N} .
$$

Thus, we can estimate $\phi_{n}$ in a non-parametric manner independent of the rest of the parameters. The number of light sources needed to obtain the solution to $\phi_{n}$ is given by the Nyquist sampling rate for $g^{*}(\phi)$.

Central to this whole idea is the fact that $g\left(\cos \phi_{i}\right)$ can be approximated as a bandlimited function. The harmonic at which its spectrum begins to drop off is given by $k^{*}$, which by the above analysis is

$$
\begin{aligned}
k^{*} & =[1 / 2(\alpha-1)] \\
& =\left[1 / 2\left(2 c^{2} \sin \theta_{i} / 2 \sin \theta_{n}-1\right)\right] \\
& \leq\left[1 / 2\left(2 c^{2} \sin \theta_{i} / 2-1\right)\right],
\end{aligned}
$$




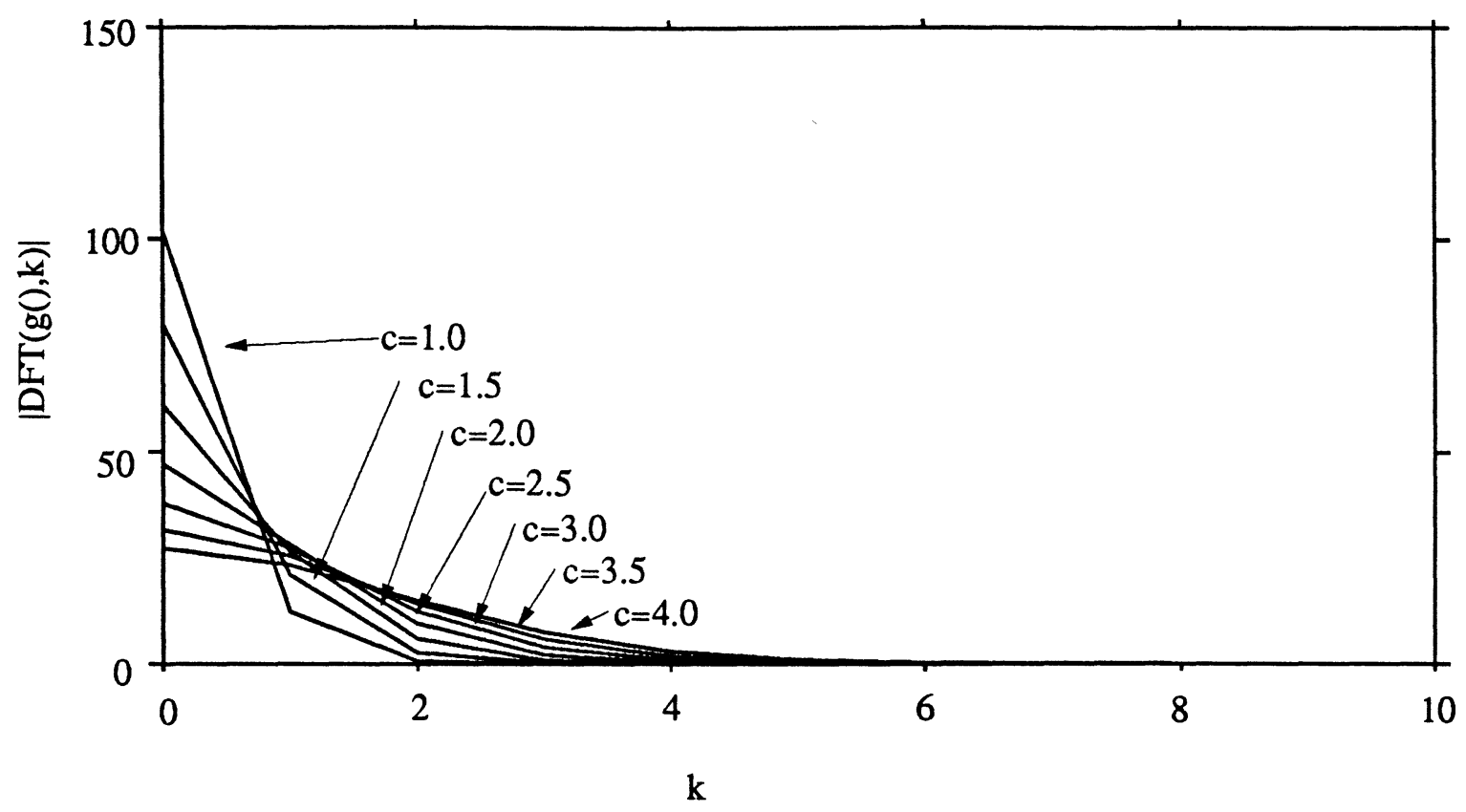

Figure 6.1 Amplitude spectrum of $g(\cos \phi)$ for different $c$

where, $[x]$ is the smallest integer greater than or equal to $x$. Thus, $k^{*}$ can be approximately evaluated by setting it to $\left[1 / 2\left(2 c^{2} \sin \theta_{i} / 2-1\right)\right]$. For $c=2.578$ and $\theta_{i}=40.0^{\circ}$, this yields $k^{*}=2$. Hence, we expect the spectrum to fall off after the third harmonic. However, the spectrum falls off much faster than this bound suggests. Figure 6.1 shows the amplitude spectrum of $g\left(\cos \phi_{i}\right)$ with $\theta_{i}=40^{\circ}, \theta_{n}=20^{\circ}$ for different values of $c$. Note the sharp cutoff of the amplitude spectrum. In particular note that for $c=2.578$ the function $g\left(\cos \phi_{i}\right)$ is band limited to the fourth harmonic.

If $c$ is large and the lobe width small, extended light sources can be used to decrease the bandwidth of $I\left(\phi_{i}\right)$ [39]. Since the reflectance map that we are using for experimental purposes has $c=2.578$, from figure 6.1 we note that the bandwidth of this function is essentially $k=4$ and we can use eight or more light sources in order to obtain $\phi_{n}$ from equation (6.4).

Having estimated $\phi_{n}$ let us turn to the rest of the parameters. Let us rotate our reference frame such that in the new frame we have $\phi_{n}=0$ and the same $\theta_{n}$ as before. Let us use the approximation for $\cos ^{-1}$ and write the reflectance map as

$$
I(\mathbf{i}, \mathbf{n}, \mathbf{r})=a_{1} \exp \left(-c \cos ^{-1} \mathbf{n}_{\mathbf{s}}^{T} \mathbf{n}\right)^{2}+a_{2} \mathbf{i}^{T} \mathbf{n}
$$




$$
\begin{aligned}
\cong & a_{1} \exp -c^{2}\left(2-\mathbf{n}_{\mathbf{s}}{ }^{T} \mathbf{n}\right)+a_{2} \mathbf{i}^{T} \mathbf{n} \\
= & a_{1} \exp \left(-2 c^{2}\right) \exp \left(2 c^{2} \cos \theta_{i} / 2 \cos \theta_{n}+\sin \theta_{i} / 2 \cos \theta_{n} \cos \phi_{i}\right) \\
& +a_{2}\left(\cos \theta_{i} \cos \theta_{n}+\sin \theta_{i} \sin \theta_{n} \phi_{i}\right) \\
= & a_{1} \exp \left(-2 c^{2}+2 c^{2} \cos \theta_{i} / 2 \cos \theta_{n}\right) \exp \left(2 c^{2} \sin \theta_{i} / 2 \cos \theta_{n} \cos \phi_{i}\right) \\
& +a_{2} \sin \theta_{i} \sin \theta_{n} \cos \phi_{i}+a_{2} \cos \theta_{i} \cos \theta_{n} \\
= & a_{1}^{*} \exp \left(c^{*} x_{i}\right)+a_{2}^{*} x_{i}+a_{3}^{*}
\end{aligned}
$$

where, $a_{1}^{*}=a_{1} \exp \left(-2 c^{2}+c^{2} \cos \theta_{i} / 2 \cos \theta_{n}\right), a_{2}^{*}=a_{2} \sin \theta_{i} \sin \theta_{n}, a_{3}^{*}=a_{2} \cos \theta_{i} \sin \theta_{n}$, $c^{*}=2 c^{2} \sin \theta_{i} / 2 \cos \theta_{n}$, and $x_{i}=\cos \phi_{i}$. Note that we know the $x_{i}$ a priori and have $I_{i}$ as measurements.

We ask: how many different $x_{i}$ do we need so that the set of equations given by (6.5) can be uniquely solved for $a_{1}^{*}, a_{2}^{*}$, and $a_{3}^{*}$ ?

Lemma 6.1 Four distinct values of $x_{i}$ are necessary to solve equation (6.5) for $a_{1}^{*}, a_{2}^{*}$ and $a_{3}^{*}$ in a globally unique fashion.

Proof To begin, note that the equation

$$
\alpha_{1} \exp \left(c_{1} x\right)-\alpha_{2} \exp \left(c_{2} x\right)=\beta_{1} x+\beta_{2}
$$

can have at most three solutions for $x$ given any values of $\alpha_{1}, \alpha_{2}, \beta_{1}, \beta_{2}$ and $c_{1}, c_{2}$. This is seen as follows: Let us denote the left hand side of equation (6.6) by $f(x)$ and rewrite it as

$$
f(x)-\beta_{1} x+\beta_{2}=0 .
$$

If $x_{1}, x_{2}, \cdots, x_{n}$ are the solutions of equation (6.6) placed in ascending order, then, between any two consecutive solutions, the derivative of the left hand side of equation (6.7) goes to zero at least once, i.e., if the number of solutions to

$$
f^{\prime}(x)-\beta_{1}=0
$$

is $m$, then $n \leq m+1$. Using the same argument on equation (6.8) we have that if

$$
f^{\prime \prime}(x)=0
$$

has $k$ solutions, then $m \leq k+1$. Hence, $n \leq k+2$. But equation (6.9) can be written as $\alpha_{1} c_{1}^{2} \exp \left(c_{1} x\right)=\alpha_{2} c_{2}^{2} \exp \left(c_{2} x\right)$. Taking logarithms we get, $c_{1} x=c_{2} x+\log \alpha_{2} c_{2}^{2}-$ 
$\log \alpha_{1} c_{1}^{2}$ which is a linear equation and has a single solution. Hence $k=1$ and $n=2$. Thus equation (6.6) has at most 3 roots. (It is easy to find $\alpha_{1}, \alpha_{2}, c_{1}, c_{2}, \beta_{1}, \beta_{2}$ that cause the equation (6.6) to actually have three roots.)

We will now prove the lemma with a contradiction. Suppose that for four different values of $x_{i}$ we can find two different sets $\left(a_{1}^{*}, a_{2}^{*}, a_{3}^{*}, c^{*}\right)$ and $\left(\tilde{a}_{1}^{*}, \tilde{a}_{2}^{*}, \tilde{a}_{3}^{*}, \tilde{c}^{*}\right)$ such that for the values of $x_{i}$, we obtain identical values of $I\left(x_{i}\right)$. Then, from equation (6.5) $\forall i$

$$
a_{1}^{*} \exp \left(c_{1}^{*} x_{i}\right)+a_{2}^{*} x_{i}+a_{3}^{*}=\tilde{a_{1}^{*}} \exp \left(\tilde{c_{1}^{*}} x_{i}\right)+\tilde{a_{2}^{*}} x_{i}+\tilde{a_{3}^{*}}
$$

i.e.,

$$
a_{1}^{*} \exp \left(c_{1}^{*} x_{i}\right)-\tilde{a}_{1}^{*} \exp \left(\tilde{c}^{*} x_{i}\right)=\left(\tilde{a_{2}^{*}}-a_{2}^{*}\right) x_{i}+\tilde{a}_{3}^{*}-a_{3}^{*}, \quad i=1, \cdots, 4
$$

This has the same form as equation (6.6) for which we know there cannot be more that three solutions. Hence, we have a contradiction.

Before we proceed, note that we need four distinct values of $x_{i}$. This does not mean that we can use four lights. The symmetry in the light placement that we are using forces us to use twice as many light sources. This situation is shown in figure 6.2. Note that if $\phi_{n}=\phi_{i_{1}}$ (or any other $\phi_{i}$ ) we get only 4 different $\cos \phi_{i}$ for eight light sources.

We conclude that the number of light sources that are sufficient to obtain a globally unique solution to equation (6.1) is greater than or equal to eight. We will need more than eight light sources if the bandwidth of $I\left(\phi_{i}\right)$ is large and $\phi_{n}$ cannot be estimated via equation (6.4) with eight lights. Note that for the reflectance map of (3.19) with $c=2.578$ the bandwidth is limited in such a fashion that eight light sources are sufficient to provide Nyquist sampling. Hence, eight light sources are sufficient to obtain a solution for $\phi_{n}$ as well as $\theta_{n}, a_{1}$ and $a_{2}$.

\subsection{Ill-posed Nature of the Joint Estimate}

The considerations of the previous section give a theoretical estimate of the number of light sources necessary to solve (6.1) in a globally unique fashion. The answer rests on the property that equation (6.6) has a limited number of solutions. However, if the data is noisy, we can rewrite equation (6.6) as $\alpha_{1} \exp \left(c_{1} x\right)-\alpha_{2} \exp \left(c_{2} x\right)-\beta_{1} x-\beta_{2}=$ $n(x)$, where $n(x)$ is a white, Gaussian noise process. The number of solutions of this equation are now given by the number of crossings by the noise process to the 


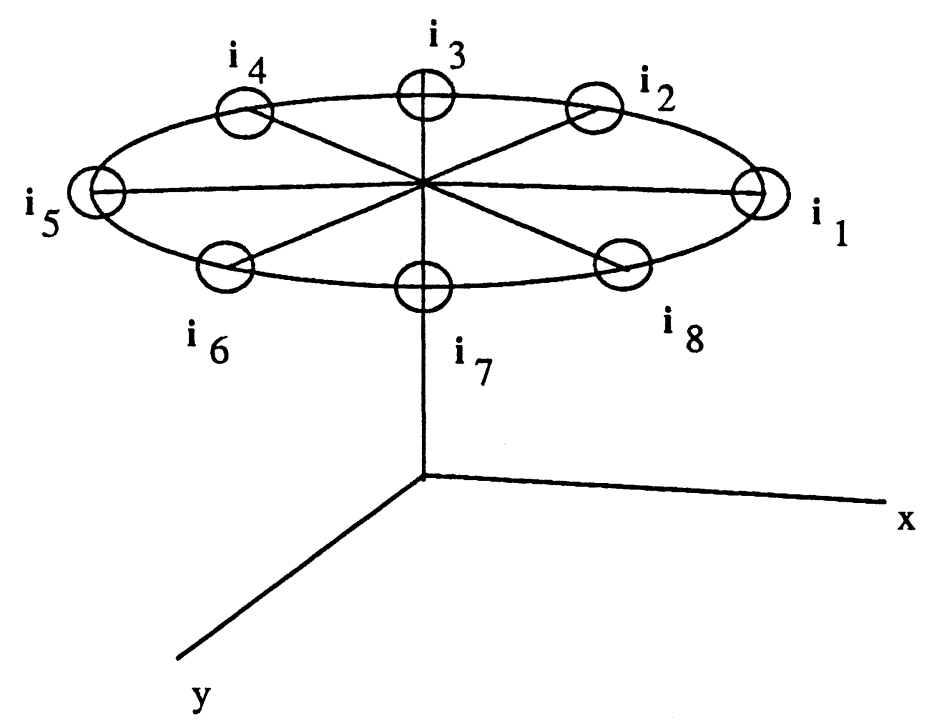

Figure 6.2 Eight lights for 4 different $x_{i}$.

curve $\alpha_{1} \exp \left(c_{1} x\right)-\alpha_{2} \exp \left(c_{2} x\right)-\beta_{1} x-\beta_{2}$. It is well known [16] that this can be an arbitrarily large number even for small amount of noise. Thus, we are faced with the possibility that the problem of obtaining a unique solution to equation (6.6) may be ill-posed [51] even if the number of light sources is large.

In this section, we will see that this is indeed so. Since equation (6.1) is highly non-linear it is difficult to demonstrate this rigorously. We will demonstrate the illposed nature in two ways. First, we will set up an equivalent linear problem and show that the linear problem is ill-posed. Besides establishing ground for belief in the ill-posedness of equation (6.1) the linear analysis also gives us qualitative insight into the origin of the ill-posed nature of the problem. Second, we will numerically compute the right hand side of equation 6.1 and show that large changes in $\mathbf{n}$ cause small changes to the intensity vector.

\section{Linear Analysis}

The function $\exp \left(-c \cos ^{-1}()\right)^{2}$ can be approximated quite well as a linear function over a large range of its domain. Figure 6.3 shows the function for $c=2.578$ and two linear fits over different ranges. Let us assume that for a particular surface normal $\mathbf{n}$ and a set of light sources, the sequence of numbers $\mathbf{n}_{\mathbf{s}_{\mathbf{i}}}{ }^{T} \mathbf{n}$ lie in one of these regions. Then, we can approximate the function $\phi(x)=\exp \left[-c^{2}\left\{\cos ^{-1}(x)\right\}^{2}\right]$ 


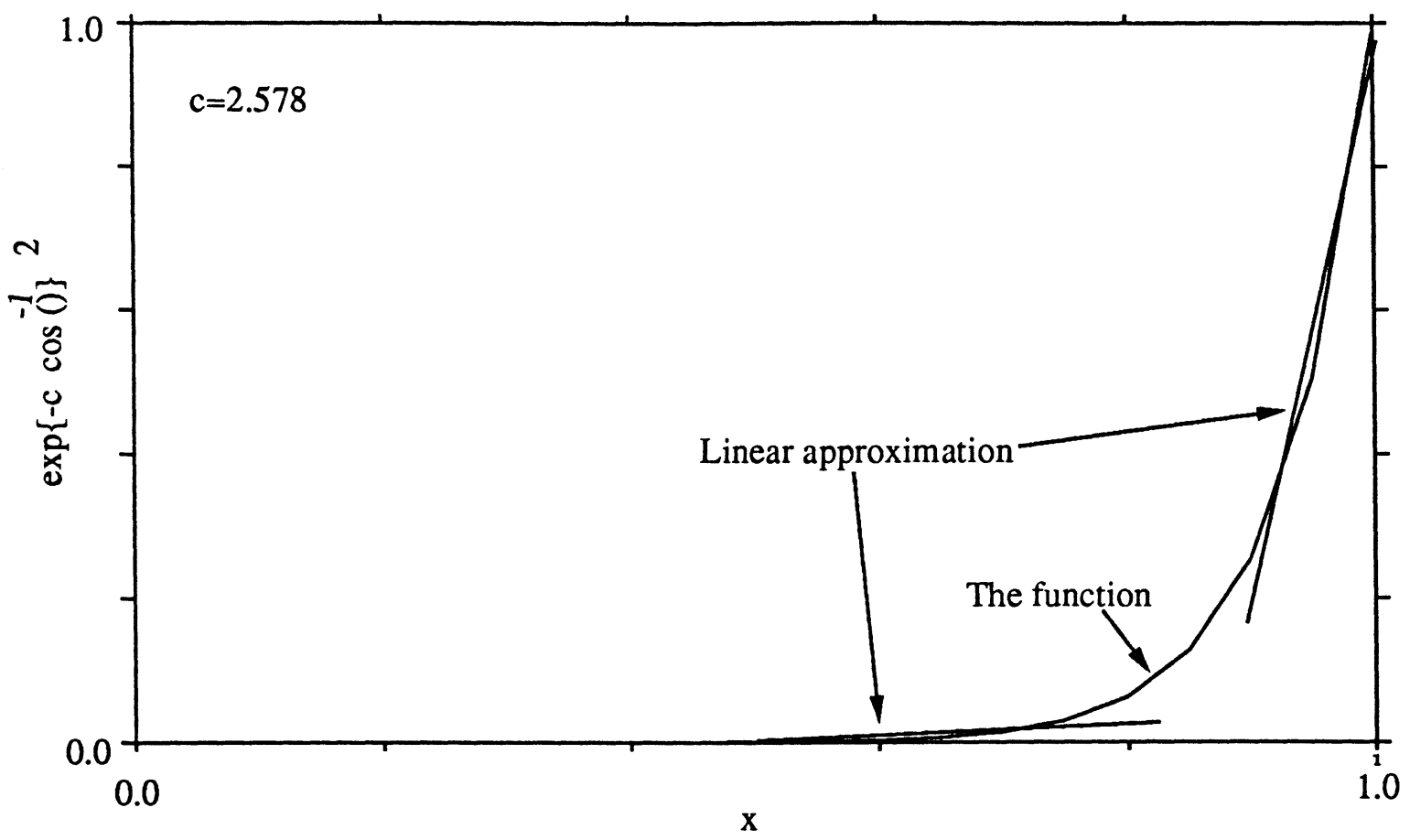

Figure 6.3 Linear approximation to the forescatter lobe function.

as $\phi(x) \cong \phi\left(x_{0}\right)+\phi^{\prime}\left(x_{0}\right)\left(x-x_{0}\right)$, where $x_{0}$ is some number in the domain of $\phi()$ that we are approximating within. Equation (6.1) now reduces to:

$$
\begin{aligned}
\left(\begin{array}{c}
I\left(\mathbf{i}_{1}, \mathbf{n}, \mathbf{r}\right) \\
\vdots \\
I\left(\mathbf{i}_{k}, \mathbf{n}, \mathbf{r}\right)
\end{array}\right)= & a_{1}\left(\phi\left(x_{0}\right)-x_{o} \phi^{\prime}\left(x_{0}\right)\right)\left(\begin{array}{c}
1 \\
\vdots \\
1
\end{array}\right) \\
& +a_{1} \phi^{\prime}\left(x_{o}\right)\left(\begin{array}{c}
\mathbf{n}_{\mathbf{s}_{1}^{T}}^{T} \\
\vdots \\
\mathbf{n}_{\mathbf{s}_{k}^{T}}^{T}
\end{array}\right) \mathbf{n} \\
& +a_{2}\left(\begin{array}{c}
\mathbf{i}_{1}^{T} \\
\vdots \\
\mathbf{i}_{k}^{T}
\end{array}\right) \mathbf{n} \\
= & P u .
\end{aligned}
$$


where,

$$
u=\left(\begin{array}{c}
a_{1} \phi^{\prime}\left(x_{o}\right) \mathbf{n} \\
a_{2} \mathbf{n} \\
a_{1}\left\{\phi\left(x_{o}\right)-x_{o} \phi^{\prime}\left(x_{o}\right)\right\}
\end{array}\right)
$$

and,

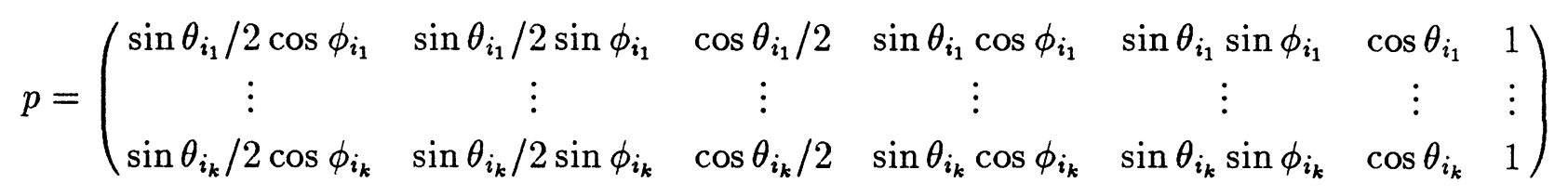

Note that the vector $u$ has vertical dimension of 7.( Each $\mathbf{n}$ has a dimension of 3.)

Under the assumptions of chapter 3 that $\theta_{i_{k}} \leq 30^{\circ}$, we have the following

1. $\cos \theta_{i_{k}} / 2$ and $\cos \theta_{i_{k}}$ are close to 1 . Thus columns 3,6 and 7 in the matrix above are close to being linearly dependent.

2. $\sin \theta_{i_{k}} / 2 \cong 1 / 2 \sin \theta_{i_{k}}$. Hence the pairs of columns 1,4 and 2,5 are close to being linearly dependent.

Thus the matrix $P$ has three linearly independent columns and hence only three quantities in the vector $u$ can be estimated with any accuracy at all. Since, our ultimate goal is to estimate four quantities $\left(a_{1}, a_{2}, \theta_{n}, \phi_{n}\right)$, the problem becomes ill-posed. Note that the ill-posed nature of the problem is independent of the vertical dimension of $P$ and is hence independent of the number of light sources.

Qualitatively speaking, we state that the ill-posed nature comes about by

1. Remark [1] above implies that the part of observed intensity vector that lies along $\left[\begin{array}{lll}1 & 1 & \cdots\end{array}\right]^{T}$ cannot be easily decomposed into contributions due to the forescatter and normal lobe, i.e., there is no way of telling how much of the "constant" part of the intensity values was generated by either lobes.

2. Remark [2] implies that for the range of incidence angles we are interested in, the variation in intensity due to the change in the zenith angle $\theta_{i_{k}}$ also cannot be uniquely decomposed into contributions due to either lobes.

\section{Numerical Analysis}

The major assumption of the foregoing analysis has been that the values of $\mathbf{n}_{\mathbf{s}}{ }_{k}^{T} \mathbf{n}$ we obtain for the light placements fall in a region such that $\phi\left(\mathbf{n}_{\mathbf{s}}{ }_{k}^{T} \mathbf{n}\right)$ is effectively a 
linear function. If the lobe width is narrow and $\phi()$ is not significantly linear over any reasonable part of its domain, then this non-linearity can enhance the differences in the columns of $P$, curing the problem of its ill-posedness. However, if the lobe-width is significant, the problem can become ill-posed.

For the exact non-linear equation (6.2) the ill-posed nature is shown in figure 6.4. The figure shows a plot of $\left\|I_{1}\left(\theta_{i}, \phi_{i}\right)-I_{2}\left(\theta_{i}, \phi_{i}\right)\right\|$ for $0 \leq \theta_{i} \leq 30^{\circ}$ and $0 \leq \phi_{i}<360^{\circ}$. The functions $I_{1}()$ and $I_{2}()$ are specific cases of equation (6.2) where the following parameters were used. For $I_{1}(), a_{1}=140.0, a_{2}=70.0, c=2.578, \theta_{n}=33.0^{\circ}, \phi_{n}=$ $0.0^{\circ}$, and for $I_{2}(), a_{1}=98.0, a_{2}=0.0, c=2.578, \theta_{n}=10.32^{\circ}, \phi_{n}=0.0$. Thus, $I_{1}()$ corresponds to a surface normal with an azimuth angle of zero degrees and a zenith angle of $33.0^{\circ}$ while $I_{2}()$ is the function for a surface normal with zenith angle of $10.32^{\circ}$ and the same azimuth angle. Note that the values of $a_{1}, a_{2}$ are different for both the cases. The range of values we have used correspond to the actual grey level values recorded by the camera in our experimental set up. In figure 6.4 , note that while the maximum difference between $I_{1}()$ and $I_{2}()$ is about 40 grey levels, the mean difference is only 6.1 grey levels. Also, the maximum difference occurs at the $\theta_{i}=0.0^{\circ}$, a value which is unlikely to occur in experiments. For non-zero $\theta_{i}$, the difference falls off of quite rapidly. The mean difference of 6.1 grey levels is well within the range of noise generated by most cameras. Hence, we conclude that it will be difficult to distinguish between the two surface normals and the two "albedos" even by using a large number of light sources in the region $0 \leq \phi_{i}<360^{\circ}$ and $0 \leq \theta_{i} \leq 30^{\circ}$. The problem is clearly ill-posed.

\subsection{Regularization of Joint Estimation}

Ill-posed problems can be regularized using Tikhonov's method [51]. The regularization consists of converting the algebraic problem of equation (6.1) into an optimization problem. Regularization terms are then added to the function being optimized. We will regularize equation (6.1) by saying that our estimates of $\mathrm{n}, a_{1}$ and $a_{2}$ are those that minimize $Q$,

$$
Q=\lambda_{1}(I-\tilde{I})^{T}(I-\tilde{I})+\lambda_{2}\left(a_{1}-\alpha_{1}\right)^{2}+\lambda_{3}\left(a_{2}-\alpha_{2}\right)^{2},
$$

where,

$$
I=\text { vector of observed intensities }
$$




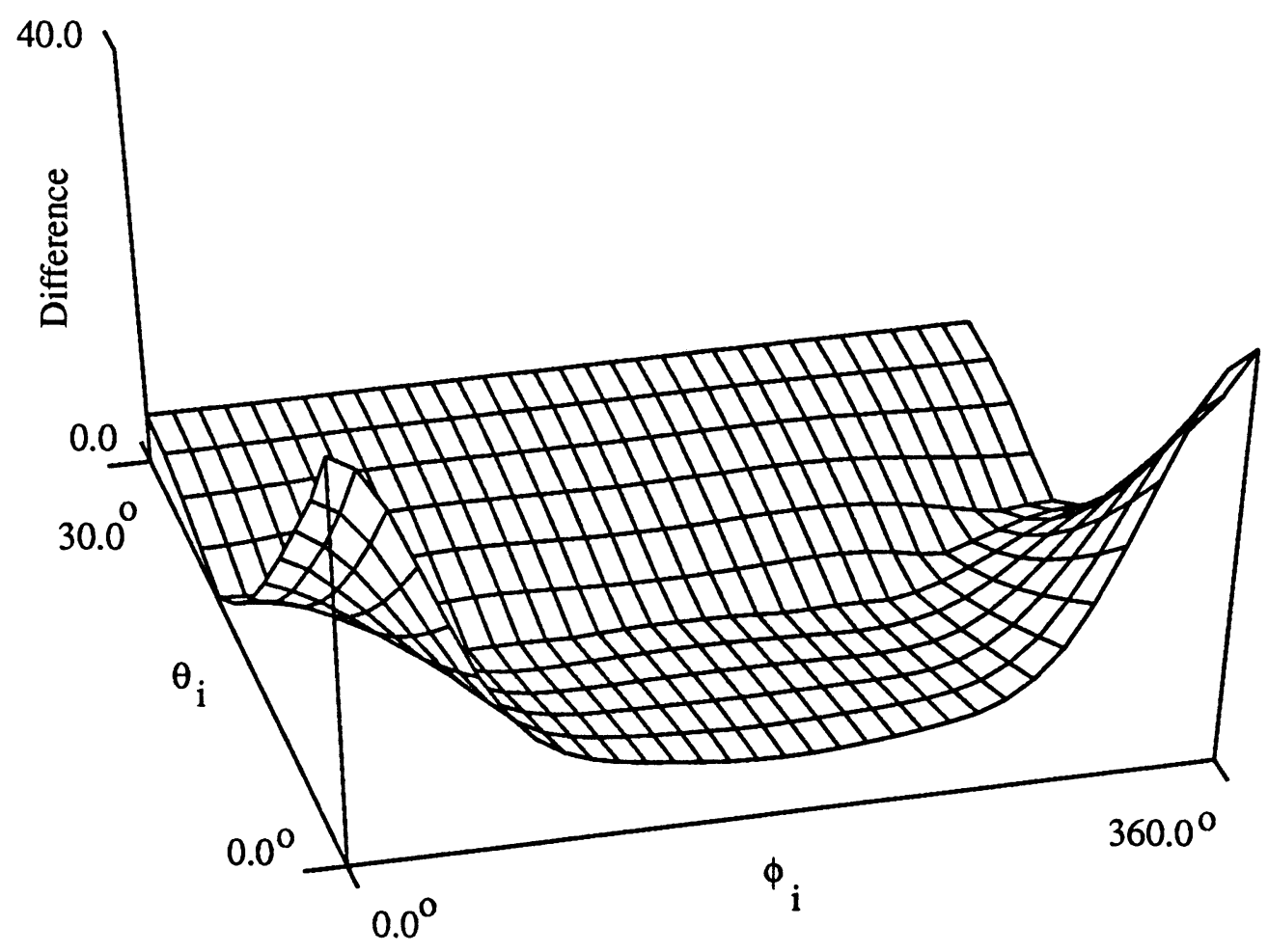

Figure 6.4 Ill-posed nature of the joint estimate. 


$$
\tilde{I}=a_{1}\left(\begin{array}{cc}
\exp (-c & \left.\cos ^{-1} \mathbf{n}_{\mathbf{s}}^{T} \mathbf{n}\right)^{2} \\
\vdots & \\
\exp (-c & \left.\cos ^{-1} \mathbf{n}_{\mathbf{s} k}^{T} \mathbf{n}\right)^{2}
\end{array}\right)+\mu a_{2}^{*}\left(\begin{array}{c}
\mathbf{i}_{1}^{T} \mathbf{n} \\
\vdots \\
\mathbf{i}_{k}^{T} \mathbf{n}
\end{array}\right)
$$

$\lambda_{1}, \lambda_{2}, \lambda_{3}>0$ and $\alpha_{1}$ and $\alpha_{2}$ are some a priori estimates of $a_{1}$ and $a_{2}$. This is a non-linear optimization problem in $\mathrm{n}, a_{1}$ and $a_{2}$ and a closed form solution cannot be obtained for it. We will use a standard non-linear, numerical optimization procedure [30] to achieve this.

The entire process of joint estimation for surfaces with reflectance map given by equation (6.2) and $c=2.578$ is

1. The surface is imaged with eight light sources placed at azimuth angles of $0^{\circ}, \pm 45^{\circ}, \pm 90^{\circ}, \pm 135^{\circ}, 180^{\circ}$ and at a constant zenith angle of $25^{\circ}$.

2. We know from section 6.2 that these light sources are sufficient to obtain an estimate of $\phi_{n}$. The estimate is obtained via equation (6.4).

3. The other parameters are obtained by solving the regularized problem of equation (6.10). The exact function used in doing this was a slight modification of $Q$ as defined in equation (6.10). The following function was minimized

$$
Q=\lambda_{1}\left(I-I^{*}\right)^{T}\left(I-I^{*}\right)+\lambda_{2}\left(a_{1}^{*}-\alpha_{1}^{*}\right)^{2}+\lambda_{3}\left(a_{2}^{*}-\alpha_{2}^{*}\right)^{2}
$$

where,

$$
I^{*}=\mu a_{1}^{*}\left(\begin{array}{cc}
\exp (-c & \left.\cos ^{-1} \mathbf{n}_{\mathbf{s}}^{T} \mathbf{n}\right)^{2} \\
\vdots & \\
\exp (-c & \left.\cos ^{-1} \mathbf{n}_{\mathbf{8} k}^{T} \mathbf{n}\right)^{2}
\end{array}\right)+\mu a_{2}^{*}\left(\begin{array}{c}
\mathbf{i}_{1}^{T} \mathbf{n} \\
\vdots \\
\mathbf{i}_{k}^{T} \mathbf{n}
\end{array}\right)
$$

where, $\mu$ is a scaling factor explained below. The optimization was done using two constraints

$$
\mathbf{n}^{T} \mathrm{n}=1 \quad \text { and } \quad \mathbf{v}^{T} \mathbf{n}=0
$$

where, $\mathbf{v}=\left[\begin{array}{lll}-\sin \phi_{n} & \cos \phi_{n} & 0\end{array}\right]^{T}$. The constraint $\mathbf{v}^{T} \mathbf{n}=0$ forces the azimuth angle of $\mathbf{n}$ to be $\phi_{n}$. As mentioned before the IMSL package [30] was used to obtain the optimization. 


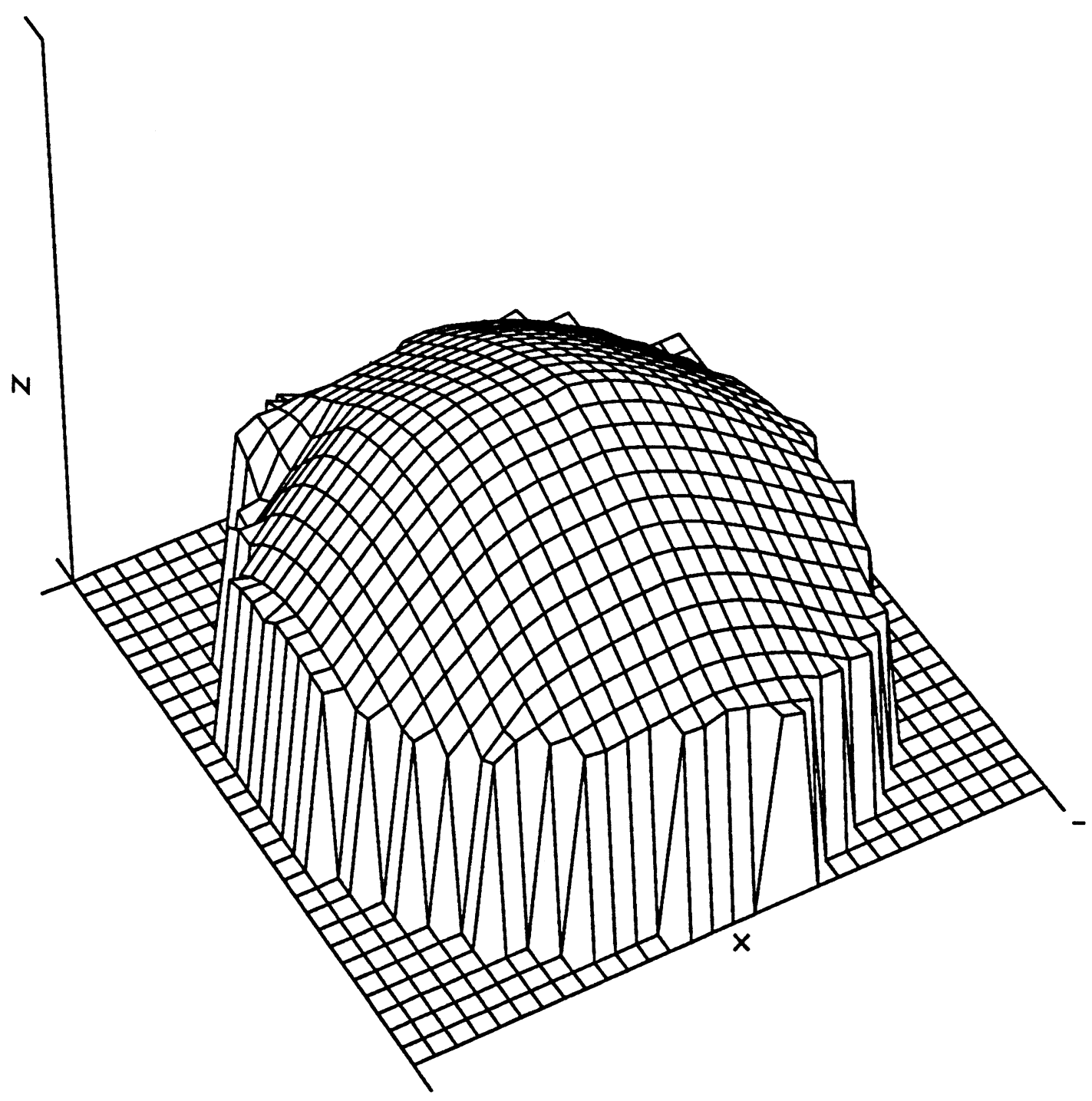

Figure 6.5 Regularized reconstruction of the sphere. 


\subsection{Experimental Results}

Figure 6.5 shows the reconstruction of the spherical surface which had the reflectance map of equation (3.22). It was easily determined that for the illumination conditions of this experiment, the reflectance map for the surface would be best described by $\mu=140.0, \alpha_{1}^{*}=1.0$ and $\alpha_{2}^{*}=0.5$. The value of $\mu=140.0$ was used in equation (6.12) while $\alpha_{1}^{*}$ and $\alpha_{2}^{*}$, the a priori guesses in equation (6.11) were set to 1.1 and 0.45 respectively. Thus, we did not assume exact knowledge of the albedos.

The constants in the minimization function of (6.11) were $\lambda_{1}=100, \lambda_{2}=1600$, and $\lambda_{3}=900$. As we shall see, this is not an excessive amount of regularization. The initial values for the optimization procedure were $\mathbf{n}=\left[\begin{array}{lll}0 & 0 & 1\end{array}\right]^{T}$ and $a_{1}^{*}=1.2$ and $a_{2}^{*}=0.8$.

The mean square deviation of the reconstructed surface normals from the best fit spherical surface was calculated as before and a value of $\Delta \theta_{\text {error }}=5.21^{\circ}$ was obtained. From the results of section 4.5 note that this is worse than the un-normalized reconstruction results but not as bad as the results obtained by using the Lambertian model.

Figures 6.6 and 6.7 show the histograms of the values of $a_{1}^{*}$ and $a_{2}^{*}$ as returned by the optimization procedure for the entire surface. The peaks in the distribution occur for $a_{1}$ at 1.04 and $a_{2}$ at 0.34 . From the distribution, note that spread of the values is fairly large and this corresponds to a mild amount of regularization. Finally, figures 6.8 and 6.9 show reconstructions for the cylinder and spoon of figures 4.10 and 4.11 . The exact same procedure as used in these reconstructions as for the spherical surface. 


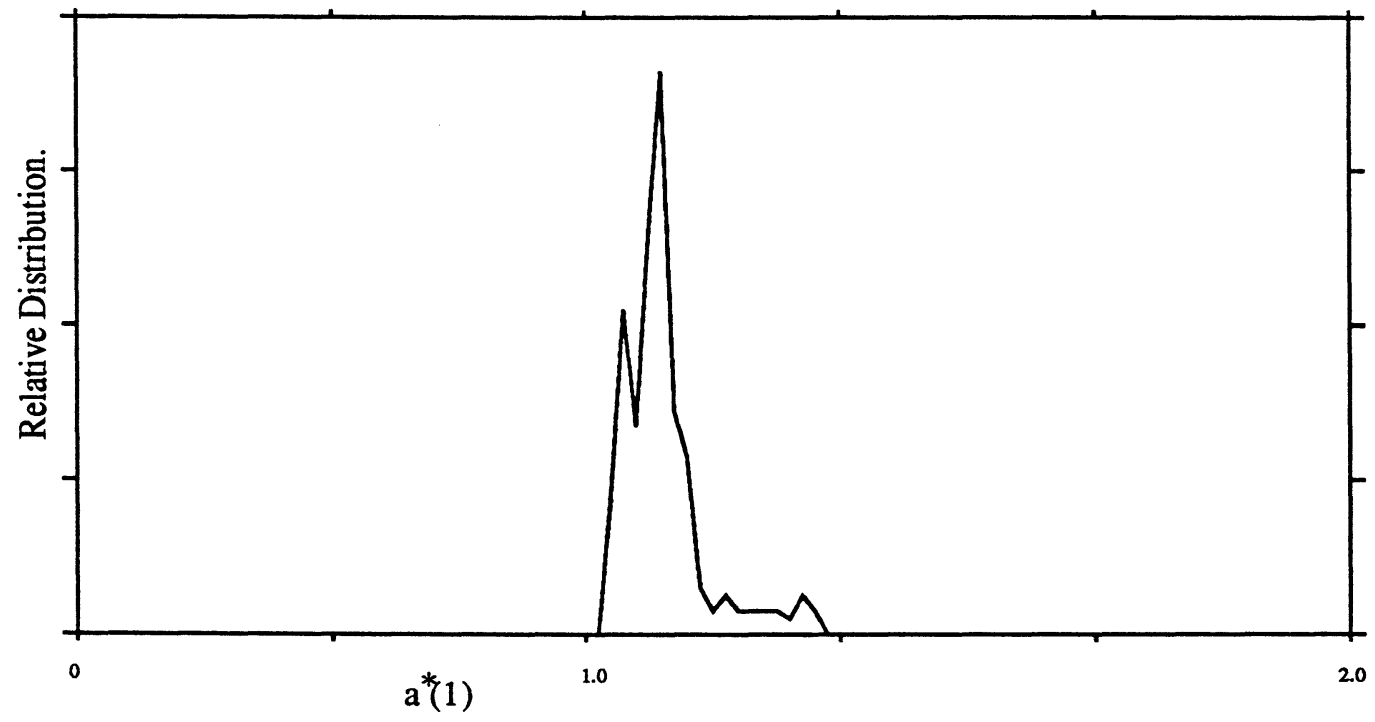

Figure 6.6 Histogram for $a_{1}^{*}$. 


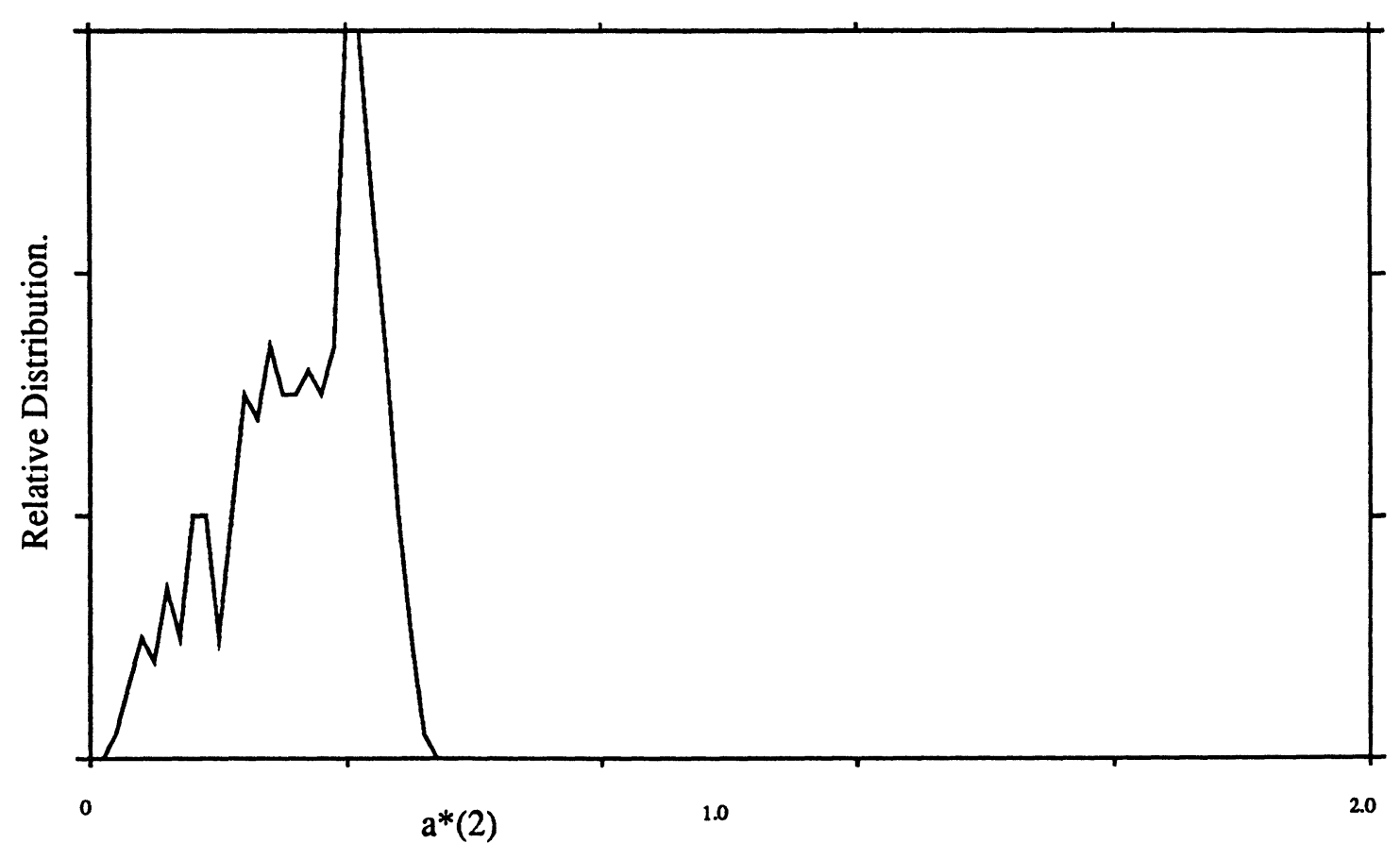

Figure 6.7 Histogram for $a_{2}^{*}$. 


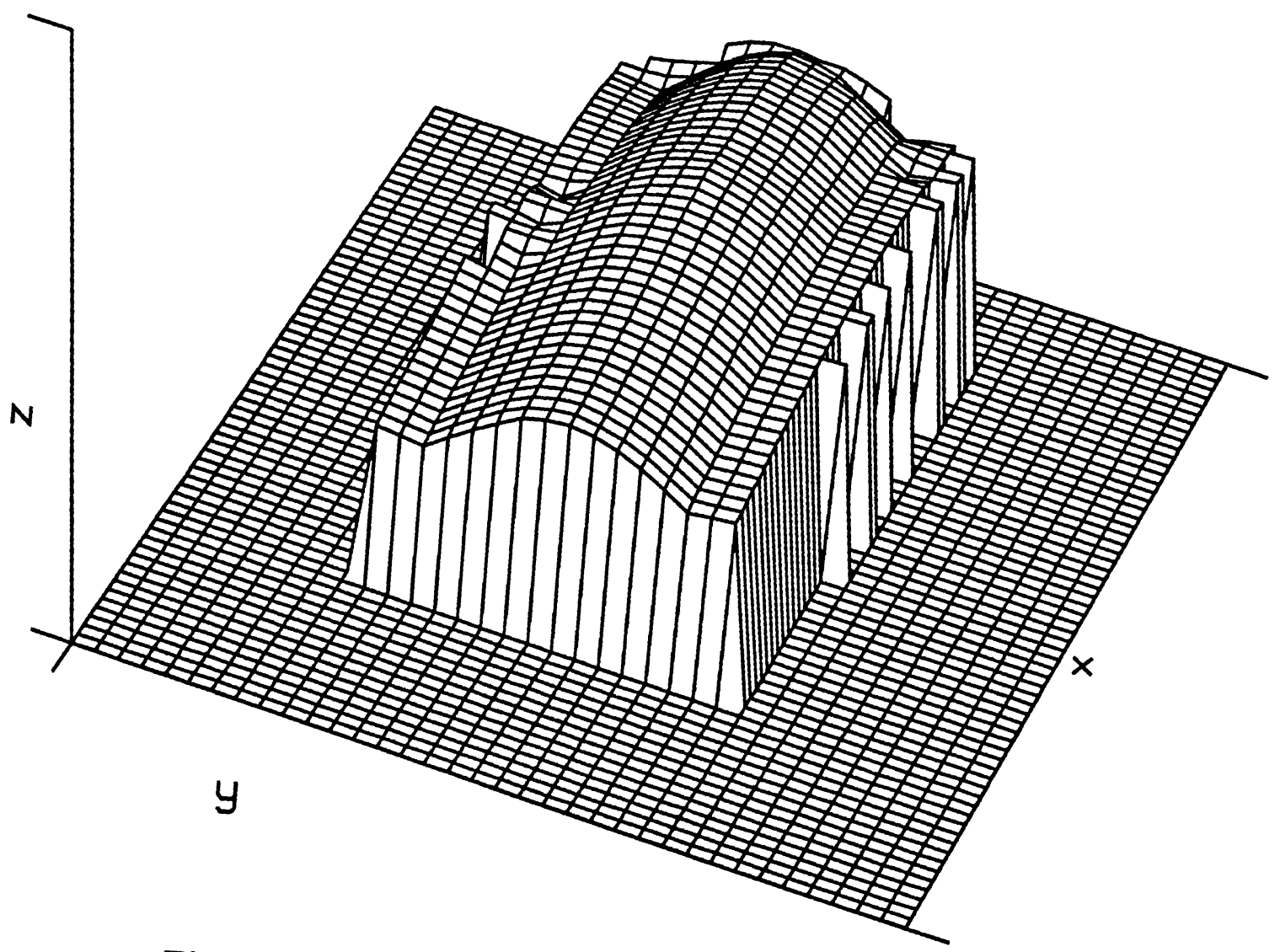

Figure 6.8 Regularized reconstruction of the cylinder. 


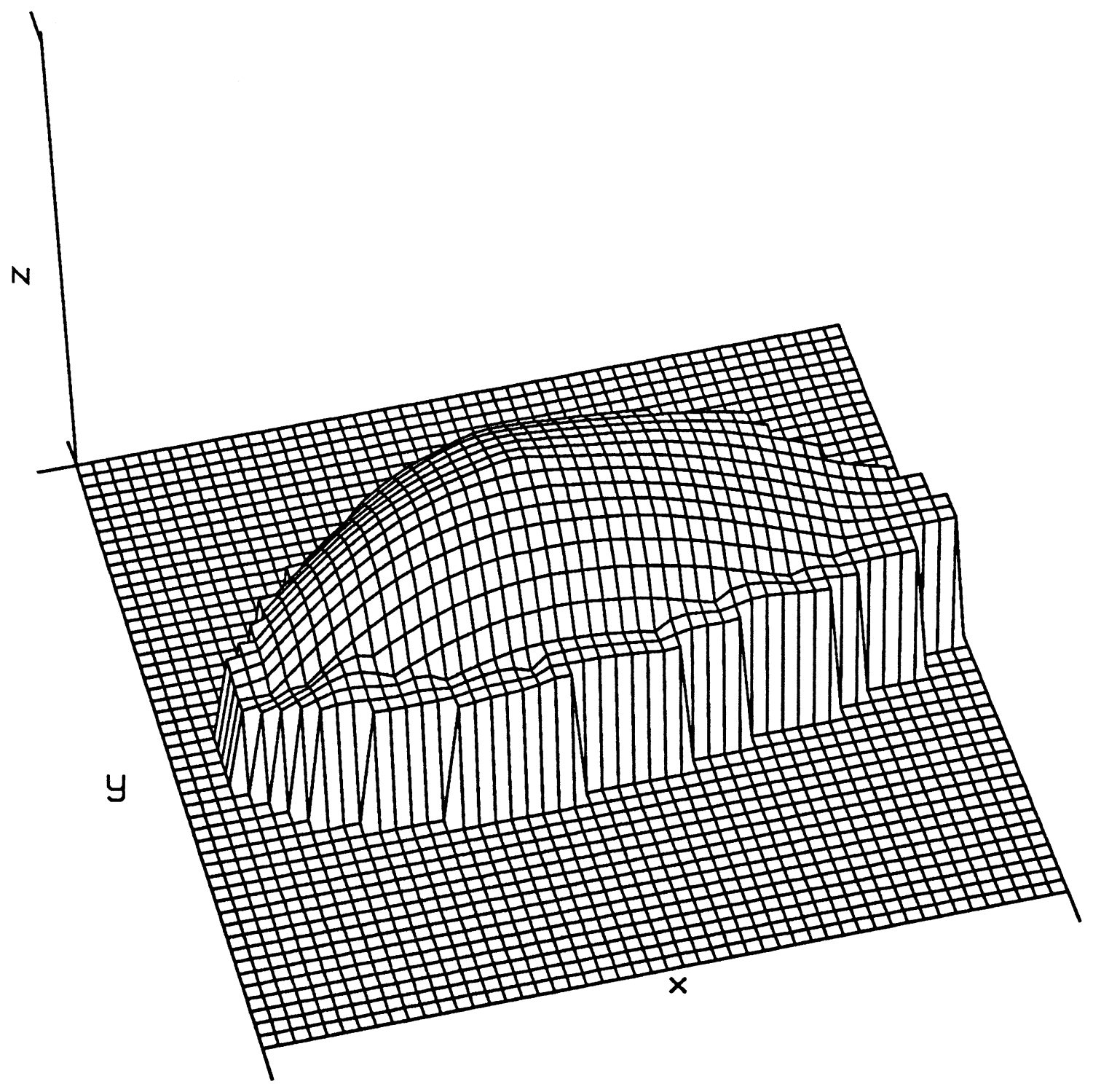

Figure 6.9 Regularized reconstruction of the spoon. 


\section{Chapter 7}

\section{Completeness of Photometric Inversion}

\subsection{Introduction}

In this chapter, we will consider the completeness problem. Suppose that for a given reflectance map we have determined that $k$ light sources are necessary for a globally unique solution of the surface normal and other parameters. If we use only $k$ light sources, then it is possible that parts of the surface will lie in shadow of one of the lights. These portions of the surface cannot be reconstructed. In order to completely reconstruct the surface, more than $k$ light sources may be needed so that at least $k$ of these illuminate every point on the object surface. We will call the problem of finding the exact number of light sources so that a given surface is illuminated at every point by a certain number of them, the completeness problem.

The completeness problem is best illustrated by the 2-dimensional example of figure 7.1. The figure shows a curve for which it has been a priori determined that two light sources are sufficient to obtain a globally unique solution for the surface normal. If only two light sources are used from the directions $\mathbf{i}_{\mathbf{1}}$ and $\mathbf{i}_{\mathbf{2}}$ as shown, note that the regions $S_{1}$ and $S_{2}$ lie in the shadow of one of the light sources and cannot be reconstructed. Since the camera is positioned along the vertical, we are constrained to position light sources in non-vertical directions. It is obvious that in this case we need at least four light sources - two on each side of the vertical - in order that every point on the curve be illuminated by at least two light sources.

The answer to the completeness problem is not independent of the shape of surface. If the surface is sufficiently contorted even a very large number light sources will not illuminate it completely. In this chapter, we will restrict ourselves to convex surfaces. 


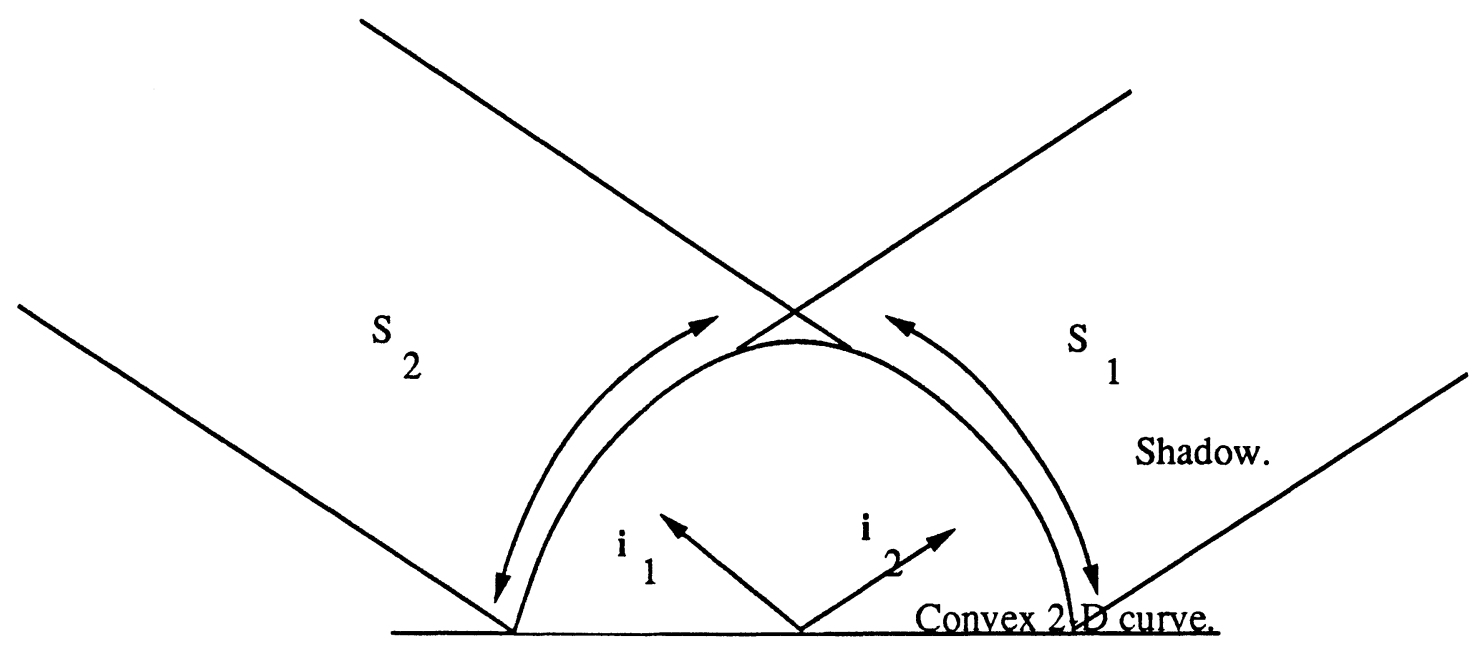

Figure 7.1 2 Light sources do not yield a complete reconstruction.

\subsection{Convex Surfaces}

If the surface is convex and a surface normal is not illuminated by a light source, then it lies in self-shadow with respect to that light source. The self-shadowing condition provides us the way to obtain an answer for the completeness problem. The terminology we will use is as follows. A vector $\mathbf{i}$ "illuminates" a vector $\mathbf{n}$ iff $\mathbf{i}^{T} \mathbf{n}>0$. A vector $\mathbf{n}$ is " $k$-illuminated" if there are $k i_{l}$ 's which illuminate n. The problem of completeness of photometric stereo can be stated as follows: How many vectors $\mathbf{i}_{l}, l=1,2, \ldots, k^{*}$ are required so that all vectors $\mathbf{n}$ are $\mathrm{k}$ illuminated $(k \geq 3)$ ? We are concerned with only those vectors $\mathbf{n}$ that have azimuth angles less than $\pi / 2$, i.e., the $\mathbf{z}$-component of $\mathbf{n}$ is strictly greater than zero. The light source direction vectors are constrained to be non-vertical, i.e., to have their zenith angles strictly greater than 0 .

As a first step in solving the completeness problem, we establish that constraining the position of light sources does not change $k^{*}$. This we do as follows: Given a vector $\mathbf{n}$, suppose that $\mathbf{i}_{l}, l=1, \cdots, k^{*}$ lights $\mathrm{k}$-illuminate $\mathbf{n}$. We now move the lights keeping their azimuth angles the same, but decreasing their zenith angles till they all have zenith angles equal to the smallest zenith angle of the 
initial configuration. Let us denote the new light sources by $\mathrm{i}_{l}^{\prime}, l=1, \cdots, k^{*}$. We will establish that if all of $i_{l}$ k-illuminate $n$, then so do all of $i_{l}^{\prime}$.

Lemma 7.1 If a vector $\mathbf{n}$ is illuminated by a given light source $\mathbf{i}$, then it is also illuminated by the light source $i^{\prime}$ which is given by

$$
\mathbf{i}^{\prime}=\left(\begin{array}{c}
\cos \phi \sin \theta^{\prime} \\
\sin \phi \sin \theta^{\prime} \\
\cos \theta^{\prime}
\end{array}\right)
$$

where, $0<\theta^{\prime}<\theta$, and

$$
\mathrm{i}=\left(\begin{array}{c}
\cos \phi \sin \theta \\
\sin \phi \sin \theta \\
\cos \theta
\end{array}\right)
$$

Proof Let us define two vectors $\mathbf{u}$ and $\mathbf{z}$ as

$$
\mathbf{u}=\left(\begin{array}{c}
\cos \phi \\
\sin \phi \\
0
\end{array}\right) \quad \mathbf{z}=\left(\begin{array}{l}
0 \\
0 \\
1
\end{array}\right)
$$

Then, we have $\mathbf{i}^{\prime}=\sin \theta^{\prime} \mathbf{u}+\cos \theta^{\prime} \mathbf{z}$ and $\mathbf{i}=\sin \theta \mathbf{u}+\cos \theta \mathbf{z}$. If we assume that $\mathbf{i}^{T} \mathbf{n}>0$, then we have $\mathbf{i}^{T} \mathbf{n}=\sin \theta \mathbf{u}^{T} \mathbf{n}+\cos \theta \mathbf{z}^{T} \mathbf{n}>0$. Since we know that $\mathbf{z}^{T} \mathbf{n}>0$, we have from the above inequality the following relation,

$$
\frac{1}{\tan \theta}>-\frac{\mathbf{u}^{T} \mathbf{n}}{\mathbf{z}^{T} \mathbf{n}}
$$

But we know that $\theta^{\prime} \leq \theta$, hence $\frac{1}{\tan \theta^{\prime}} \geq \frac{1}{\tan \theta}>-\frac{\mathbf{u}^{T} \mathbf{n}}{\mathbf{z}^{T} \mathbf{n}}$, which leads us to $\mathrm{i}^{T}>0$. Thus $\mathbf{i}^{\prime}$ also illuminates $\mathbf{n}$.

It now follows that if all $i_{l}^{\prime}$ have zenith angles less than or equal to the minimum zenith angle of $i_{l}$, then, $i_{l}^{\prime}$ will $\mathrm{k}$-illuminate any vector which is k-illuminated by $i_{l}$. We will now consider solving the completeness problem in a simplified setting: we will assume that all the light sources $i_{l}$ have been moved so that they have the same zenith angle.

Let $k^{*} \geq 3$. Given any $k^{*}$ light sources with the same zenith angle, we can number them by going around them in a counter clock wise manner. We assume 
that this is in fact the numbering $i_{l}$. As $k^{*} \geq 3$, there is unique way of defining the difference in azimuth angles between any two consecutive light sources $\mathbf{i}_{q}$ and $\mathbf{i}_{q+1}$. This is done by measuring in an anti-clock wise manner the change in the azimuth angle from $\phi_{q}$ to $\phi_{q+1}$, i.e., we define $\Delta \phi_{q}=\phi_{q}-\phi_{q+1}$. As a special case $\Delta \phi_{k^{*}}$ is defined as $\Delta \phi_{k^{*}}=2 \pi+\phi_{1}-\phi_{k^{*}}$. Of course, $\sum_{l} \Delta \phi_{l}=2 \pi$. See figure 7.2 for an illustration.

We now establish an inequality that will lead us directly to the solution of our problem.

Lemma 7.2 Assume that $k^{*} \geq 3$. For any $p, 1 \leq p \leq k^{*}$, if $\Delta \phi_{p}>\pi$, then there is at least one vector $\mathbf{n}$ which is zero-covered by the given set of $k^{*}$ lights.

Proof For any two consecutive lights $i_{p}$ and $i_{p+1}$, we rotate the $x-y$ axis of our reference frame about the $z$-axis such that the positive $x$-axis bisects the azimuth angle between the two lights. In this frame, we have

$$
\mathbf{i}_{p}=\left(\begin{array}{c}
\sin \alpha \cos \phi \\
-\sin \alpha \sin \phi \\
\cos \alpha
\end{array}\right) \quad \mathbf{i}_{p+1}=\left(\begin{array}{c}
\sin \alpha \cos \phi \\
\sin \alpha \sin \phi \\
\cos \alpha
\end{array}\right)
$$

where, $\alpha$ is the zenith angle as before, and $\phi=\Delta \phi_{p} / 2$ is strictly greater than $\pi / 2$. (i.e. $\cos \phi<0$ ).

Now consider any vector $\mathbf{u}$, given by $\mathbf{u}=\left(\begin{array}{lll}1 & 0 & \zeta\end{array}\right)^{T}$, where,

$$
0<\zeta<-\tan \alpha \cos \phi .
$$

We can construct $\mathbf{n}$ such that $\mathbf{n}=\mathbf{u} /\|\mathbf{u}\|$. We have,

$$
\begin{aligned}
\mathbf{n}^{T_{\mathbf{i}_{p+1}}} & =\frac{1}{\|\mathbf{u}\|}[\sin \alpha \cos \phi+\zeta \cos \alpha] \\
& =\frac{1}{\|\mathrm{u}\|}\left[\sin \alpha \cos \phi\left(1+\frac{\zeta}{\tan \alpha \cos \phi}\right)\right]
\end{aligned}
$$

It is easy to show that the term $1+\frac{\zeta}{\tan \alpha \cos \phi}$ in the above expression is positive. However, $\cos \phi$ is negative. Hence, $\mathbf{i}_{p+1}^{T} \mathbf{n}<0$. Using the exact same argument, it is possible to show that $\mathbf{i}_{p}^{T} \mathrm{n}<0$. 


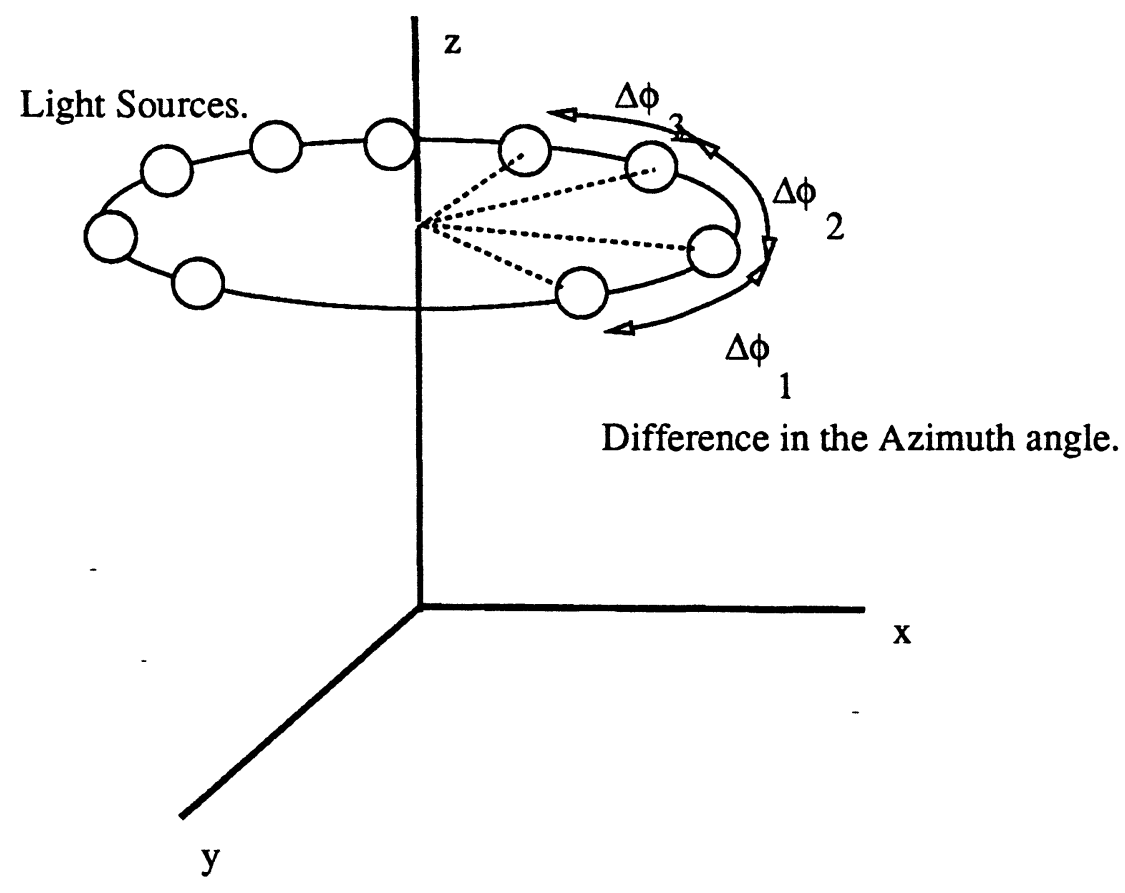

Figure 7.2 Configuration for Complete Reconstruction.

If we select any other light source $\mathbf{i}_{q}$, then we know that its zenith angle is $\alpha$ and that its azimuth angle $\phi_{q}$ is constrained by $2 \pi-\phi<\phi_{q}<\phi$. Since, $-\cos \phi<$ $-\cos \phi_{q}$ we get $-\tan \alpha \cos \phi<-\tan \alpha \cos \phi_{q}$. This leads us to $1+\frac{\zeta}{\tan \alpha \cos \phi_{q}}>0$ as before. Also, $\cos \phi_{q}<0$ as before, and using the same argument as above, we get $\mathbf{i}_{q}^{T} \mathbf{n}<0$.

Hence, any vector $\mathbf{n}$ of the form $\mathbf{u} /\|\mathbf{u}\|$ is zero-illuminated.

We will now proceed to establish the exact value of $k^{*}$.

Theorem 7.1 $k^{*}=2 k$ lights are necessary and sufficient to $k$ illuminate all vectors $\mathbf{n}$ that have a zenith angle strictly less than $\pi / 2$. These lights can be placed at an constant zenith angle $\alpha, 0<\alpha<\pi / 2$. The differences in the azimuth angles $\Delta \phi_{j}, j=1, \ldots, k^{*}$ have to be constrained such that $\Delta \phi_{j} \leq \pi$.

Proof The constraint on $\Delta \phi_{j}$ follows from a straight application of Lemma 7.2 to every consecutive pair of i's. 
However, applying Lemma 7.2 to pairs of i's is not sufficient. A more global constraint is required. This can be obtained as follows. Consider, the light sources $i_{2}, i_{3}, \cdots, i_{k}$. If we remove all of these lights, we have the lights $i_{k+1}$, $\mathbf{i}_{k+2}, \cdots, \mathbf{i}_{k^{*}}, \mathbf{i}_{1}$ left. We now show by contradiction that the difference between the azimuth angle between $i_{1}$ and $i_{k+1}$ must be less than or equal to $\pi$.

If this were not so, by application of Lemma 7.2 we see that there will exist vectors $\mathbf{n}$ that are zero-illuminated by these light sources. Re-introducing the lights $\mathbf{i}_{2}, \cdots, \mathbf{i}_{k}$ can, at the most, $k-1$ illuminate the $\mathbf{n}$ vectors, and we will not have a $k$-illumination. Hence, the difference between the azimuth angles between $i_{1}$ and $i_{k+1}$ must be less than or equal to $\pi$. This is, $\Delta \phi_{1}+\Delta \phi_{2}+\cdots+$ $\Delta \phi_{k} \leq \pi$.

Similar inequalities can be written by starting with $\mathbf{i}_{3}, \mathbf{i}_{4}, \cdots$ instead of $\mathbf{i}_{2}$. This leads us to the following:

$$
\begin{aligned}
\Delta \phi_{2}+\Delta \phi_{3}+\cdots+\Delta \phi_{k+1} & \leq \pi \\
\Delta \phi_{3}+\Delta \phi_{4}+\cdots+\Delta \phi_{k+2} & \leq \pi \\
\vdots & \\
\Delta \phi_{k^{*}}+\Delta \phi_{1}+\cdots+\Delta \phi_{k-1} & \leq \pi .
\end{aligned}
$$

Note that we have a net of $k^{*}$ inequalities, and that any particular term $\Delta \phi_{j}$ occurs in exactly $k$ of them. Adding all the inequalities, we get: $k \sum_{j} \Delta \phi_{j} \leq k^{*} \pi$. But, we know that $\sum_{j} \Delta \phi_{j}=2 \pi$. This leads us to $k^{*} \geq 2 k$, which establishes the necessary part of the theorem.

We now establish that $k^{*}=2 k$ lights are sufficient to $k$-illuminate all $\mathbf{n}$. Let the light sources $\mathbf{i}_{j}, j=1, \cdots, 2 k$ have the same zenith angle $\alpha, 0<\alpha<\pi / 2$; and be uniformly placed in their azimuth angle, i.e., the azimuth angle of $\mathbf{i}_{j}$ is $\phi_{j}=\frac{2 \pi}{2 k}(j-1)$. Given any $\mathbf{n}$ and $\mathbf{i}_{j}$ we consider their projections $\mathbf{n}_{x y}$ and $\mathbf{i}_{x y, j}$ on the $x-y$ plane. If

$$
\mathbf{n}=\left(\begin{array}{c}
\cos \phi^{\prime} \sin \theta^{\prime} \\
\sin \phi^{\prime} \sin \theta^{\prime} \\
\cos \theta^{\prime}
\end{array}\right) \quad \mathbf{i}_{j}=\left(\begin{array}{c}
\cos \phi_{j} \sin \alpha \\
\sin \phi_{j} \sin \alpha \\
\cos \alpha
\end{array}\right)
$$

then,

$$
\mathbf{n}_{x y}=\left(\begin{array}{c}
\cos \phi^{\prime} \sin \theta^{\prime} \\
\sin \phi^{\prime} \sin \theta^{\prime}
\end{array}\right) \quad \mathbf{i}_{x y, j}=\left(\begin{array}{c}
\cos \phi_{j} \sin \alpha \\
\sin \phi_{j} \sin \alpha
\end{array}\right)
$$


Note that since $\cos \alpha, \cos \theta^{\prime}>0, \mathbf{i}_{x y, j}^{T} \mathbf{n}_{x y} \geq 0$ implies $\mathbf{i}_{j}^{T} \mathbf{n}>0$. The tips of the vectors $\mathbf{i}_{x y, j}$ are uniformly distributed in a circle around the origin of the $\mathrm{x}-\mathrm{y}$ plane with an angle of $\frac{2 \pi}{2 k}$ between any two consecutive vectors. It is easy to see that given any vector $\mathbf{n}_{x y}$ in this plane, there are at least $k$ vectors (amongst $\mathbf{i}_{x y, j}$ ) that fall within $\pm \pi / 2$ of $\mathbf{n}_{x y}$. Hence, there are at least $k$ vectors $\mathbf{i}_{x y, j}$ such that $\mathbf{i}_{x y, j}^{T} \mathbf{n}_{x y} \geq 0$. It thereby follows that there are at least $k$ vectors $\mathbf{i}_{j}$ such that $\mathbf{i}_{j}^{T} \mathbf{n}>0$.

Note that from the later half of the proof it follows that no three of the light sources are linearly dependent.

\subsection{Discussion}

The considerations of this chapter merit some discussion. Adding extra light sources is a feasible way to reconstruct a surface completely. However, there are limitations to the number of light sources that can be realistically added in any photometric stereo system. Adding extra light sources forces neighboring light source directions to be more aligned. Thus the variation in image intensity due to alternate switching of lights is reduced and the reconstruction is in the danger of being sensitive to noise in the data. 


\section{Chapter 8}

\section{Conclusions}

In conclusion, then, we have established several facts. Perhaps the most central result to emerge from this work is that diffuse reflection from real-world surfaces can be significantly non-Lambertian and that this non-Lambertian nature has to be taken into account for a good reconstruction. Diffuse reflection has a lobe structure and we have been able to analyze the origin of the lobes from the physics of light scattering. The lobe-like structure has an especially pleasing mathematical form for phase angles less that $30^{\circ}$. We were able to exploit this structure in this work. The assumption about the phase angle is the most restrictive of all the assumptions made here and while most photometric stereo experiments do fall close to this range, it would be nice to extend the theory to wider phase angles. To do this, we will have to explicitly take the shadowing function and the Fresnel reflectivities of equation (3.11) into account instead of approximating them as unity. An expression for both of them which is simpler than equation (3.11) would go a long way towards achieving this, but currently such an expression is unavailable. An empirical fit of some "nice" function to the exact expression seems like the best way to proceed, but such a fit would have to be experimentally justified for a large class of surfaces. At the present, this falls out of the scope of current work.

On the other hand, as we saw in chapters 3 and 4 the reflectance maps proposed here give about an order of magnitude increased accuracy in modeling and reconstructing real-world surfaces when compared with classical techniques. Also, we were able to establish several theoretical results which put the technique on a firmer foundation. In particular, the establishment of the number of light sources needed to obtain unique solutions is a useful result. In all of the previous work, either significant sections of the work were devoted to analytic proofs of uniqueness for explicit forms of reflectance maps, or failing this, to qualitative and graphical "proofs" for special cases. We have been able to es- 
tablish uniqueness for entire families of reflectance maps and demonstrate that the theoretical results do yield useful reconstructions in practice.

For un-normalized photometric stereo, we were able to formulate a framework within which questions about the problem could be posed as questions about the geometry of a manifold. We were able to establish several ground results using this framework. Currently, our inability to express the manifold implicitly holds us back from investigating this approach further. It is likely that an implicit expression for the manifold will be fairly complex. (The implicit expression for a bi-cubic surface, for example, is an $18^{\text {th }}$ degree polynomial in $x, y$ and $z$ having over a thousand terms.) Again, some simple empirical formulation of the implicit form which would work for the physical reflectance map of equation (3.19) would be useful and will certainly be the goal of future research.

This frame work also provides a basis to reason about other aspects of photometric stereo. About the effect of noise, for example. The solution to the problem of normalized photometric stereo with noise can be seen to be the problem of projecting a data point on to the manifold. In the Lambertian case, the manifold is an ellipsoid and hence convex. A unique solution can therefore be obtained for the noisy case. For a non-Lambertian reflectance map, the manifold is no longer convex and any single data point could generate multiple solutions. Also if the manifold is appropriately shaped, small changes in data point could cause large changes in the solutions, once again raising the possibility of ill-posedness. Clearly, this is a subject for future study too.

Besides the results we obtained, the fact that we can reason about a large number of previously unaddressed issues within our framework demonstrates the utility of our approach.

We were also able to formulate the problem of joint estimation. As we saw in chapter 6 this problem is ill-posed and has to be regularized. However, the extent of regularization used in this work was not excessive and we were able to get reasonable reconstructions using it.

Finally, we looked at the geometric problem of completeness of reconstruction and were able to establish that we need twice the number of light sources for a complete reconstruction as we need for a unique reconstruction. 


\section{Appendix A}

\section{Surface Normal to Depth Conversion.}

In this appendix we will state the details of the algorithm that converts the surface normals into depth information. From the surface normal $\mathbf{n}(i, j)$ at any point, the surface slopes $p(i, j)=\frac{d z(i, j)}{d x}$ and $q(i, j)=\frac{d z(i, j)}{d y}$ are calculated as follows:

$$
\text { If } \mathbf{n}(i, j)=\left(\begin{array}{l}
\mathbf{n}_{x}(i, j) \\
\mathbf{n}_{y}(i, j) \\
\mathbf{n}_{z}(i, j)
\end{array}\right)
$$

then,

$$
\begin{aligned}
& p(i, j)=-\frac{\mathbf{n}_{x}(i, j)}{\mathbf{n}_{z}(i, j)}, \\
& q(i, j)=-\frac{\mathbf{n}_{y}(i, j)}{\mathbf{n}_{z}(i, j)} .
\end{aligned}
$$

The algorithm proposed by Silver [47] was used to calculate the depth from $p$ and $q$. Silver's algorithm assumes the depth at some point $i_{o}, j_{o}$ and iteratively calculates the depth at every other point on the surface according to:

$$
\begin{aligned}
z\left(i_{o}, j_{o}\right)= & 100, \\
z\left(i \pm 1, j_{o}\right)= & z\left(i, j_{o}\right) \pm \frac{p\left(i, j_{o}\right)+q\left(i \pm 1, j_{o}\right)}{2}, \\
z\left(i_{o}, j \pm 1\right)= & z\left(i_{o}, j\right) \pm \frac{p\left(i_{o}, j\right)+q\left(i_{o}, j \pm 1\right)}{2}, \\
z(i \pm 1, j+1)= & \frac{1}{2}\left(z(i, j+1) \pm \frac{p(i, j+1)+p(i \pm 1, j+1)}{2}\right) \\
& +\frac{1}{2}\left(z(i \pm 1, j)+\frac{q(i \pm 1, j)+q(i \pm 1, j+1)}{2}\right), \\
z(i \pm 1, j-1)= & \frac{1}{2}\left(z(i, j-1) \pm \frac{p(i, j-1)+p(i \pm 1, j-1)}{2}\right) \\
& +\frac{1}{2}\left(z(i \pm 1, j)-\frac{q(i \pm 1, j)+q(i \pm 1, j-1)}{2}\right) .
\end{aligned}
$$




\section{Appendix B}

\section{Apparatus and Experimental Procedure}

This appendix contains details of the apparatus and the experimental procedure for the reconstructions of this thesis. The apparatus is shown in figure B. A bi-cycle wheel with a radius of about $1 \mathrm{ft}$ was mounted on a vertical stand which was firmly fixed to a table. The camera was mounted along the axis of the wheel. A light bulb was fixed on the rim of the wheel and served as the light source. The wheel could be rotated to move the bulb around the camera. When the apparatus was properly aligned and the object placed on the table under the camera, rotating the wheel caused the azimuth angle of the light source to change while keeping its zenith angle constant. The wheel assembly could be tilted to achieve vertical orientation of the camera axis. An indicator along with a protractor was also fixed on the wheel. Together they provided a direct read-off of the azimuth angle of the light source.

The apparatus was calibrated in the following way

(a) The light bulb was chosen with some care. After working with a number of bulbs it was discovered that there was significant undesirable variation in the amount of irradiation on the object as the azimuth angle of the bulb was changed. The structure of the bulb filament appeared to be the cause of of this variation. After trying out different bulbs, an ordinary 40 watt clear bulb was chosen for the rest of the experimentation. Over a change in the azimuth angle of $360^{\circ}$ it was observed that the variation of incident irradiation over the area of the object was about $5 \%$. (The irradiation was measured with an Ealing Optics Photometer model 29074.)

(b) Next, the camera response was calibrated using the photometer. Under the condition of pitch darkness, the camera yielded a grey level value of 33. This value was subtracted from all subsequent grey level values. It was then found that the response of the camera, when calibrated against the 


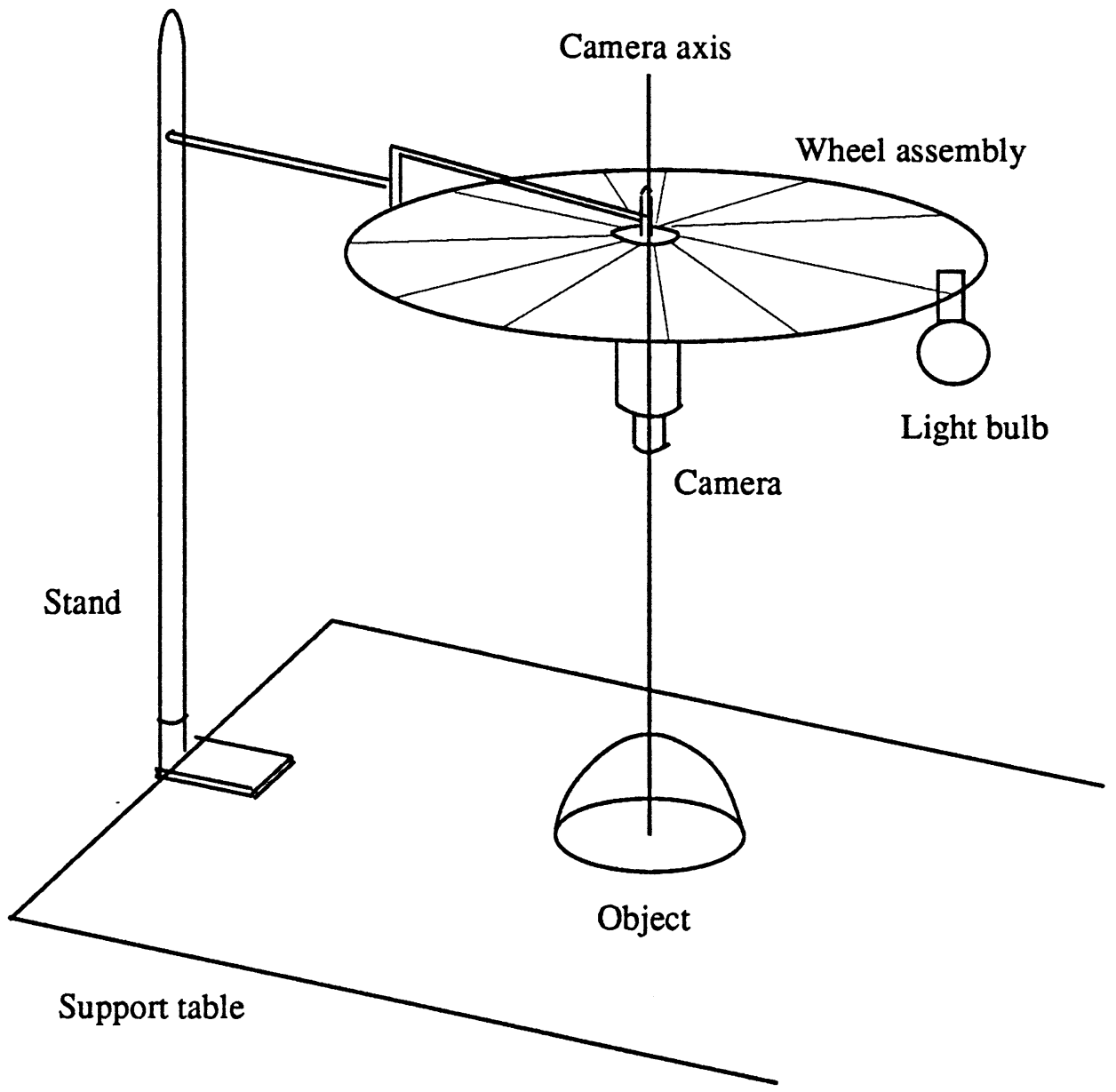

Figure B.1 The Experimental Apparatus. 
response of the photometer, was linear with a maximum deviation from linearity of about $2 \%$ of its full scale response (the full scale response being $255-33=223$ ). The camera was assumed to be linear from this point onwards.

(c) Next, the geometry of the apparatus was calibrated. A known Lambertian sphere was imaged with the light bulb and the intensity in the principal plane of the image was obtained. A model of a Lambertian surface illuminated at a variable incidence angle was fit to these set of intensities. The incidence angle at which the best fit was obtained was assumed to be the actual angle of incidence. The vertical height and the tilt of the bi-cycle wheel was adjusted such that the best fit incidence angle over the range of azimuth angles of interest was $25^{\circ}$ to within $\pm 2^{\circ}$. The vertical distance from the support table to the center of the wheel was about $3 \mathrm{ft}$.

After calibration, the reconstruction experiments were conducted in a straight forward manner by positioning the light source at any azimuth angle and obtaining the image. For every position of the light source, 20 images were obtained and averaged to reduce noise. The value of 33 was subtracted as before from every grey level and the resulting images were used in the reconstruction. 


\section{Bibliography}

[1] H. P. Baltes. Inverse Source Problems in Optics. Springer-Verlag, 1978.

[2] H. P. Baltes. Inverse Scattering Problems in Optics. Springer-Verlag, 1980.

[3] S. T. Barnard. Stochastic stereo matching over scale. In Proc. DARPA Image Understanding Workshop, pages 769-778, 1988.

[4] L. P. Bayvel and A. R. Jones. Electromagnetic Scattering and its Applications. Applied Science Publishers, 1981.

[5] P. Beckmann. Shadowing of random rough surfaces. I.E.E.E. Trans. Ant. and Propagation, AP-13:384-388, May 1965.

[6] P. Beckmann and A. Spizzichino. The Scattering of Electromagnetic Waves from Rough Surfaces. Pergamon Press, 1963.

[7] S. Bertram. The unamace and the automatic photomapper. Photogrammetric Engineering, 35(6):569-576, June 1969.

[8] R. C. Birkebak and E. R. G. Eckert. Effect of roughness of metal surfaces on angular distribution of monochromatic reflected light. Journal of Heat Transfer, pages 85-94, February 1965.

[9] K. L. Boyer and A. C. Kak. Structural stereopsis for 3-d vision. I.E.E.E. Trans. P.A.M.I., 10(2):144-166, March 1988.

[10] W. M. Brandenberg and J. T. Neu. Unidirectional reflectance of imperfectly diffuse surfaces. J. Opt. Soc. Amer., 56(1):97-103, January 1966.

[11] M. Q. Brewster and C. L. Tien. Radiative transfer in packed fluidized beds: Dependent versus independent scattering. Journal of Heat Transfer, 104:573-579, November 1982.

[12] R. A. Brockelman and T. Hagfors. Note on the effect of shadowing on the backscattering of waves from a random rough surface. I.E.E.E. Trans. Ant. and Propagation, AP-14:621-626, September 1966. 
[13] J. D. Cartigny, Y. Yamada, and C. L. Tien. Radiative transfer with depedent scattering by particles: Part 1 - theoretical investigation. Journal of Heat Transfer, 108:608-613, August 1986.

[14] S. Chandrasekhar. Radiative Transfer. Dover Publications, Inc, 1960.

[15] E. N. Coleman and R. Jain. Obtaining 3-dimensional shape of textured and specular surfaces using four-source photometry. C.G.I.P., 18:309-328, April 1982.

[16] H. Cramer and M. R. Leadbetter. Stationary and Related Processes. John Wiley and Sons, 1967.

[17] A. I. Funai and R. E. Rolling. Inspection techniques for the characterization of smooth, rough, and oxidized surfaces. In G. B. Heller, editor, Thermophysics of Spacecraft and Planetary Bodies. Academic Press, 1967.

[18] G. Hardy, J. E. Littlewood, and G. Polya. Inequalities. Cambridge University Press, 1934.

[19] G. Healy and T. O. Binford. Local shape from specularity. In First Intl. Conf. on Computer Vision, pages 151-160, June 1987.

[20] B. J. Hoenders, E. Jakeman, H. P. Baltes, and B. Steinle. K correlations and facet models in diffuse scattering. Opta. Acta., 26:1307-1319.

[21] B. K. P. Horn. Understanding image intensities. Artificial Intelligence, 8:201-231, 1977.

[22] B. K. P. Horn. Robot Vision. M.I.T. Press, 1986.

[23] B. K. P. Horn and R. W. Sjoberg. Calculating the reflectance map. Applied Optics, 18(11):1770-1779, June 1979.

[24] H. C. Hottel, A. F. Sarofim, W. H. Dalzell, and I. A. Vasalos. Multiple scatter: Comparison of theory with experiment. Journal of Heat Transfer, pages 285-291, May 1970.

[25] H. C. Hottel, A. F. Sarofim, W. H. Dalzell, and I. A. Vasalos. Optical properties of coatings. effect of pigment concentration. AIAA Journal, 9(10):1895-1898, October 1971.

[26] A. F. Houchens and R. G. Hering. Bi-directional reflectance of rough metal surfaces. In G. B. Heller, editor, Thermophysics of Spacecraft and Planetary Bodies. Academic Press, 1967. 
[27] K. Ikeuchi. Determining surface orientations of specular surfaces by using the photometric stereo method. I.E.E.E. Trans. P.A.M.I., 3(6):661-669, November 1981.

[28] K. Ikeuchi. Shape from regular patterns. Artificial Intelligence, 22(1):4975, January 1984.

[29] K. Ikeuchi and B. K. P. Horn. Picking up an object from a pile of objects. In R. Paul, editor, Robotics Research: The First International Seminar. M.I.T. Press, 1984.

[30] IMSL. IMSL Math Library. Houston,TX.

[31] E. Jakeman and P. N. Pusey. Non-gaussian fluctuations in electromagnetic radiation scattered by a random phase screen. Jour. Phys. A., 8:369-391, 1975 .

[32] F. A. Jenkins and H. E. White. Fundamentals of Optics. McGraw-Hill, Inc., 1957.

[33] D. H. Johnson. Personal Notes.

[34] M. Kerker. The Scattering of Light and Other Electromagnetic Radiation. Academic Press, 1969.

[35] G. Kortum. Reflectance Spectroscopy. Springer-Verlag, 1969.

[36] J. E. Loehrlein, E. R. F. Winter, and R. Viskanta. Measurement of bidirectional reflectance using a photometric technique. In G. B. Heller, editor, Thermophysics of Spacecraft and Planetary Bodies. Academic Press, 1967.

[37] T. J. Love and R. E. Francis. Expermental determination of reflectance function for type 302 stainless steel. In G. B. Heller, editor, Thermophysics of Spacecraft and Planetary Bodies. Academic Press, 1967.

[38] D. Marr. Vision. W. H. Freeman and Co., 1982.

[39] S. K. Nayar, K. Ikeuchi, and T. Kanade. Extracting shape and reflectance of lambertian, specular and hybrid surfaces. Technical Report CMU-RITR-88-14, C.M.U., August 1988.

[40] F. E. Nicodemus, J. C. Richmond, J. J. Hsia, I. W. Ginsberg, and T. Limperis. Geometrical Considerations and Nomenclature for Reflectance. National Bureau of Standards, 1977. 
[41] C. Pask. Derivation of source-field coherence properties from radiation angular distribution. Opta. Acta., pages 235-240, 1977.

[42] R. Ray, J. Birk, and R. Kelly. Error analysis of surface normals determined by radiometry. I.E.E.E. Trans. P.A.M.I., PAMI-5(6), November 1983.

[43] W. A. Rense. Polarization studies of light diffusely reflected from ground and etched glass surfaces. J. Opt. Soc. Amer., 40(1):55-59, January 1950.

[44] S. O. Rice. Reflection of electromagnetic waves from slightly rough surfaces. Comm. Pure Appl. Math., pages 351-378, 1951.

[45] T. W. Sederberg. Implicit and Parametric Curves and Surfaces for Computer Aided Geometric Design. PhD thesis, Purdue, 1983.

[46] R. Siegel and J. R. Howell. Thermal Radiation Heat Transfer. McGrawHill, 1972.

[47] W. M. Silver. Determining shape and reflectance using muliple images. Master's thesis, MIT, 1980.

[48] B. G. Smith. Geometrical shadowing of a random rough surface. I.E.E.E. Trans. Ant. and Propagation, AP-15(5):668-671, Sept 1967.

[49] K. A. Stevens. The visual interpretation of surface contours. Artificial Intelligence, 17(1-3):47-73, August 1981.

[50] J. M. Stone. Radiation and Optics. McGraw-Hill Book Company, Inc., 1963.

[51] A. N. Tikhonov and V. A. Arsenin. Solutions of Ill-posed Problems. John Wiley and Sons, 1977.

[52] K. E. Torrance and E. M. Sparrow. Biangular reflectance of an electric non-conductor as a function of wavelength and surface roughness. Journal of Heat Transfer, pages 283-292, May 1965.

[53] K. E. Torrance and E. M. Sparrow. Off-specular peaks in the directional distribution of reflected thermal radiation. Journal of Heat Transfer, pages 223-230, May 1966.

[54] K. E. Torrance and E. M. Sparrow. Theory of off-specular reflection from roughened surfaces. J. Opt. Soc. Amer., 57(9):1105-1114, September 1967. 
[55] K. E. Torrance, E. M. Sparrow, and R. C. Birkebak. Polarization, directional distribution, and off-specular peak phenomenon in light reflected from roughened surfaces. J. Opt. Soc. Amer., pages 916-925, July 1966.

[56] H. C. van de Hulst. Light Scattering by Small Particles. Dover Publications, Inc., 1957.

[57] F. Varnier, M. Rasigni, J. P. Palmari, and A. Llebaria. Height and slope distributions for surfaces of rough mettalic deposits. Applied Optics, 21(20):3681-3684, October 1982.

[58] R. J. Wagner. Shadowing of randomly rough surfaces. J. Acoust. Soc. Amer., 41(1):138-151, 1967.

[59] L. B. Wolff. Spectral and polarization stereo methods using a single light source. In Proc. Image Understanding Workshop, volume 2, pages 810-820, 1987.

[60] R. J. Woodham. Reflectance map techniques for analyzing surface defects in metal castings. M.I.T. AI Lab Techincal Report 457, June 1978.

[61] Y. Yamada, J. D. Cartigny, and C. L. Tien. Radiative transfer with depedent scattering by particles: Part 2 - experimental investigation. Journal of Heat Transfer, 108:614-618, August 1986. 